$5-10-2021$

\title{
More than Brides Alliance (MTBA) : Rapport d'évaluation finale
}

\author{
Andrea J. Melnikas \\ Population Council \\ Grace Saul \\ Population Council \\ Michelle Chau \\ Neelanjana Pandey \\ Population Council \\ James Mkandawire
}

See next page for additional authors

Follow this and additional works at: https://knowledgecommons.popcouncil.org/departments_sbsr-pgy How does access to this work benefit you? Let us know!

\section{Recommended Citation}

Melnikas, Andrea J., Grace Saul, Michelle Chau, Neelanjana Pandey, James Mkandawire, Mouhamadou Gueye, Aissa Diarra, and Sajeda Amin. 2021. "More than Brides Alliance (MTBA) : Rapport d'évaluation finale." New York: Population Council. 


\section{Authors}

Andrea J. Melnikas, Grace Saul, Michelle Chau, Neelanjana Pandey, James Mkandawire, Mouhamadou Gueye, Aissa Diarra, and Sajeda Amin 


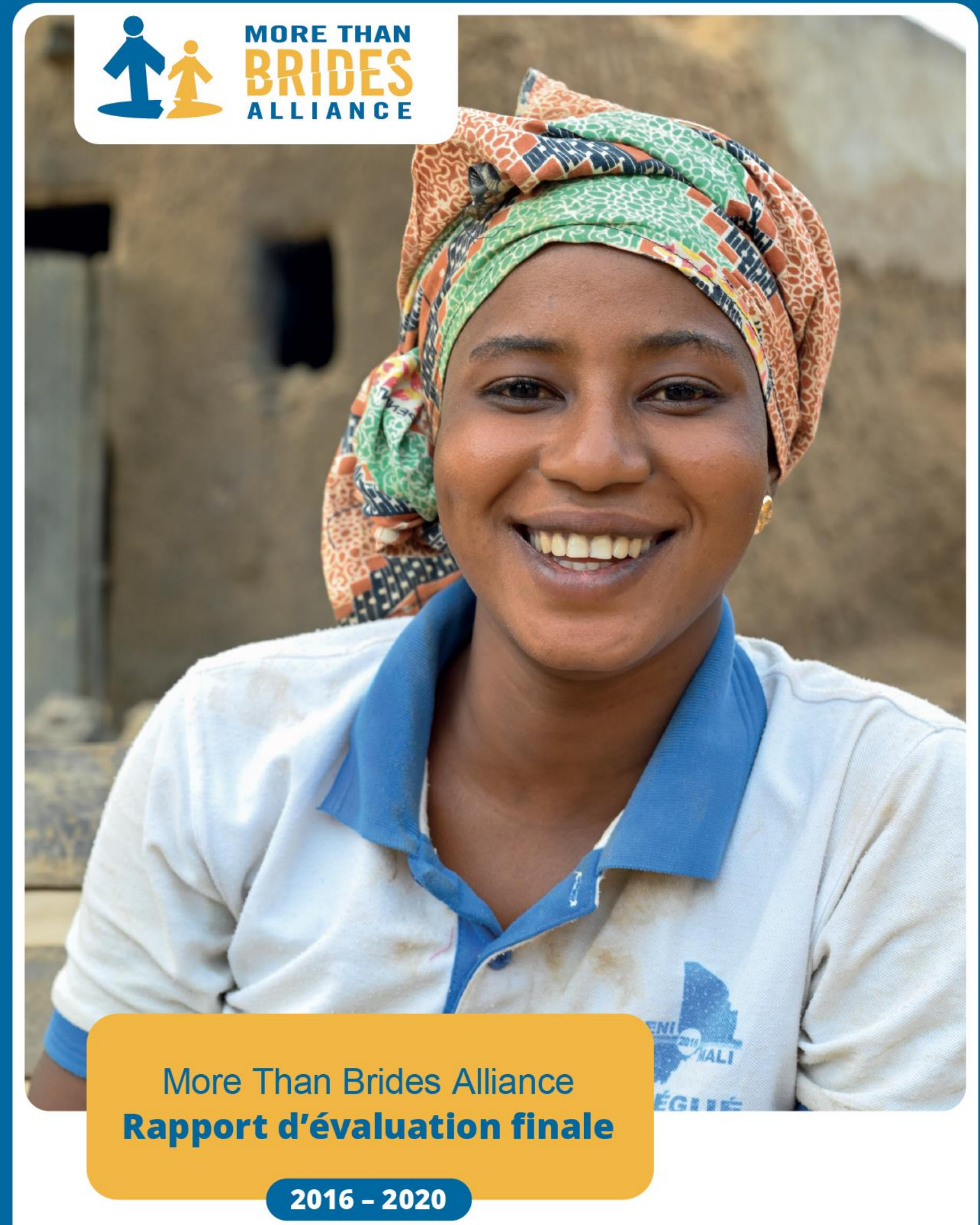

Inde - Malawi - Mali - Niger 
Population Council s'attelle aux questions cruciales de la santé et du développement - qu'il s'agisse d'endiguer la progression du VIH, d'améliorer la santé reproductive et d'offrir aux jeunes la promesse d'une vie satisfaisante et productive. Par ses travaux de recherche en biomédecine, en sciences sociales et en santé publique dans 50 pays, Population Council s'efforce d'apporter avec ses partenaires des solutions qui conduisent à des politiques, des programmes et des technologies plus efficaces, pour améliorer la vie dans le monde. Fondé en 1952, Population Council est une organisation non gouvernementale à but non lucratif qui siège à New York sous la conduite d'un conseil d'administration international.

\section{Population Council}

1 Dag Hammarskjold Plaza, $3^{\text {rd }}$ Floor

New York, NY 10017

États-Unis

212-339-0500

popcouncil.org

Citation suggérée: Melnikas AJ, Saul G, Chau M, Pandey, N, Mkandawire J, Gueye M, Diarra A, Amin S. 2021. "More than Brides Alliance (MTBA) : Rapport d'évaluation finale. » New York, Population Council.

Crédit photographique : Ousmane Samassekou/ Oxfam Novib

Graphiques : Mike Vosika

(c) 2021 The Population Council, Inc. 


\section{Table des matières}

Remerciements

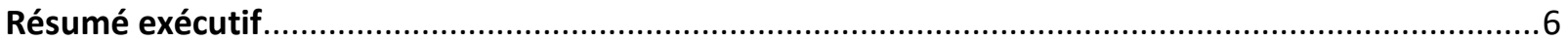

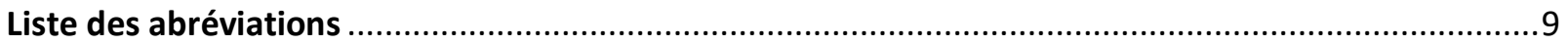

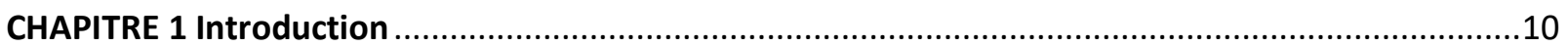

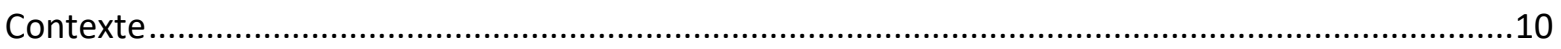

Partenaires du programme MNCP et contextes d'intervention .......................................................11

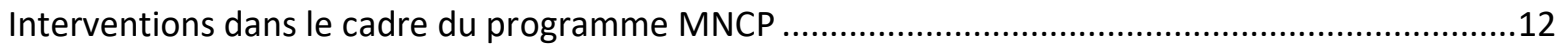

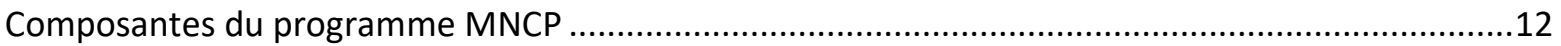

Adaptations de la programmation à la pandémie Covid-19..........................................................14

Conception et objectifs de la recherche du programme MNCP.....................................................14

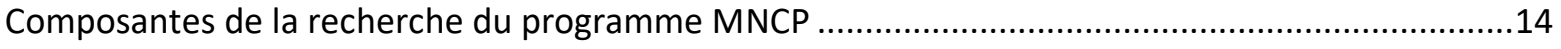

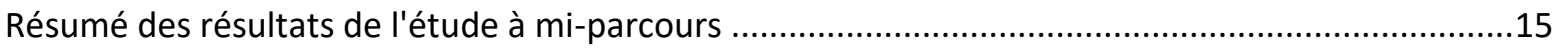

Objectifs de l'évaluation finale et adaptations liées à la Covid-19 ..............................................15

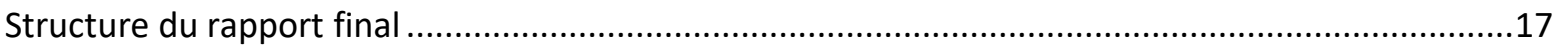

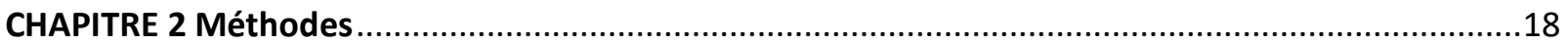

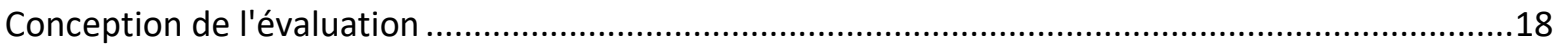

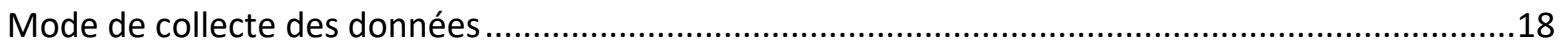

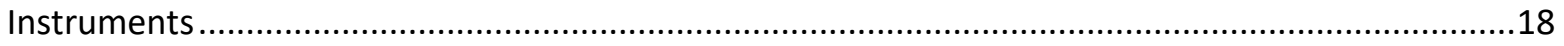

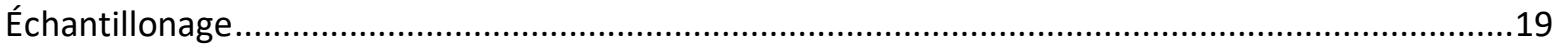

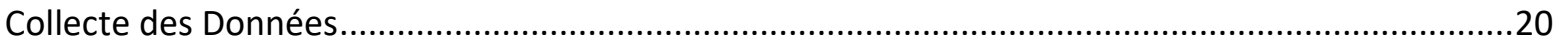

Revue éthique

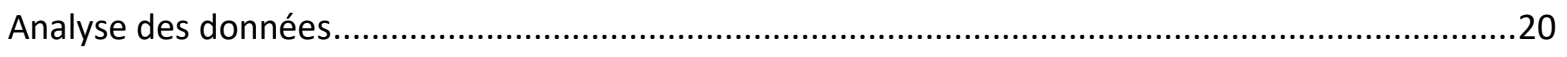

CHAPITRE 3 Impact de la pandémie de Covid-19 sur les adolescentes .............................................22

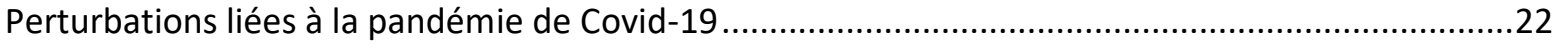

Impact de la Covid-19 sur le mariage des enfants ........................................................................22

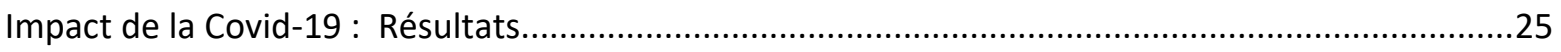

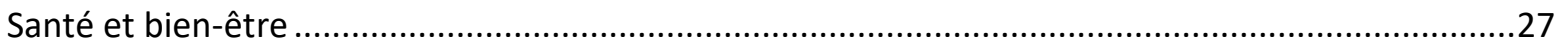

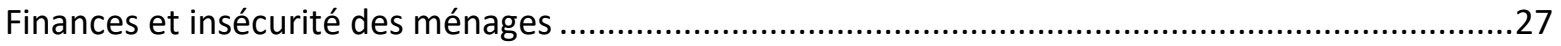

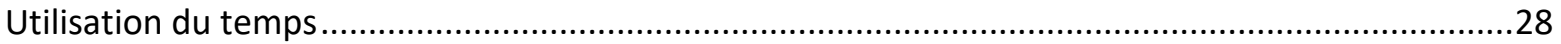

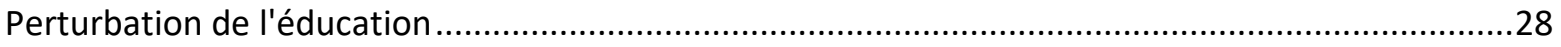

Implications pour l'interprétation des résultats de l'étude finale du MNCP ...................................28 
Contexte

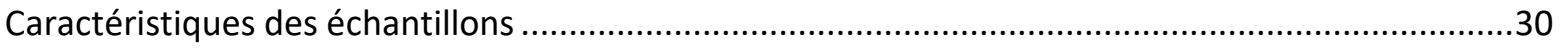

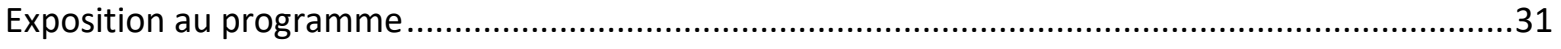

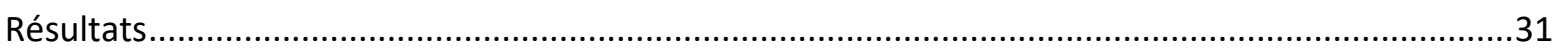

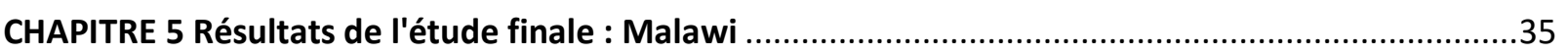

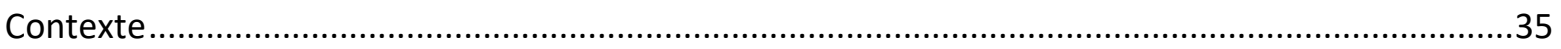

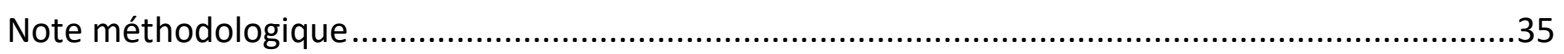

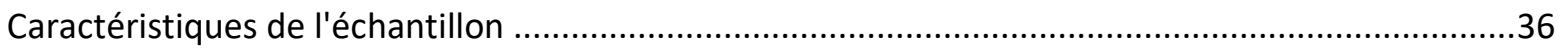

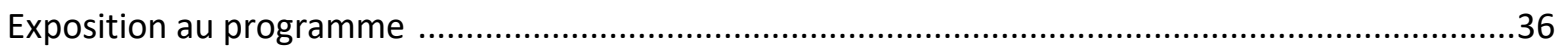

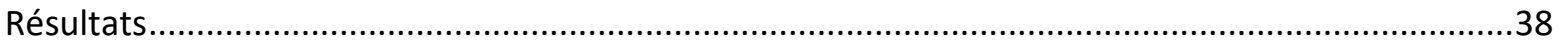

CHAPITRE 6 Résultats de l'étude finale : Mali ............................................................................

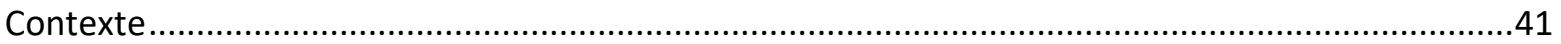

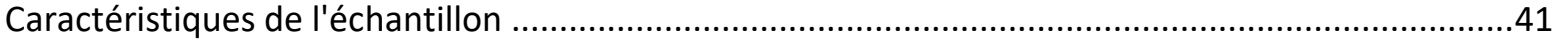

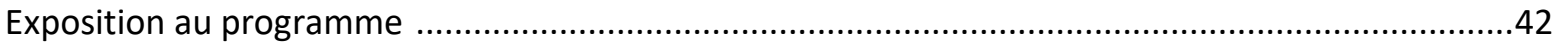

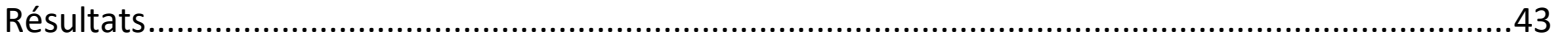

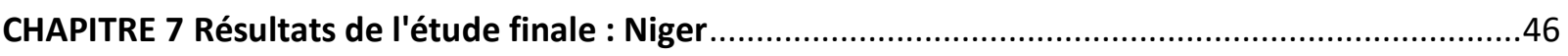

Contexte

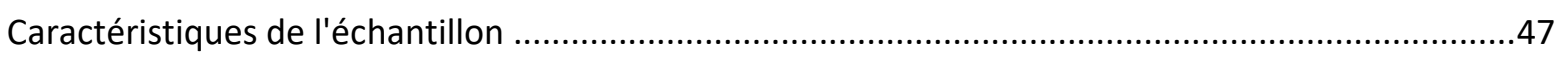

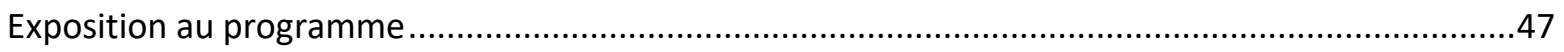

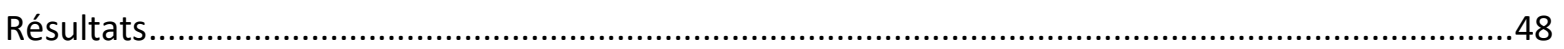

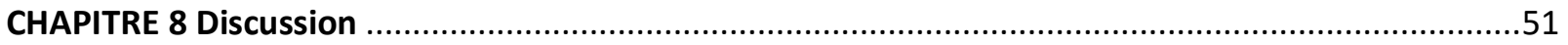

Les tendances du mariage : Étude de base-Étude à mi-parcours-Étude finale ............................51

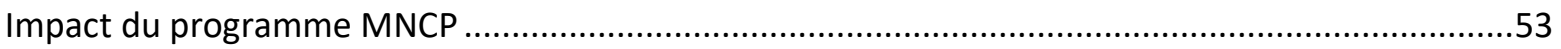

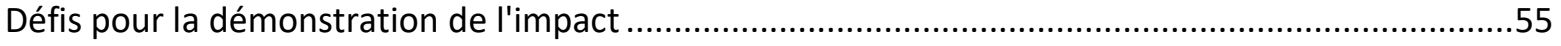

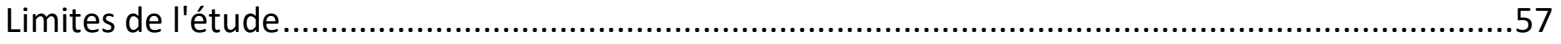

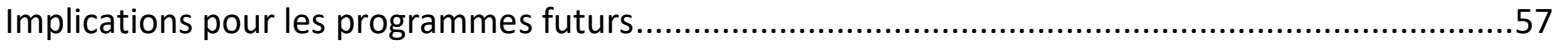

Annexe 1: Partenaires de la mise en œuvre par pays et état/ région .................................................59

Annexe 2: Différences de conception des études ...........................................................................60

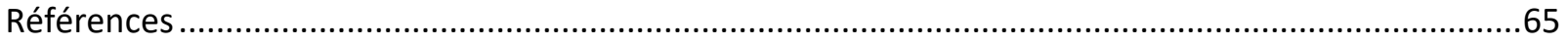




\section{Remerciements}

Un certain nombre de personnes ont contribué au rapport intermédiaire de l'Alliance «Plus qu'une épouse » et nous apprécions leurs efforts.

Nous remercions les membres de l'Alliance "More than Brides », notamment Save the Children PaysBas, Oxfam Novib, et Simavi pour leurs contributions et leur collaboration sur ce projet.

Nous remercions Mike Vosika pour son appui en matière de graphisme et de mise en page du rapport final. Nous remercions Joyce Altman pour son soutien rédactionnel.

Nous remercions également le Ministère des Affaires étrangères des Pays-Bas pour son appui à l'Alliance «More than Brides » et au volet recherche et évaluation en particulier.

Nous avons eu la chance de travailler avec des équipes de recherche talentueuses qui ont joué un rôle déterminant dans la réalisation du recrutement des ménages et des enquêtes auprès des adolescentes et des jeunes femmes. Auprès de Invest in Knowledge Initiative (IKI) au Malawi, nous remercions nos collègues Hamza Daud, Sydney Rodney Lungu, et Nancy Mulauzi, qui ont supervisé la collecte des données sur le terrain. Au CERIPS au Mali, nous remercions Sékou Omar Fofana, Timbaleck Traoré, et Ismalia Konaté et au LASDEL au Niger, nous remercions Chaibou Saadou, Issaley Nana Aichato, Ali Bako et Siddou Moumouni.

Enfin, nous remercions les participants à la recherche, en particulier les adolescentes et les jeunes femmes qui ont partagé leurs expériences, leurs pensées et leurs ambitions avec l'équipe de recherche. Nous les remercions de leur participation, sans laquelle cette étude n'aurait pas été possible. 


\section{Résumé exécutif}

Ce rapport final couvre le projet de l'Alliance More than Brides (MTBA) « Le mariage n'est pas un jeu d'enfant » (Marriage: No Child's Play - MNCP) mis en œuvre dans quatre pays : Inde, Malawi, Mali et Niger. Le projet MNCP vise à renforcer l'autonomie des filles, à les sensibiliser aux risques du mariage précoce, à améliorer leur accès aux services de santé sexuelle et reproductive et à soutenir les normes sociales favorables à l'éducation des filles, à leur engagement économique et à leur participation aux décisions conjugales. La MTBA est composée de partenaires tels que Save the Children Pays-Bas, Simavi, Oxfam-Novib et le Population Council, et de 25 partenaires locaux.

Ce rapport présente les résultats de l'évaluation du projet MNCP, notamment les résultats de la dernière année, caractérisée par des adaptations majeures, tant au niveau programmatique que de la recherche, liées à la crise de la Covid-19. D'une manière générale, le programme MNCP vise à fournir des estimations sur l'impact du programme et ses tendances de manière comparable dans des contextes qui diffèrent en termes de prévalence et de facteurs favorisant la prévalence du mariage des enfants. Le rapport explore des questions telles que le mariage des enfants, la scolarisation, le travail et la grossesse ainsi que les indicateurs de connaissances et d'attitudes basés sur des études transversales menées auprès d'adolescentes âgées de 12 à 19 ans dans les villages d'intervention et de comparaison à trois tours $(2016 / 7,2018,2020)$ et des enquêtes menées auprès des parents d'adolescentes ou des membres adultes de leurs familles (2020).

Ce rapport décrit l'impact, tel que mesuré par les résultats de l'analyse de l'écart dans les différences (EDD) en utilisant les études de base et finales, en comparant le changement dans les zones d'intervention par rapport au changement dans les zones de comparaison. Les analyses ont été effectuées dans Stata SE 14.2 et ont inclus des ajustements basés sur la conception de l'évaluation et la fidélité à la randomisation.

Nous constatons qu'en Inde, le programme MNCP a eu un impact significatif sur plusieurs indicateurs clés. La proportion de filles mariées a davantage diminué dans les zones d'intervention que dans les zones de comparaison dans l'échantillon combiné des quatre états indiens, ainsi que dans 3 des 4 états pris individuellement. Bien que le mariage des enfants ait diminué dans les zones de comparaison au cours de la même période, les baisses dans les zones d'intervention étaient plus importantes et statistiquement significatives $(p<0,01)$. La prévalence du mariage des enfants dans les zones d'intervention a diminué de $14,5 \%$ à 4,5\%, soit une baisse de $69 \%$ (alors que dans les zones de comparaison, la prévalence du mariage des enfants a diminué de $22 \%(p<0,05)$ ). Au Malawi, au Mali et au Niger, nous n'avons constaté aucun impact du programme sur la proportion de filles actuellement mariées ; dans chacun de ces pays, le mariage des enfants a diminué, mais il a diminué aussi bien dans les zones d'intervention que dans les zones de comparaison de sorte que l'impact ne peut pas être attribué au programme MNCP.

La connaissance du mariage des enfants, notamment la connaissance de l'âge légal du mariage et la capacité à citer les effets négatifs du mariage des enfants, s'est améliorée chez les adolescentes dans la plupart des pays du programme MNCP. Un impact significatif a été constaté en Inde, au Malawi, et au Niger sur la connaissance de l'âge légal du mariage. En Inde 
et au Niger, un impact significatif a été constaté sur la connaissance des effets négatifs du mariage des enfants.

Le programme d'intervention MNCP a permis d'influencer avec succès les voies alternatives au mariage des enfants, notamment en augmentant les possibilités d'éducation et les moyens de subsistance des filles. Nous constatons des effets significatifs sur la scolarisation, le niveau d'études et le travail des filles, mais aucun schéma clair n'est apparu dans les différents pays. Au Niger, le programme MNCP a montré son impact en augmentant la proportion de filles travaillant pour un revenu entre l'étude de base et l'étude finale. Au Malawi, le programme a pu avoir un impact démontrable sur plusieurs indicateurs liés à l'éducation, notamment en augmentant la proportion de filles ayant déjà fréquenté l'école, en augmentant le nombre moyen d'années d'éducation accomplies et en diminuant le taux d'analphabétisme chez les filles âgées de 12 à 19 ans.

Nous avons également examiné comment la Covid-19 et les restrictions associées au contrôle de l'infection ont influencé la vie des jeunes filles. Une proportion importante de ménages a déclaré avoir connu des pénuries alimentaires depuis mars 2020 (62,7 à 81,0\% des ménages répondants ont déclaré des pénuries alimentaires dans les quatre pays). En outre, 78,0 à $85,5 \%$ des adultes interrogés dans les quatre pays ont déclaré que la Covid-19 avait eu un impact négatif sur les finances de leur ménage. Les filles ont également déclaré se sentir plus déprimées depuis le début de la pandémie (47,2 à $86,4 \%)$ et avoir de plus lourdes tâches de soins à la maison (37,5 à $58,6 \%$ ).

Dans le cadre de l'évaluation de l'impact du programme MNCP un certain nombre de limitations doivent être prises en compte. La pandémie de Covid-19 et les restrictions associées nous ont imposé de passer des entretiens en face à face aux enquêtes téléphoniques pour la collecte des données finales. Les enquêtes menées auprès des filles âgées de 12 à 19 ans ont dû être écourtées et réparties sur deux appels téléphoniques distincts. Les enquêtes téléphoniques ont probablement introduit un biais en faveur des filles plus privilégiées et des familles ayant un meilleur accès au téléphone. Le rapport d'évaluation à mi-parcours (Melnikas et al., 2019) évoque des difficultés au niveau méthodologique liées à la randomisation et à la mise en œuvre du programme. Par conséquent, nous présentons des résultats ajustés pour le Malawi, le Mali et le Niger. En Inde, l'échantillon était équilibré pour l'étude de base. Par conséquent nous présentons des résultats non ajustés.

La mise en œuvre de programmes multisectoriels dans des contextes très variés où le mariage des enfants est très répandu ou représente un lourd fardeau pour les filles et leurs familles, permet au programme MNCP d'apporter une contribution importante en utilisant les résultats d'un modèle de programme similaire mis en œuvre dans divers contextes. Les quatre pays inclus dans l'évaluation du programme MNCP variaient considérablement en termes d'efforts dans la prévention du mariage des enfants et de réalisations précédentes. L'évaluation porte sur l'Inde, où la prévalence du mariage des enfants a diminué au cours de la dernière décennie, et sur le Niger, où elle reste obstinément élevée. Ces contextes diffèrent également par des facteurs spécifiques du mariage des enfants qui peuvent ne pas être des facteurs dans d'autres contextes, les rapports sexuels, par exemple, et les grossesses avant le mariage au Malawi et au Mali. Les résultats de cette évaluation remettent également en question l'hypothèse selon laquelle il est plus facile pour les programmes de démontrer l'efficacité de la réduction du mariage des enfants dans les zones où le mariage des enfants est le plus élevé. Même dans les zones à faible 
prévalence en Inde, le programme a montré son impact. Globalement, nos données suggèrent que des programmes tels que le programme MNCP peuvent contribuer de manière significative à mettre fin à la pratique du mariage des enfants, même dans les régions où elle est en déclin. Ces résultats sont prometteurs et devraient encourager la poursuite des efforts afin d'apporter des changements positifs dans la vie des adolescentes vivant dans ces contextes difficiles. 


\section{Liste des abréviations}

EDD Écarts dans les différences

EDS Enquête démographique et de santé

FNUAP Fonds des Nations Unies pour la population

GHV Group Head Village (unité administrative au Malawi)

GNB Girls Not Brides (Filles pas épouses)

GP Gram Panchayats

MNCP Marriage: No Child's Play (Le mariage n'est pas un jeu d'enfant)

MTBA Alliance « More than Brides »

NFHS National Family Health Survey (Enquête nationale sur la santé familiale)

PSU «Primary sampling unit » (Unité d'échantillonnage primaire)

SDSR Santé et droits sexuels et reproductifs

UNICEF Fonds des Nations Unies pour l'enfance

ZD Zone de dénombrement 


\section{CHAPITRE 1 \\ Introduction}

\section{Contexte}

Au niveau mondial, le mariage des enfants reste un problème important pour la santé et les droits de l'homme. Malgré la récente baisse du nombre de mariages d'enfants dans le monde - de $25 \%$ de filles mariées avant l'âge de 18 ans à $21 \%$ entre 2008 et 2018 (UNICEF 2018) - on estime que 12 millions de filles sont encore mariées chaque année (GNB 2021). Le mariage des enfants a pour les filles des conséquences telles qu'une éducation écourtée, des risques sanitaires liés à une naissance précoce, une incidence plus élevée de la violence entre les partenaires et des problèmes de santé mentale (Hindin et al., 2008; Mensch et al., 1998; Gage, 2013). Au niveau sociétal, la lutte contre le mariage des enfants est à la fois un bienfait social - le mariage des enfants est une question qui relève des droits de l'homme (Nour 2009) - et un impératif économique : selon les estimations, rien qu'au Niger, l'éradication du mariage des enfants permettrait de réaliser des gains de bien-être annuels de l'ordre de 1,7 milliard de dollars d'ici 2030 (Wodon et al., 2017).

\section{La pauvreté et l'incertitude économique} sont fréquemment citées comme des

raisons du mariage précoce par des

voies impliquant l'insécurité

alimentaire, les coûts de l'éducation, ou

des coûts dot plus élevés associés aux

mariages de filles plus âgées.
Si les causes du mariage des enfants sont propres à chaque contexte, nous savons que la paurreté et l'inégalité entre les sexes sont des facteurs déterminants dans tous les contextes (GNB 2021). La pauvreté et l'incertitude économique sont fréquemment citées comme des raisons du mariage précoce par des voies impliquant l'insécurité alimentaire, les coûts de l'éducation ou les coûts de la dot associés à un âge plus avancé du mariage (Amin et al. 2018, Alston et al., 2014, Hoogeveen, et al., 2011). Les données suggèrent également que les grossesses avant le mariage et les préoccupations relatives à la sécurité sexuelle (Greene et al. 2018), les chocs économiques et sociaux tels que le changement climatique et les déplacements (Alston et al 2014 ; Hoogeveen et al 2011 ; Andriano \& Behrman 2020), les catastrophes naturelles (Felton-Biermann 2006) et les pandémies (Bandiera et al, 2018), y compris la pandémie de Covid-19 (UNFPA 2020), menacent également d'exacerber le mariage des enfants et de bloquer les progrès réalisés ces dernières années.

Au cours des vingt dernières années, les interventions visant à retarder le mariage des filles ont suscité une attention accrue. Une série d'examens systématiques (Lee-Rife et al., 2012 ; Kalamar et al., 2016 ; Chae \& Ngo, 2017 ; Malhotra \& Elnakib, 2021) ont déterminé les approches les plus efficaces pour réduire le mariage des enfants. Ces examens ont démontré que peu de programmes ont été évalués de manière rigoureuse - souvent moins de 20. Parmi les programmes qui répondent aux critères d'inclusion dans ces études, les programmes 
d'autonomisation et les approches axées sur les moyens de subsistance semblent être les plus prometteurs.

L'examen systématique le plus récent sur le mariage des enfants (Malhotra \& Elnakib, 2021) a intégré 13 études supplémentaires publiées depuis les examens précédents décrits ci-dessus et a examiné la durabilité en plus de l'impact du programme sur le mariage des enfants. Les auteurs en ont conclu que les transferts d'argent pour soutenir l'éducation des filles sont l'intervention la plus efficace pour réduire le mariage des enfants à ce jour en raison de « l'amélioration du capital humain propre aux filles ». Les programmes augmentant les opportunités économiques visibles pour les filles et les femmes enregistrent également un certain succès.

Les preuves disponibles présentent certaines limites qui laissent encore des questions importantes pour ceux qui conçoivent des programmes visant à réduire le mariage des enfants. Bon nombre des interventions réussies à ce jour ont testé des interventions spécifiques dans un pays et parfois dans un secteur (par exemple, l'éducation, la santé, les moyens de subsistance) pour comprendre l'effet de cette composante. Les programmes à composantes multiples n'ont généralement pas été aussi efficaces que les programmes à composante unique, comme le démontre l'ensemble des données sur les programmes relatifs au mariage des enfants (Malhotra \& Elnakib, 2021), alors que les facteurs du mariage des enfants sont multisectoriels. De plus, certains pays (par exemple, l'Inde, le Bangladesh) sont fortement représentés dans les revues systématiques alors que d'autres contextes (par exemple, l'Afrique de l'Ouest) ont peu d'évaluations rigoureuses répondant aux critères d'inclusion. La mesure dans laquelle les résultats peuvent être transposés d'un contexte à l'autre reste incertaine.

\section{Partenaires du programme MNCP et contextes d'intervention}

De 2016 à 2020, l'Alliance More Than Brides (MTBA) - un consortium composé de Save the Children Netherlands, Simavi et Oxfam Novib en tant que partenaires internationaux de mise en œuvre, de 25 partenaires locaux de mise en œuvre et de Population Council en tant que partenaire de recherche - a mis en œuvre et évalué le programme MNCP (Marriage : No Child's Play) dans certaines régions de l'Inde, du Malawi, du Mali, du Niger et du Pakistan. Population Council a dirigé l'évaluation de l'impact en Inde, au Malawi, au Mali et au Niger, tandis qu'Oxfam Novib a mené une évaluation distincte au Pakistan. D'un pays à l'autre, le programme MNCP varie considérablement en termes de nombre d'organisations partenaires locales de mise en œuvre (de 2 au Malawi à 12 en Inde) et de nombre de villages d'intervention (de 42 au Mali et au Niger à 609 en Inde). Le Tableau 1 fournit des détails sur les localités d'intervention dans chaque pays inclus dans l'évaluation d'impact. Consulter l'annexe 1 pour une liste complète des organisations partenaires de la MTBA impliquées dans la mise en œuvre du programme MNCP dans chaque localité.
Tableau 1. Pays, régions, et nombre de villages où le programme MNCP a été mis en œuvre

\begin{tabular}{|c|c|c|}
\hline Pays & Région & $\begin{array}{r}\text { Nombre de } \\
\text { villages }\end{array}$ \\
\hline \multirow{4}{*}{ Inde } & Jharkhand & 279 \\
\hline & Bihar & 75 \\
\hline & Odisha & 210 \\
\hline & Rajasthan & 45 \\
\hline \multirow{2}{*}{ Malawi $^{\star}$} & Mangochi & 108 \\
\hline & Nkhata Bay & 45 \\
\hline \multirow{2}{*}{ Mali } & Ségou & 30 \\
\hline & Sikasso & 12 \\
\hline \multirow{2}{*}{ Niger } & Maradi & 10 \\
\hline & Tillabéri & 32 \\
\hline \multicolumn{3}{|c|}{$\begin{array}{l}\text { * Le programme MNCP a également été mis en œuvre à Mchinji, } \\
\text { mais cette partie du programme a été exclue de la recherche en } \\
\text { raison de la présence d'une initiative de subsistance à grande échelle }\end{array}$} \\
\hline \multicolumn{3}{|c|}{$\begin{array}{l}\text { urnit des détails sur les localités d'intervention } \\
\text { ct. Consulter l'annexe } 1 \text { pour une liste complète } \\
\text { quées dans la mise en œuvre du programme }\end{array}$} \\
\hline
\end{tabular}


Le projet MNCP et son évaluation contribuent à la formation du corpus de documents sur les interventions efficaces permettant de retarder le mariage, et de mettre en œuvre et d'évaluer ces interventions dans divers contextes, du pays ayant la prévalence de mariage d'enfants la plus élevée au monde (Niger) au pays ayant le nombre absolu de mariages d'enfants le plus élevé (Inde), ainsi que dans des contextes où les relations sexuelles avant le mariage sont courantes et souvent un moteur du mariage des enfants (Malawi) et dans des contextes où l'initiation sexuelle a lieu presque exclusivement au sein du mariage (Inde et Niger). Les pays inclus dans cette évaluation ont des taux relatifs au mariage des enfants différents (Tableau 2) et dans certains cas, des moteurs différents. Bien qu'un examen approfondi de l'économie politique de chaque pays dépasse le cadre de ce projet, nous soulignons certains indicateurs clés susceptibles d'avoir des répercussions sur le mariage des enfants et les programmes visant à lutter contre cette pratique dans chaque contexte (Tableau 3).

Tableau 2. Indicateurs clés sur le mariage des enfants, pays du programme MNCP

\begin{tabular}{lrrrr}
\hline & $\begin{array}{r}\text { Proportion de femmes de } 20 \\
\text { à } 24 \text { ans mariées à l'âge de } 15 \\
\text { ans }\end{array}$ & $\begin{array}{r}\text { Proportion de femmes de } \\
20 \text { à } 24 \text { ans mariées à l'âge } \\
\text { de } 18 \text { ans }\end{array}$ & $\begin{array}{r}\text { Classement : Prévalence } \\
\text { du mariage des enfants }\end{array}$ & $\begin{array}{r}\text { Âge minimum légal du } \\
\text { mariage pour les filles }\end{array}$ \\
\hline Inde & $7 \%$ & $27 \%$ & 12 & 18 \\
\hline Malawi & $9 \%$ & $42 \%$ & 5 & 18 \\
\hline Mali & $16 \%$ & $54 \%$ & 1 & 16 \\
\hline Niget in top 20] & $28 \%$ & $76 \%$ & 15 \\
\hline
\end{tabular}

Source : Statistiques sur les mariages provenant des enquêtes EDS ; informations sur le classement, les taux et la législation provenant de Girls Not Brides.

Tableau 3. Autres indicateurs contextuels clés, pays du programme MNCP

\begin{tabular}{|c|c|c|c|c|c|c|}
\hline Pays & $\begin{array}{r}\% \text { de femmes de } 20 \text { à } \\
24 \text { ans ayant eu leurs } \\
\text { premiers rapports } \\
\text { sexuels avant l'âge de } \\
18 \text { ans }\end{array}$ & $\begin{array}{r}\% \text { de ménages } \\
\text { disposant d'un } \\
\text { téléphone } \\
\text { portable }\end{array}$ & $\begin{array}{r}\text { \% de femmes de } 15 \text { à } 49 \\
\text { ans n'ayant pas travaillé } \\
\text { pour gagner leur vie au } \\
\text { cours des } 12 \text { derniers } \\
\text { mois }\end{array}$ & $\begin{array}{r}\% \text { de femmes } \\
\text { de } 15 \text { à } 49 \text { ans } \\
\text { sans éducation }\end{array}$ & $\begin{array}{l}\text { PIB } 2019 \text { par } \\
\text { habitant, USD }\end{array}$ & $\begin{array}{r}\text { Rang IDH } \\
2020 \\
\text { (de 189) }\end{array}$ \\
\hline Inde & $22 \%$ & $90 \%$ & $70 \%$ & $27 \%$ & 2120 & 131 \\
\hline Malawi & $57 \%$ & $50 \%$ & $33 \%$ & $12 \%$ & 380 & 174 \\
\hline Mali & $67 \%$ & $89 \%$ & $42 \%$ & $65 \%$ & 870 & 184 \\
\hline Niger & $73 \%$ & $50 \% *$ & $71 \%$ & $80 \%$ & 600 & 189 \\
\hline
\end{tabular}

\section{Interventions dans le cadre du programme MNCP}

\section{Composantes du programme MNCP}

L'approche du programme MNCP comprend un ensemble d'interventions communautaires mises en œuvre à plusieurs niveaux et dans plusieurs domaines. Dans tous les contextes, le programme 
a pour but d'autonomiser les filles, de les sensibiliser aux risques du mariage précoce, d'améliorer l'accès des filles aux services de santé sexuelle et reproductive et de soutenir les normes sociales favorables à l'éducation des filles, à leur engagement économique et à leur participation aux décisions conjugales. Le programme MNCP est axé sur la

Le programme MNCP vise à être holistique et à cibler les voies menant au mariage des enfants à plusieurs niveaux. communauté et comprend des activités liées aux sept domaines de résultats clés suivants :

- Renforcer l'autonomie des adolescentes à risque et déjà mariées grâce à une éducation aux compétences de la vie courante, aux informations sur la santé et les droits sexuels et reproductifs et aux groupes de soutien par les pairs ;

- Renforcer l'accès des filles à l'éducation et améliorer leur maintien à l'école ;

- Améliorer l'accès des filles et de leurs familles aux opportunités économiques et génératrices de revenus ;

- Renforcer l'accès aux systèmes améliorés de protection de l'enfance ;

- Accroître l'accès à des services de SDSR de qualité et adaptés aux jeunes ;

- Contribuer à l'évolution des normes sociales qui perpétuent la pratique du mariage des enfants ; et

- Influencer les cadres juridiques et politiques.

Le programme MNCP vise à être holistique et à cibler les voies menant au mariage des enfants à plusieurs niveaux simultanément. Il a également établi un classement entre les communautés ayant bénéficié du programme MNCP complet et les communautés n'ayant fait l'objet d'aucune intervention. L'intervention a été adaptée aux défis et aux moteurs spécifiques du mariage des enfants dans chaque contexte. Ces adaptations ont nécessité une modification significative du programme selon les pays et les états/régions. La Figure 1 ci-dessous illustre les éléments clés de l'intervention du MNCP dans les différents contextes.

Figure 1. Composantes du programme à différents niveaux

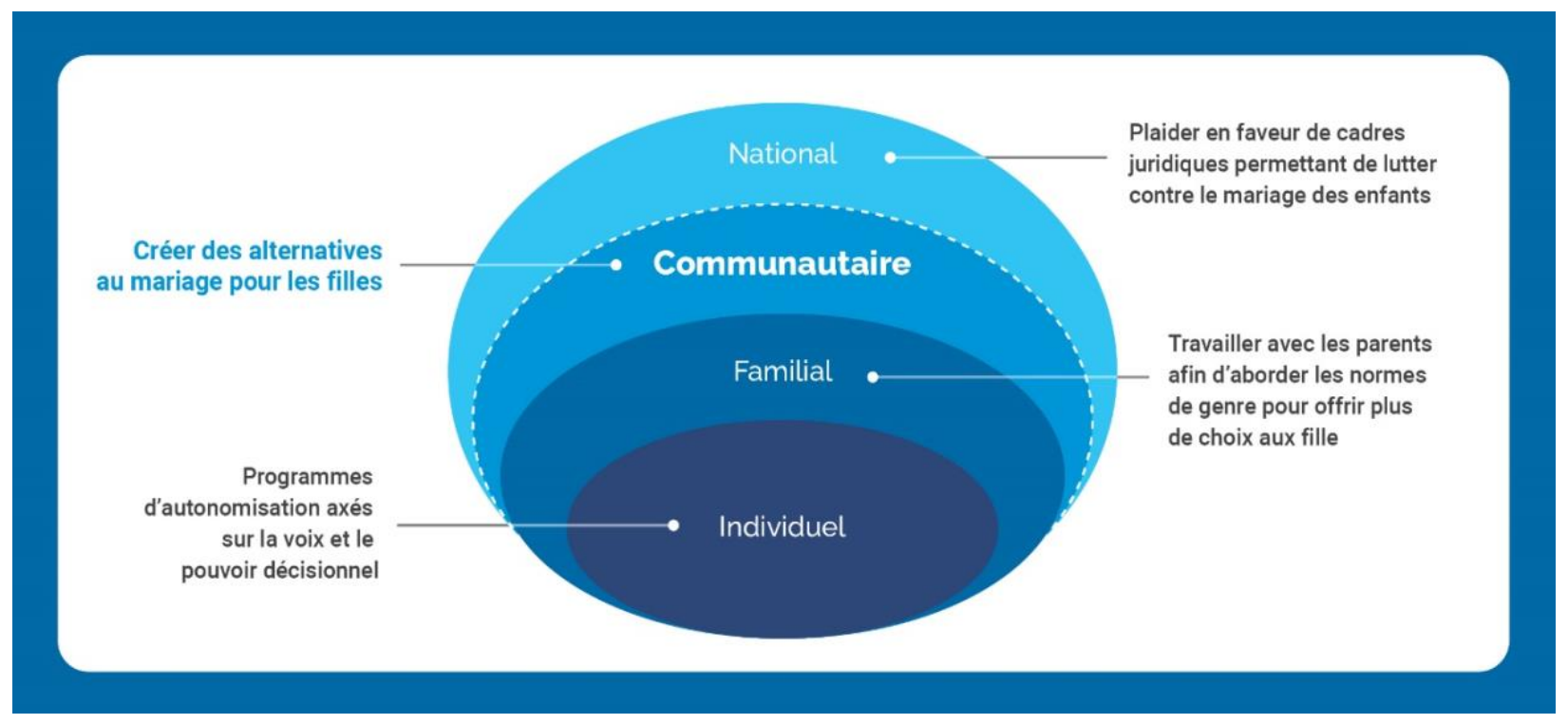


Adaptations de la programmation à la pandémie Covid-19

Au cours de la dernière année de programmation, les stratégies du programme MNCP ont dû être adaptées en raison de la pandémie de Covid-19 et des restrictions connexes en matière de déplacements et de rassemblements. Au cours de cette période, certaines activités prévues notamment celles impliquant des groupes importants - ont été suspendues ou modifiées de manière significative afin de limiter le nombre de participants. Dans certains pays, les activités prévues avec ou pour les représentants du gouvernement ont été suspendues ou abrégées, en raison des priorités concurrentes du personnel gouvernemental pendant la crise en cours. D'autres activités du programme MNCP ont cependant pu être adaptées. À titre d'exemple, les partenaires de mise en œuvre ont maintenu la communication avec les mentors, les groupes de jeunes, les comités communautaires de protection de l'enfance et d'autres parties prenantes du programme par le biais d'appels téléphoniques, de SMS, de messages WhatsApp, de Facebook et/ou d'émissions de radio afin de garder les réseaux actifs, de partager des informations sur les thèmes du programme ainsi que sur la Covid-19, et de coordonner les efforts de soutien matériel direct dans certains contextes, notamment la fourniture de serviettes hygiéniques, de désinfectant pour les mains et de masques. Bien que la plupart de ces méthodes de communication à distance aient déjà été utilisées dans le cadre du programme MNCP, l'utilisation et la dépendance à l'égard de la technologie ont considérablement augmenté pendant la pandémie.

\section{Conception et objectifs de la recherche du programme MNCP}

\section{Composantes de la recherche du programme MNCP}

La recherche menée dans le cadre du programme MNCP comprend la collecte de données quantitatives et qualitatives afin d'informer sur la mise en œuvre du programme et d'évaluer son impact. Comme le montre la Figure 2 ci-dessous, une étude de base a été menée dans chaque contexte avant la mise en œuvre des programmes, une étude à mi-parcours a été menée en 2018, deux ans après la mise en œuvre du programme, et une étude finale a été menée à la fin de la période de mise en œuvre du programme. Lors de chaque période, les données ont été collectées par le biais d'enquêtes transversales menées auprès des adolescentes de 12 à 19 ans dans les zones d'intervention du programme et dans les zones de comparaison où il n'y a pas eu d'engagement direct du programme MNCP. Ce rapport présente les principaux résultats de ces enquêtes. Des informations plus détaillées sur la conception de l'évaluation sont disponibles dans le rapport d'évaluation à mi-parcours de la MTBA (Melnikas et al., 2019). Les recherches qualitatives sont disponibles sur les sites Internet du Population Council et du projet More than Brides (Population Council, 2016; More than Brides, 2020). 
Figure 2 : Calendrier de recherche et d'évaluation du programme MNCP

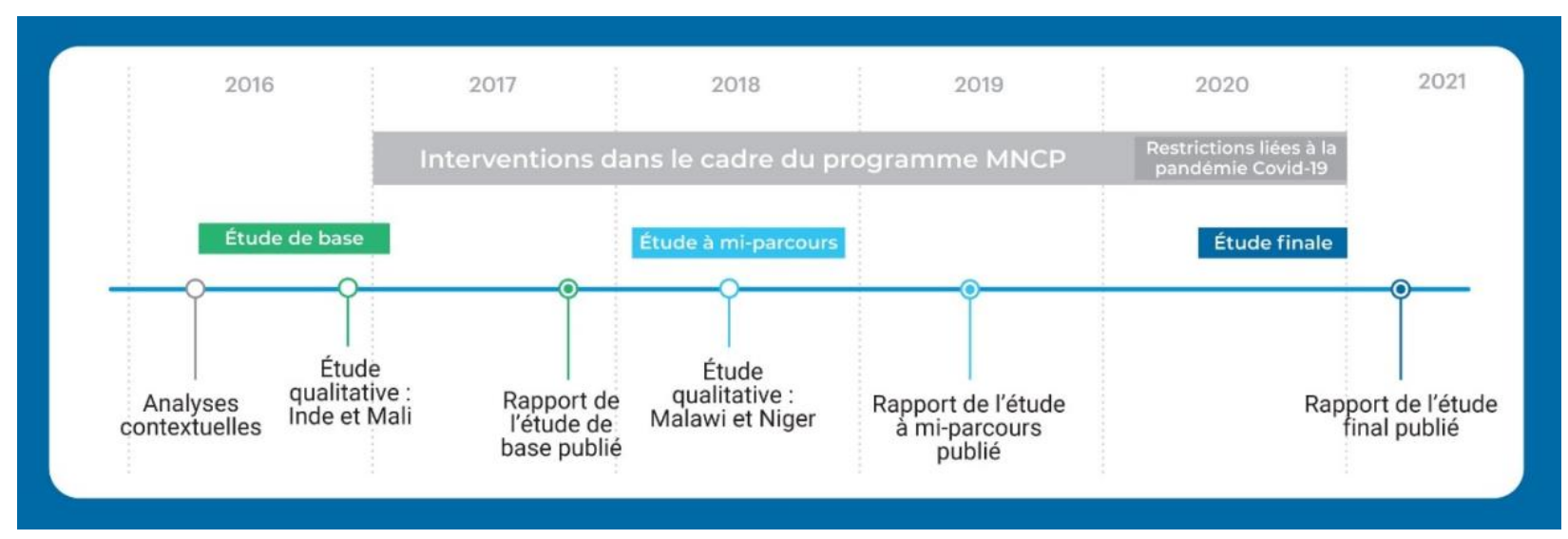

Résumé des résultats de l'étude à mi-parcours

L'évaluation à mi-parcours du programme MNCP, réalisée en 2018, a révélé que le mariage des enfants avait diminué sur la totalité des échantillons depuis le début de l'étude. Nous avons observé des baisses allant de $29 \%$ à $47 \%$ dans tous les pays (soit des baisses de 3 à 11 points de pourcentage dans l'ensemble); cependant, nous n'avons pas été en mesure de conclure que la baisse du nombre de mariages d'enfants était directement liée au programme. Nous avons plutôt observé ces tendances dans les communautés d'intervention et de comparaison, reflétant des tendances plus larges au niveau mondial (UNICEF, 2018). Les résultats à mi-parcours indiquent effectivement certaines améliorations attribuables au programme en matière de connaissances concernant le mariage des enfants et la SDSR dans les zones d'intervention entre le début de l'étude (2016) et l'étude à mi-parcours (2018) (Melnikas et al., 2019). Au Niger et au Malawi, les connaissances en matière de contraception moderne ont fortement augmenté dans les zones d'intervention (de $33 \%$ au Niger et de $29 \%$ au Malawi), tandis que dans les zones de comparaison, les connaissances en matière de contraception n'ont que légèrement augmenté (de $3 \%$ au Malawi), voire même diminué (de $3 \%$ au Niger). Les résultats à mi-parcours ont également montré que la connaissance de l'âge légal du mariage s'était améliorée dans les zones d'intervention et de comparaison au Malawi et dans trois états indiens (Bihar, Jharkhand et Rajasthan), mais qu'elle n'était attribuable au programme que dans un seul état de l'Inde (Odisha). Nous n'avons pas constaté une amélioration des indicateurs d'éducation ou des moyens de subsistance directement liée à l'intervention MNCP, mais ces indicateurs avaient une tendance positive à mi-parcours.

\section{Objectifs de l'évaluation finale et adaptations liées à la Covid-19}

Dans ce rapport final, nous examinons les résultats du programme MNCP après plus de quatre années de mise en œuvre, y compris au cours de la dernière année, caractérisée par d'importantes adaptations dues à la pandémie de Covid-19 tant dans la programmation que dans la recherche. En mars 2020, nous avons interrompu la collecte des données pour l'étude finale car la pandémie s'est répandue au niveau mondial. Afin de minimiser les déplacements et les contacts en face à face, les données ont été collectées par téléphone (avec des instruments nettement plus courts). Sachant que les restrictions ont probablement perturbé les tendances positives sur les indicateurs de SDSR, de l'éducation et du mariage, observées lors de l'évaluation à mi-parcours en 2018, nous avons ajouté un module supplémentaire afin de comprendre les répercussions de 
la pandémie et de la fermeture des établissements scolaires sur les finances des ménages, l'éducation des filles, les projets de mariage et la santé mentale et physique.

Les études finales ont été conçues pour produire des données comparables dans tous les contextes. L'impact du programme a été évalué par rapport aux groupes de comparaison. Les comparaisons entre les études de base, à mi-parcours et finales servent à décrire les tendances dans les zones d'intervention et de comparaison. L'évaluation est donc en mesure de fournir des estimations de l'impact et des tendances du programme de manière comparable dans des contextes qui diffèrent considérablement en termes de taux et de prévalence du mariage des enfants, ainsi que de facteurs du mariage des enfants tels que les relations sexuelles avant le mariage, les modalités du mariage et la pauvreté. Le rapport explore les résultats comportementaux tels que le mariage des enfants, la scolarité, le travail et la grossesse, ainsi que les indicateurs de connaissances et d'attitudes. 


\section{Structure du rapport final}

Le chapitre 1 offre une vue d'ensemble du problème du mariage des enfants et une description des objectifs du programme. Le chapitre 2 donne un aperçu des méthodes, il explique notamment la façon dont la recherche s'est adaptée après l'étude à mi-parcours en raison des limites de la collecte de données en personne. Dans le chapitre 3 , nous examinons les résultats de l'étude finale sur l'impact de la pandémie de Covid-19 et des restrictions associées sur le bien-être des adolescentes et de leurs familles et proposons des voies par lesquelles la pandémie de Covid-19 et les restrictions associées peuvent influencer le mariage des enfants. Dans les chapitres 4 à 7 , nous examinons les résultats par pays en nous concentrant sur l'impact du programme sur les indicateurs clés, notamment le mariage des enfants, l'éducation, les connaissances et l'accès aux services SDSR, les activités de subsistance et les attitudes équitables entre les sexes. Enfin, au chapitre 8 , nous examinons les résultats de l'étude finale dans les quatre contextes, nous reconnaissons les limites et les difficultés de l'étude à démontrer l'impact, et nous considérons les implications pour les futurs programmes et recherches sur le mariage des enfants. 


\section{CHAPITRE 2 \\ Méthodes}

\section{Conception de l'évaluation}

La conception MTBA originale visait à mesurer le changement au niveau de la communauté à trois moments (au début de l'étude en 2017, à mi-parcours en 2018 et à la fin de l'étude en 2020) en comparant les niveaux globaux de mariage, d'éducation, d'activités de subsistance, de connaissances et de comportements liés à la santé et aux droits sexuels et reproductifs dans les zones d'intervention et de comparaison. Des informations spécifiques sur la méthodologie de base, y compris la sélection des sites et la randomisation par grappes, sont disponibles dans une série de rapports de base (Population Council, 2016). Après la collecte des données de miparcours en 2018, nous avons mesuré l'impact du programme avec la méthode de l'écart dans les différences (EDD), en comparant les changements dans les zones d'intervention entre le début de l'étude et l'étude à mi-parcours par rapport aux changements dans les zones de comparaison au cours de la même période. À la fin de la période, nous utilisons également l'EDD, mais nous comparons les changements survenus du début à la fin de l'étude dans les zones d'intervention par rapport aux changements survenus dans les zones de comparaison.

\section{Mode de collecte des données}

En raison de la pandémie de Covid-19 et de la suspension des activités de collecte de données en personne, les entretiens finaux en face à face prévus avec les adolescentes n'ont pu être réalisés en 2020. Nous avons dû chercher une alternative à cette approche qui permettrait tout de même de comprendre l'impact du programme et d'évaluer les répercussions de la Covid-19 sur les indicateurs de résultats clés. Après avoir commencé la collecte de données finale en Inde et au Mali en février 2020, nous avons rappelé les équipes de terrain début mars en raison de risques sanitaires potentiels liés à la Covid-19. Comme la pandémie a continué à se répandre au niveau mondial, nous avons décidé, à la mi-2020, d'effectuer des collectes de données à distance afin de continuer à mener des enquêtes auprès des adolescentes des communautés échantillonnées et de comprendre les résultats clés du programme. Nous avons ajouté des entretiens avec des parents d'adolescentes ou d'autres membres adultes de la famille des adolescentes afin de mesurer l'impact de la pandémie de Covid-19 au niveau du ménage.

\section{Instruments}

Le changement méthodologique de la collecte des données a nécessité le passage d'un instrument d'enquête plus long, qui recueillait des indicateurs socio-économiques au niveau du ménage ainsi que des indicateurs dans plusieurs domaines, à une série d'instruments plus courts, en partie à cause de la difficulté d'effectuer de longues enquêtes téléphoniques auprès des adolescentes dans des contextes à faibles ressources. Nous avons divisé l'enquête auprès des adolescentes en deux instruments et ajouté une enquête auprès des adultes pour recueillir des informations sur le ménage. En outre, les deux enquêtes comprenaient des informations 
spécifiques à la Covid-19 afin de comprendre les répercussions de la pandémie et de la fermeture des établissements scolaires sur les adolescentes et leurs familles.

\section{Échantillonage}

Parce qu'au départ et à mi-parcours, nous avons dressé une liste de ménages et ré-échantillonné les filles de 12 à 19 ans dans des communautés sélectionnées afin d'évaluer l'impact de l'intervention au niveau de la communauté (plutôt que de suivre les filles de manière prospective), nous avons dû changer notre méthodologie d'échantillonnage en fin d'étude dans la mesure où l'enquête en face à face auprès de milliers de ménages a été jugée dangereuse. Nous avons d'abord essayé d'échantillonner les filles en sélectionnant un point de contact central dans la communauté (dans ce cas, un agent de santé) susceptible de nous aider à localiser les filles éligibles. Bien que cette approche ait fonctionné dans certaines communautés indiennes, nous n'avons pas été en mesure de répertorier un nombre suffisant de filles dans chaque région et avons donc procédé à un échantillonnage à partir du recensement final de février 2020 (Inde) et des recensements à mi-parcours de 2018 (Malawi, Mali et Niger), en sélectionnant les filles éligibles qui auraient 12-19 ans à la fin de l'enquête. Les filles qui devraient avoir entre 12 et 19 ans à la fin de l'enquête ont été sélectionnées de manière aléatoire à partir des données du recensement à mi-parcours afin de créer des échantillons originaux et de remplacement pour chaque région. Nous avons obtenu les coordonnées des parents ou du tuteur de chaque fille sélectionnée afin de les inviter à participer à l'enquête auprès des adultes. Notre objectif était d'interroger les parents ou d'autres membres adultes de la famille d'au moins la moitié des filles de l'échantillon.

Remplacement: Parce que nous avons échantillonné des filles pour leur participation à l'étude finale à partir d'une liste de ménages dressée à mi-parcours en 2018, nous avons également échantillonné des remplaçantes afin que les recenseurs puissent trouver un nombre adéquat de filles pour l'enquête. Dans la plupart des contextes, nous avons échantillonné une liste aléatoire de remplaçantes à utiliser en cas de besoin, en commençant par le haut de la liste et en allant vers le bas. Nous avons prévu qu'au cours des deux années écoulées depuis la collecte des données à mi-parcours, les filles pouvaient avoir quitté le village. Au Mali, en raison du calendrier de l'enquête et des déplacements importants d'adolescentes depuis l'enquête de mi-parcours, nous avons sur-échantillonné des remplaçantes pour chaque village et, dans certains cas, nous avons dû parcourir entièrement la liste échantillonnée afin de recueillir suffisamment de réponses. Dans les villages où l'équipe n'a pas été en mesure de répertorier plus de 20 filles (8 villages à Ségou, Mali), les filles ont été sur-échantillonnées dans les villages appariés par statut d'intervention/comparaison, à proximité de la grande ville la plus proche.

Questions d'échantillonnage : Au Niger, en raison de problèmes de sécurité, 8 villages ont été exclus de la collecte de données. Pour compenser, les zones jugées les plus similaires en termes d'affectation et de composition ethnique ont été sur-échantillonnées. Au Malawi, 3 zones de dénombrement (ZD) ont été exclues en raison du nombre insuffisant de filles éligibles de 12 à 19 ans d'après les données de mi-parcours. Comme au Niger, nous avons sur-échantillonné les filles dans des régions similaires en termes d'affectation et de composition ethnique dans d'autres ZD afin de maintenir l'équilibre entre les zones d'intervention et de comparaison. 


\section{Collecte des Données}

Les données ont été recueillies par des équipes de recherche expérimentées (CERIPS au Mali, LASDEL au Niger, IKI au Malawi et Population Council India en Inde) qui avaient également recueilli des données pour les études de base (2016/7) et à mi-parcours (2018). Les répondants éligibles ont été échantillonnés à partir des données figurant sur la liste et ont reçu la visite des enquêteurs en personne qui ont demandé le numéro de téléphone de l'adolescent répondant et celui d'un adulte proche (parent, tuteur ou autre membre adulte du ménage). Le consentement initial et la permission ont également été obtenus à ce moment-là. Dans certains cas, et selon les directives du comité d'éthique local, des incitations telles que des masques réutilisables ont été fournies au moment de l'inscription sur la liste. Une fois l'exercice de listage téléphonique terminé, les enquêteurs ont mené des enquêtes auprès d'adolescentes (chapitres 1 et 2) et d'adultes à distance depuis des lieux privés. Des incitations telles que des temps d'antenne ont été fournies aux participants ou aux ménages (selon les directives du comité d'éthique local) après la réalisation des enquêtes. Les données ont été collectées à l'aide de tablettes, de téléphones et de l'outil mobile de collecte de données sur une plateforme en nuage assistée par SurveyCTO. Des identifiants uniques ont été attribués aux ménages et chaque enquête a été connectée par cet identifiant. Le Tableau 4 ci-dessous indique la taille des échantillons par enquête.

Tableau 4. Échantillons de référence, de mi-parcours, finaux, pays MNCP

\begin{tabular}{|c|c|c|c|c|c|c|}
\hline Pays & État/Région & $\begin{array}{r}\text { Étude de base } \\
\text { auprès des } \\
\text { adolescentes }\end{array}$ & $\begin{array}{r}\text { Étude à mi-parcours } \\
\text { auprès des } \\
\text { adolescentes }\end{array}$ & $\begin{array}{r}\text { Étude finale } 1 \\
\text { auprès des } \\
\text { adolescentes }\end{array}$ & $\begin{array}{r}\text { Étude finale } 2 \\
\text { auprès des } \\
\text { adolescentes }\end{array}$ & $\begin{array}{r}\text { Étude finale } \\
\text { auprès des } \\
\text { adultes }\end{array}$ \\
\hline & & 2016-17 & 2018 & & 2020 & \\
\hline Inde & $\begin{array}{l}\text { Bihar, Jharkhand, } \\
\text { Rajasthan, Odisha }\end{array}$ & 2982 & 2801 & 1,479 & 1479 & 1,479 \\
\hline Malawi & $\begin{array}{l}\text { Mangochi, } \\
\text { Nkhata Bay }\end{array}$ & 1020 & 1029 & 764 & 764 & 786 \\
\hline Mali & Sikasso, Ségou & 855 & 829 & 819 & 816 & 479 \\
\hline Niger & Maradi, Tillabéri & 600 & 599 & 620 & 620 & 329 \\
\hline
\end{tabular}

\section{Revue éthique}

Les autorisations d'éthique et de recherche pour la présente étude ont été délivrées par le Conseil d'examen institutionnel du Population Council et par l'Institut national de recherche en sciences humaines et sociales à Lilongwe (Malawi), l'Institut national de recherche en santé publique (INRSP) (Mali), et le Comité d'éthique pour la recherche en santé (Niger).

\section{Analyse des données}

Nous avons effectué l'analyse EDD à l'aide de Stata SE 14.2, ajustée pour la conception par grappes. En Inde et au Malawi, où les sites d'intervention étaient randomisés et équilibrés au départ, nous n'avons pas ajusté les covariables. Pour l'Inde, nous présentons donc les résultats non ajustés de l'EDD. Au Malawi, nous ne procédons à aucun ajustement pour les covariables, mais nous reconnaissons que la mise en œuvre du programme a légèrement dévié de la randomisation, certaines zones n'ayant pas bénéficié de l'intervention et certaines zones de comparaison ayant été traitées. Nous présentons donc les résultats « tels que mis en œuvre » au 
Malawi, qui reflètent plus fidèlement l'impact du programme. Au Mali et au Niger, l'analyse EDD a inclus des covariables car la conception de la recherche était quasi-expérimentale (zones de comparaison appariées) et les zones d'intervention et de comparaison différaient au départ. L'analyse EDD pour le Mali et le Niger est ajustée en fonction de l'âge, du niveau d'éducation, de la richesse et de l'ethnicité. L'âge et le niveau d'éducation ont été inclus au niveau individuel sur la base des réponses à l'enquête menée auprès des adolescentes. Les données sur la richesse des ménages et l'appartenance ethnique des filles n'ont pas été collectées dans le cadre des études finales. Pour cette raison, la richesse et l'ethnicité ont été mesurées au niveau de la communauté sur la base des réponses des adultes et des enquêtes précédentes. L'ethnicité a été mesurée en tant que proportion de la communauté appartenant au groupe ethnique dominant. La richesse a été mesurée en tant que niveau moyen de richesse au sein de la communauté. Pour les études de base et à mi-parcours, la richesse a été mesurée à partir des années d'étude respectives. Quant à l'étude finale, la richesse a été mesurée en tant que moyenne des réponses de référence et à mi-parcours au sein de la communauté. Des résultats détaillés supplémentaires des analyses EDD et des calculs de puissance sont disponibles dans les annexes 4 et 5 de la version anglaise de ce rapport, ce qui est disponible sur les sites Internet du Population Council et de l'alliance More Than Brides. 


\section{CHAPITRE 3 \\ Impact de la pandémie de Covid-19 sur les adolescentes}

\section{Perturbations liées à la pandémie de Covid-19}

Au-delà de ses coûts directs en termes de vies humaines, de santé et de la charge excessive imposée aux systèmes de santé, la pandémie de Covid-19 a provoqué des perturbations de grande ampleur. La Figure 3 ci-dessous indique les conséquences de la pandémie sur la vie des adolescentes.

Figure 3. Conséquences multidimensionnelles de la Covid-19

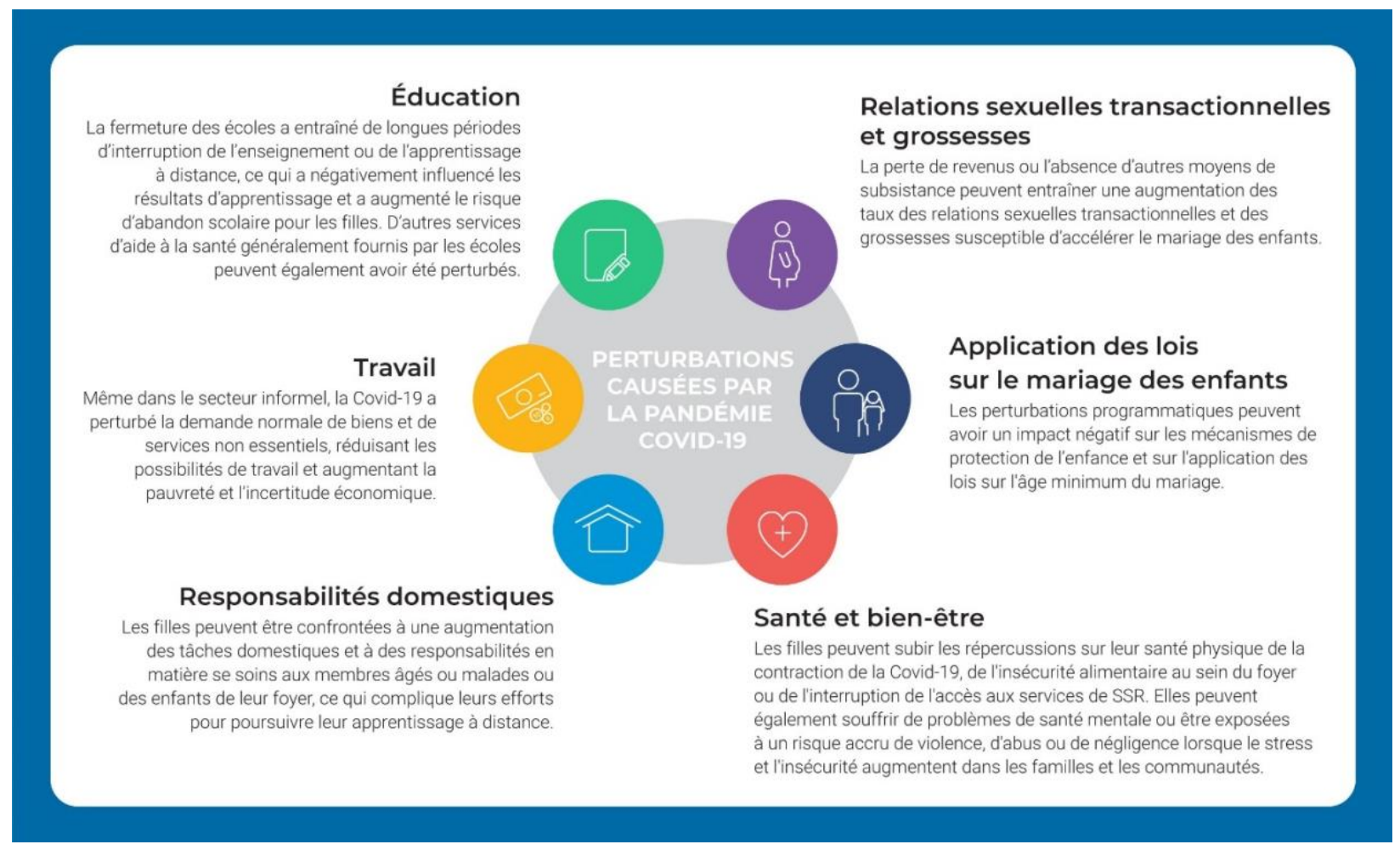

Dans l'évaluation finale, nous examinons l'influence de la Covid-19 sur la vie des adolescentes, notamment sur les finances du ménage et l'insécurité financière, l'interruption de l'éducation, les résultats en matière de santé physique et mentale, et les inégalités entre les sexes, notamment la violence sexiste.

\section{Impact de la Covid-19 sur le mariage des enfants}

Nous savons, grâce à des recherches antérieures sur les déplacements (Mourtada et al., 2014 ; Melnikas et al., 2020), le changement climatique (Alston et al., 2014) et les maladies infectieuses comme Ebola (Bandiera et al., 2018), que les crises politiques, environnementales et sanitaires exacerbent les facteurs connus du mariage des enfants, tels que la pauvreté et l'inégalité entre 
les sexes, et peuvent entraîner une augmentation des grossesses précoces et des mariages d'enfants. La pandémie de Covid-19 devrait freiner les progrès réalisés au niveau mondial en matière de réduction des mariages d'enfants et d'amélioration de l'équité entre les hommes et les femmes. Selon les estimations de I'UNFPA, 13 millions de mariages d'enfants supplémentaires résulteront des perturbations causées par la Covid-19 (UNFPA, 2020).

Étant donné que l'impact réel de la pandémie de Covid-19 sur le mariage des enfants ne sera en général pas détectable dans les données nationales à court terme, il est important de surveiller les facteurs à mi-parcours du mariage des enfants afin de mieux comprendre comment cette crise complexe aggrave les risques encourus par les filles. La Figure 4 ci-dessous indique les voies par lesquelles la pandémie de Covid-19 peut aggraver le mariage des enfants. Alors que la pauvreté et l'insécurité augmentent au niveau des ménages et des communautés en raison de maladies ou de décès au sein des familles, de la perte de revenus et de la volatilité des marchés économiques, les parents peuvent se sentir poussés à marier leurs filles plus tôt afin d'alléger la pression financière qui pèse sur eux ou de garantir l'avenir financier de leurs filles. Les perturbations des programmes et des services qui aident à soutenir les familles sur le plan économique et permettent aux adolescentes de rester à l'école peuvent aggraver la pression financière sur les familles et accroître la vulnérabilité des filles au mariage des enfants. La demande accrue de travail domestique et de soins au sein des ménages peut contribuer à maintenir les filles hors de l'école au-delà de la période de fermeture des établissements ou inciter les ménages à rechercher des jeunes mariées pour remplir ces rôles domestiques. La fermeture des établissements scolaires et les difficultés à poursuivre l'éducation à distance peuvent avoir un impact négatif sur les résultats d'apprentissage, augmenter l'incidence de l'abandon scolaire, réduire le niveau d'éducation des filles, et par conséquent conduire au mariage des enfants comme moyen de garantir les moyens de subsistance.

Les préoccupations liées à la sexualité des filles sont également essentielles. Dans les contextes où la virginité avant le mariage est étroitement liée à l'honneur de la famille et à l'aptitude au mariage, l'insécurité financière accrue, l'anxiété et l'incertitude quant à l'avenir peuvent conduire les familles à marier leurs filles tôt afin d'éviter qu'elles ne s'engagent dans des relations sexuelles ou ne soient victimes de violences sexuelles. Dans certains contextes, les faibles opportunités économiques et l'insécurité financière peuvent conduire les filles à s'engager dans des relations sexuelles transactionnelles et par conséquent à tomber enceinte avant le mariage, ce qui est un facteur du mariage des enfants. Le risque accru de violence sexuelle peut également entraîner des grossesses et conduire au mariage des enfants. 
Figure 4. Comment la Covid-19 influence-t-elle le mariage des enfants ?

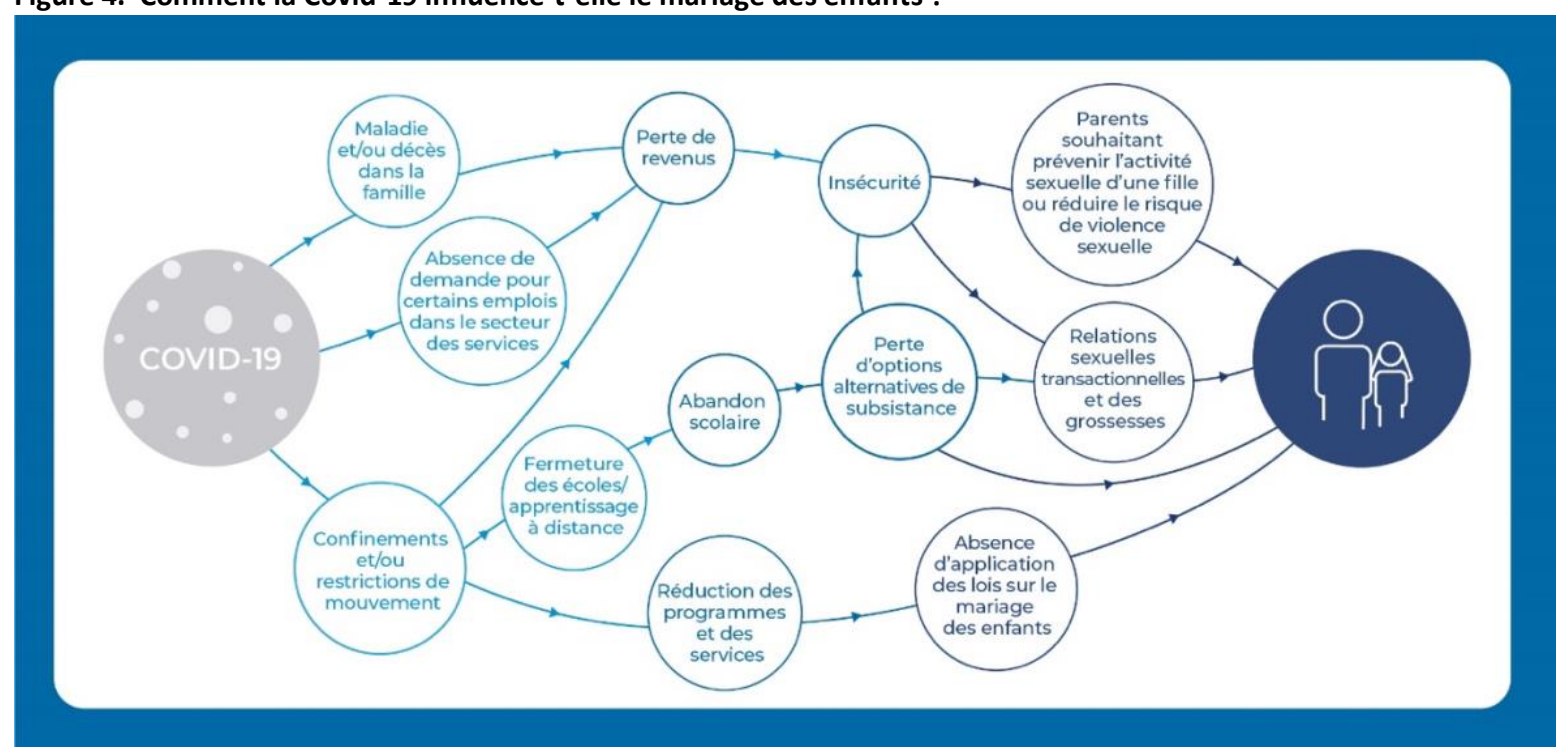

\section{Réactions à la Covid-19 dans les pays du programme MNCP}

Comme le montre le Tableau 5, chacun des pays concernés par le projet MNCP a pris les précautions nécessaires pour arrêter la propagation de la Covid-19, y compris la fermeture des écoles et, dans certains cas, des confinements extrêmes. Nous notons que les confinements liés à la Covid-19 et les adaptations ultérieures de la programmation varient en fonction des restrictions nationales et locales dans chaque contexte.

Tableau 5. Restrictions liées à la pandémie Covid-19, par pays

\begin{tabular}{|c|c|c|c|c|}
\hline Pays & $\begin{array}{l}\text { Premier cas de } \\
\text { Covid-19 } \\
\text { documenté }\end{array}$ & $\begin{array}{l}\text { Ordres de confinement ou de rester à la } \\
\text { maison }\end{array}$ & Restrictions de voyage & Fermeture des établissements scolaires \\
\hline Inde & 30 Jan 2020 & $\begin{array}{l}\text { Oui, confinement national : } \\
\text { Phase } 1 \text { ( } 25 \text { mars }-14 \text { avril) } \\
\text { Phase } 2 \text { ( } 15 \text { avril }-3 \text { mai) } \\
\text { Phase } 3 \text { ( } 4-17 \text { mai) } \\
\text { Phase } 4 \text { (18-31 mai) }\end{array}$ & $\begin{array}{l}\text { Oui, les visas de } \\
\text { tourisme sont } \\
\text { suspendus en mars } \\
2020 \text {; les } \\
\text { déplacements } \\
\text { internes sont limités }\end{array}$ & $\begin{array}{l}\text { Oui, mars- septembre } 2020 \\
\text { Après cette période, les états } \\
\text { pouvaient décider. En } \\
\text { novembre/décembre, quelques } \\
\text { écoles ont partiellement ouvert } \\
\text { pour faire passer les examens }\end{array}$ \\
\hline Mali & 25 Mar 2020 & $\begin{array}{l}\text { Oui, confinement en mars } 2020, \text { y } \\
\text { compris un couvre-feu jusqu'à } \\
\text { début mai. }\end{array}$ & $\begin{array}{l}\text { Oui, les vols sont } \\
\text { suspendus }\end{array}$ & $\begin{array}{l}\text { Oui, mars-juin } 2020 \text { (classes } \\
\text { d'examen ; Septembre pour les } \\
\text { autres) }\end{array}$ \\
\hline Malawi & 2 Apr 2020 & $\begin{array}{l}\text { Oui, le confinement national a } \\
\text { été ordonné mais bloqué par une } \\
\text { injonction ; les juridictions locales } \\
\text { pourraient émettre leur propre } \\
\text { confinement. }\end{array}$ & $\begin{array}{l}\text { Oui, les frontières } \\
\text { ont été fermées }\end{array}$ & Oui, mars-août 2020 \\
\hline Niger & 19 Mar 2020 & $\begin{array}{l}\text { Oui, les restrictions sont } \\
\text { appliquées de mars à mai ; les } \\
\text { mesures les plus strictes sont } \\
\text { appliquées à Niamey. }\end{array}$ & $\begin{array}{l}\text { Oui, toutes les } \\
\text { frontières terrestres } \\
\text { et aériennes ont été } \\
\text { fermées en mars } \\
2020 \text {; Niamey est } \\
\text { isolée du reste du } \\
\text { pays jusqu'au } 15 \\
\text { mai. }\end{array}$ & Oui, mars-juin 2020 \\
\hline
\end{tabular}


Impact de la Covid-19: Résultats

Les résultats de l'évaluation finale indiquent que la pandémie de Covid-19 exacerbe les indicateurs clés sur les voies hypothétiques vers le mariage des enfants. Les filles semblent souffrir de la Covid-19 et être exposées à un risque accru de mariage précoce. La Figure 5 présente les principaux résultats concernant l'impact de la pandémie sur la vie des filles à la fin de l'étude, en utilisant les données des enquêtes menées auprès des adolescents et des parents/adultes dans leurs ménages. 
Figure 5

\section{Impacts de la pandémie de Covid-19}

\section{FILLES}

SANTE ET BIEN-ETRE

Filles ayant déclaré se sentir plus déprimées

Filles ayant déclaré que les tensions dans le foyer ont augmenté

Filles s'inquiétant de tomber malade à cause du Coronavirus

Filles ayant des soucis d'argent

Filles ayant indiqué que l'accès aux services SDSR

est plus difficile pendant la pandémie

UTILISATION DU TEMPS

Filles passant plus de temps à faire des tâches ménagères

Filles passant plus de temps à s'occuper des enfants/ des personnes âgées

Filles passant plus de temps avec des amis

Filles passant plus de temps à la maison

FINANCES DES MÉNAGES ET INSÉCURITE

Filles ayant participé à une activité pour gagner de l'argent depuis le début de la pandémie

Filles ayant gagné moins d’argent qu'avant la pandémie

Filles estimant que dans leur quartier la violence a augmenté

PERTURBATION DE L'ÉDUCATION

Filles ayant fréquenté l'école juste avant la fermeture des établissements à cause de la Covid

Filles ayant déclaré être retournées à l'école depuis la réouverture des établissements

\section{PARENTS OU AUTRES MEMBRES ADULTES DU MENAGE}

REVENUS ET INSÉCURITÉ ALIMENTAIRE

Parents estimant que la Covid-19 a eu un impact négatif sur leurs finances

Parents ayant déclaré que le ménage a connu des pénuries alimentaires depuis le début de la pandémie

Parents ayant déclaré qu'au sein du ménage une fille a quitté le foyer depuis le début de la pandémie

ÉDUCATION DES FILLES

Parents ayant déclaré qu'au sein du ménage au moins une fille a pu continuer ses études pendant le confinement

Parents ayant déclaré qu'au sein du ménage au moins un garçon a pu continuer ses études pendant le confinement

UTILISATION DU TEMPS PAR LES FILLES

Les filles passent plus de temps à faire des tâches ménagères

Les filles passent plus de temps à s'occuper des enfants/personnes âgées
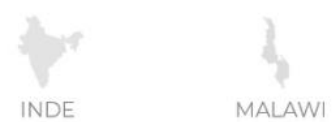

65.5

52.3
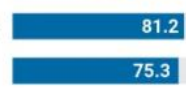

57.5

61.6

51.2

7.7

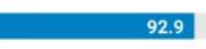

92.9

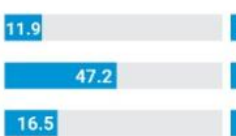

21.3
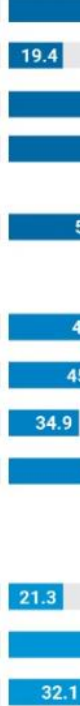

82.

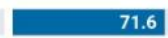

71.6

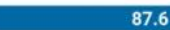

87.6

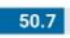

50.7

49.8

45.9

34.9

62.6

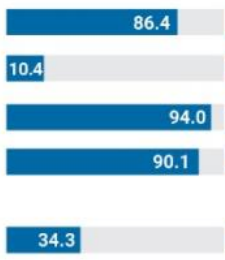

47.2

12.4

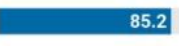

$\mathbf{8 1 . 8}$

29.8

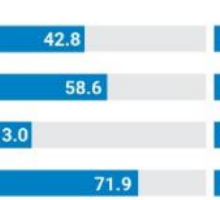

36.0

37.5

5.1

50.3
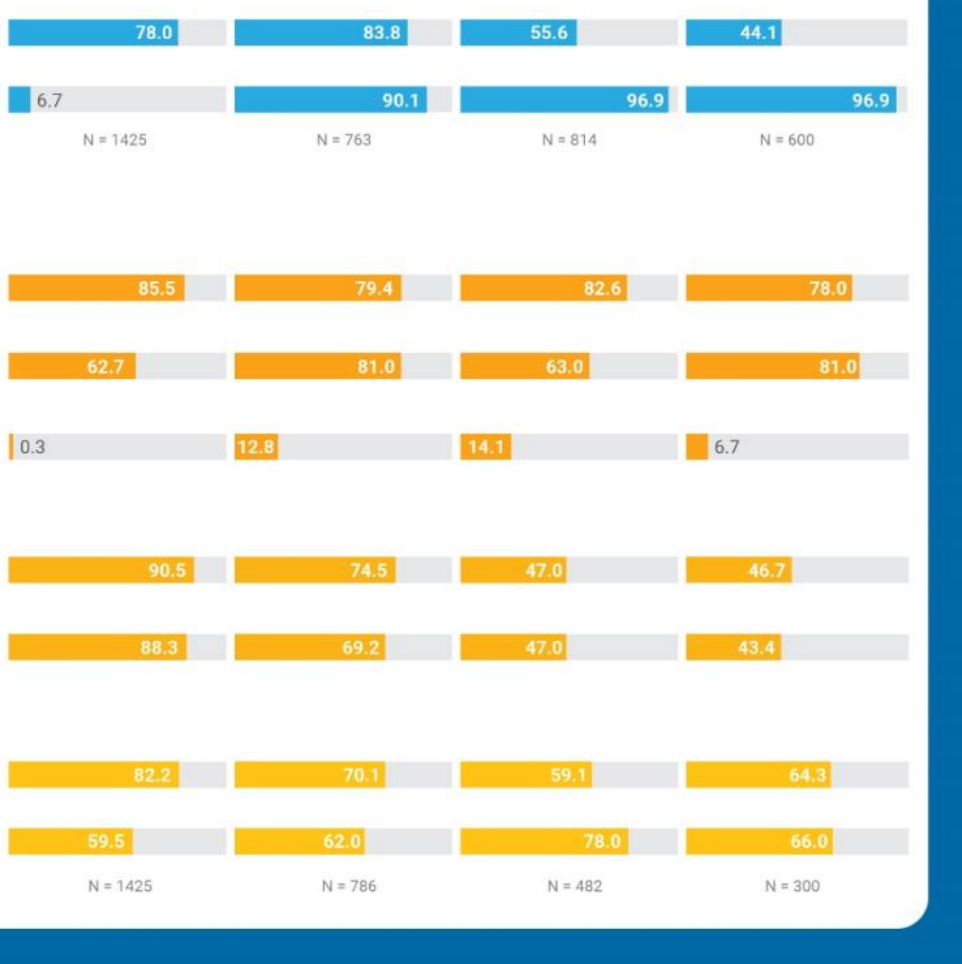


\section{Santé et bien-être}

Les résultats montrent que la pandémie de Covid-19 et les confinements et fermetures d'établissements scolaires qui ont suivi ont eu un effet sur la santé mentale des filles et sur l'accès aux services de santé qu'elles ont déclaré. Nous constatons que la majorité des filles (de $71,6 \%$ au Malawi à 94,0 \% au Mali) ont déclaré s'inquiéter de tomber malade à cause du coronavirus. Plus de la moitié des filles en Inde et au Malawi ont également déclaré avoir plus de difficultés à accéder aux services SDSR depuis le début de la pandémie. De nombreuses filles ont déclaré se sentir plus déprimées pendant la pandémie, de $47,2 \%$ au Niger à $86,4 \%$ au Mali.

\section{Finances et insécurité des ménages}

Nous constatons que l'augmentation de la pression financière sur les ménages depuis le début de la pandémie est communément signalée dans tous les pays, avec des effets importants sur la santé et le bien-être des ménages. La majorité des adultes interrogés dans tous les pays ont déclaré que la Covid-19 avait eu un impact négatif sur les finances de leur ménage et que plus de la moitié (et dans certains cas 4 sur 5 au Niger et au Malawi) des ménages déclaraient avoir connu des pénuries alimentaires depuis le début de la pandémie. De fortes proportions de filles (de 75,3\% en Inde à 90,1 \% au Mali) déclarent s'être inquiétées de l'argent pendant la pandémie. En Inde, où les mesures de confinement imposées par le gouvernement ont été les plus sévères, $52,3 \%$ de filles ont fait état de tensions accrues au sein de leur ménage. Certaines d'entre elles ont signalé que la violence dans leur quartier avait augmenté (d'un maximum de $32,0 \%$ de filles au Malawi à un minimum de $11,8 \%$ au Niger).

Au Mali et au Malawi, $14,1 \%$ et $13,2 \%$ des parents interrogés, respectivement, ont déclaré qu'une fille de 12 à 19 ans de leur foyer avait quitté leur village depuis le début de la pandémie. Nous notons que ces chiffres proviennent d'un échantillon de parents (ou d'un autre membre adulte du ménage) dont la fille adolescente était présente dans le foyer au moment de l'inscription des numéros de téléphone et a été invitée à participer à l'enquête sur les adolescents. Au Mali, les chercheurs ont constaté qu'une forte proportion de filles étaient absentes au moment de l'exercice d'énumération des numéros de téléphone, et que leurs ménages ont ensuite été remplacés dans l'échantillon par d'autres sélectionnés au hasard où la fille échantillonnée était présente. Les données de l'enquête auprès des adultes concernant les déplacements des adolescentes pendant la pandémie représentent donc une sous-estimation du véritable niveau de migration des filles au Mali. Les réponses sur les raisons du départ des filles (Figure 6) suggèrent que l'allègement de la pression économique sur

Figure 6. Raisons du départ du village pendant la pandémie (Malawi $n=101$, Mali $n=68$ )

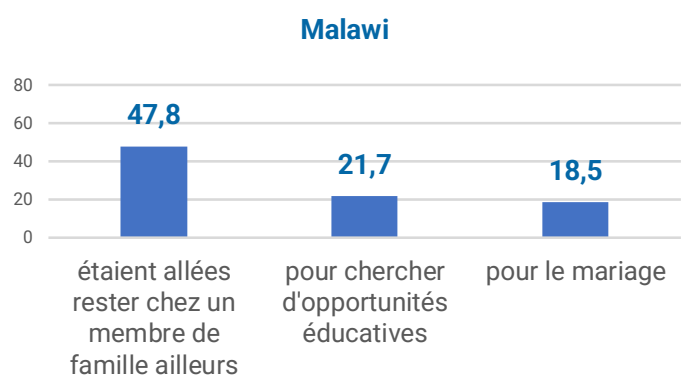

Mali

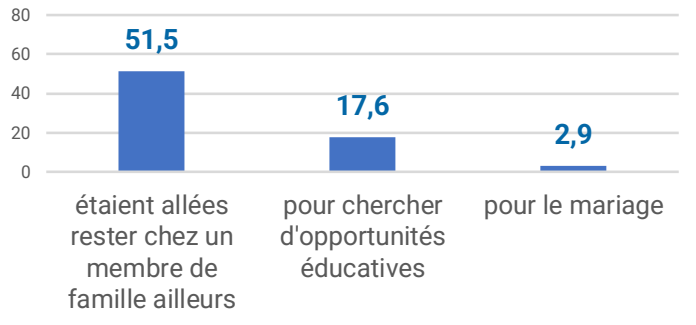


le ménage peut être un facteur clé du déplacement des filles en dehors de leurs villages, ce qui renforce les préoccupations concernant la vulnérabilité au mariage des enfants.

\section{Utilisation du temps}

Nous avons cherché à comprendre comment la pandémie de Covid-19 et la fermeture des établissements scolaires qui a suivi ont influencé la façon dont les filles passent leur temps et si les filles doivent assumer une plus grande charge de travail domestique au sein de leur foyer. En Inde, $61,6 \%$ des filles déclarent consacrer plus de temps aux tâches ménagères depuis le début de la pandémie. La majorité des filles ont déclaré avoir passé plus de temps à s'occuper des enfants ou des personnes âgées au Mali (58,6 \%) et en Inde (51,2\%).

\section{Perturbation de l'éducation}

Nous avons demandé aux adolescents et aux adultes interrogés dans quelle mesure les confinements de la Covid-19 avaient perturbé l'éducation des enfants de leur foyer. Nous avons constaté que dans les endroits où les écoles avaient rouvert leurs portes au moment de l'enquête, la majorité des filles inscrites dans ces écoles avant leur fermeture y étaient retournées. Bien que cela soit encourageant, moins de la moitié des ménages au Mali (47\%) et au Niger $(46,7 \%)$ ont déclaré qu'au moins une fille du foyer avait pu poursuivre ses études pendant la fermeture des écoles, alors qu'en Inde, 90,5\% des adultes interrogés ont déclaré qu'au moins une fille de leur foyer avait pu poursuivre ses études à distance. Nous n'avons pas trouvé de grandes différences entre les sexes, ce qui suggère que dans ces contextes, tant les filles que les garçons ont lutté pour poursuivre leurs études pendant la pandémie. Comme d'autres l'ont noté, le retard scolaire est fortement associé à l'abandon de l'école (Sunny et al 2017) et peut conduire à une grossesse précoce et à un mariage d'enfants (Glynn et al 2018).

\section{Implications pour l'interprétation des résultats de l'étude finale du MNCP}

Les résultats de l'étude finale du MNCP ont été recueillis après 7 à 9 mois de restrictions liées à la Covid-19, comme la fermeture des écoles et les restrictions de déplacement. Ces résultats ont probablement été influencés par l'expérience de ces restrictions. Bien que nous nous attendions à ce que les zones d'intervention et de comparaison connaissent des restrictions et des fermetures similaires, nous supposons que certaines zones ont pu bénéficier d'une plus grande

continuité du programme et peut-être d'expériences différentes telles que la scolarisation à distance. 


\section{CHAPITRE 4 \\ Résultats de l'étude finale : Inde}

\section{Contexte}

Malgré le recul enregistré ces dernières années, le mariage des enfants reste un problème important en Inde : on estime que 25,3\% des femmes âgées de 20 à 24 ans déclarent avoir été mariées avant l'âge de 18 ans, avec quelques variations géographiques notables (IIPS et ICF 2017). En 2015/6, les régions présentant le pourcentage le plus élevé de femmes âgées de 20 à 24 ans ayant déclaré s'être mariées avant l'âge de 18 ans étaient le Bihar (39,0\%) et le Jharkhand (37.0\%) (IIPS et ICF 2017). Nous avons constaté une baisse du mariage des enfants dans les données des enquêtes nationales sur la santé des familles ("National Family Health Survey » NFHS) de 2005-06 et 2015-16. Le Bihar, par exemple, a connu une baisse du nombre de mariages avant 18 ans chez les $20-24$ ans de $69,0 \%$ à $42,5 \%$. Alors que le mariage des enfants a diminué de manière significative dans certaines régions, il est resté relativement élevé dans d'autres, suggérant que le mariage des enfants est plus tenace dans les " points chauds " caractérisés par des facteurs géographiques, ethniques, religieux ou autres pouvant influencer la pratique.

Dans l'ensemble des recherches sur le mariage des enfants et ses facteurs de motivation en Inde, il est généralement admis que les principaux facteurs de motivation sont la pauvreté, les normes sexospécifiques, les investissements et les opportunités limités et inéquitables en matière d'éducation et de moyens de subsistance pour les filles, ainsi que les préoccupations concernant la sécurité sexuelle et la sécurité des filles. La pauvreté est généralement reconnue comme un facteur clé en Inde. Bien que des programmes réussis d'éducation aux moyens de subsistance et aux compétences de la vie courante soutiennent cette notion, davantage de preuves de causalité sont nécessaires. Au cours des trois dernières décennies, un certain nombre d'interventions visant à lutter contre le mariage des enfants en Inde ont connu un succès mitigé. Des examens systématiques, y compris un examen de 2021 par Malhotra et Elnakib, ont révélé qu'un certain nombre de programmes en Inde avaient réussi à réduire le mariage des enfants ou à le retarder, la plupart d'entre eux étant axés sur une approche axée sur les moyens de subsistance ou les compétences de la vie courante (Pande et al 2006 ; Jensen 2012) et un autre sur les informations SDSR (Daniel et Nanda 2012).

Comme l'indiquent les enquêtes nationales, le mariage des enfants a diminué en Inde au cours des dernières années. C'est également ce que nous constatons d'après les données du rapport à mi-parcours de ce projet : à mi-parcours, le mariage des enfants était en baisse en Inde dans la plupart des régions. Nous avons également constaté que le taux de scolarisation des filles était généralement élevé, en particulier dans les cohortes les plus jeunes, et que le programme avait connu un succès modeste au niveau de l'amélioration des connaissances sur la santé et les droits sexuels et reproductifs et de l'âge légal du mariage. 
Caractéristiques des échantillons

Le Tableau 6 ci-dessous indique les caractéristiques des échantillons en comparant les échantillons de base, à mi-parcours et finaux par zone d'intervention. D'une manière générale, nos échantillons étaient comparables pour tous les statuts d'intervention au niveau de l'étude de base.

Tableau 6. Caractéristiques des échantillons pour l'Inde, par état

\begin{tabular}{|c|c|c|c|c|c|c|}
\hline \multirow[b]{2}{*}{ Country } & \multicolumn{2}{|c|}{ Étude de base } & \multicolumn{2}{|c|}{ Étude à mi-parcours } & \multicolumn{2}{|c|}{ Étude finale } \\
\hline & Intervention & Comparison & Intervention & Comparison & Intervention & Comparison \\
\hline \multicolumn{7}{|l|}{ Bihar } \\
\hline Filles actuellement mariées & 16,0 & $11,0^{\star \star}$ & $6,2^{+++}$ & $4,5^{+++}$ & $7,6^{+++}$ & $10,3^{++}$ \\
\hline $\begin{array}{l}\text { Filles n'étant pas } \\
\text { scolarisées }\end{array}$ & 35,7 & $24,4^{\star \star \star}$ & $27,0^{++}$ & $24,7^{\star}$ & $20,0^{+++}$ & $20,2^{+}$ \\
\hline $\begin{array}{l}\text { Filles de } 15 \text { à } 19 \text { ans ayant } \\
\text { été enceintes (ayant déjà } \\
\text { été mariées) }\end{array}$ & 58,8 & 47,2 & 38,5 & $(10,0)^{\star \star}$ & $(66,7)$ & $(66,7)^{++}$ \\
\hline \multicolumn{7}{|l|}{ Jharkhand } \\
\hline Filles actuellement mariées & 26,9 & 25,9 & $18,6^{++}$ & $19,8^{+}$ & $9,3^{+++}$ & $19,3^{\star \star}$ \\
\hline $\begin{array}{l}\text { Filles n'étant pas } \\
\text { scolarisées }\end{array}$ & 45,2 & $40,9^{*}$ & $38,4^{++}$ & 37,4 & $19,3^{+++}$ & 25,0 \\
\hline $\begin{array}{l}\text { Filles de } 15 \text { à } 19 \text { ans ayant } \\
\text { été enceintes (ayant déjà } \\
\text { été mariées) }\end{array}$ & 57,7 & 56,1 & 57,6 & 54,4 & $(64,0)$ & 72,7 \\
\hline \multicolumn{7}{|l|}{ Odisha } \\
\hline Filles actuellement mariées & 8,9 & 6,0 & 7,8 & 7,1 & $0,0^{+++}$ & $2,2^{* *}$ \\
\hline $\begin{array}{l}\text { Filles n'étant pas } \\
\text { scolarisées }\end{array}$ & 36,3 & 45,3 & 40,4 & 43,3 & $15,0^{+++}$ & $26,7^{+++\star \star}$ \\
\hline $\begin{array}{l}\text { Filles de } 15 \text { à } 19 \text { ans ayant } \\
\text { été enceintes (ayant déjà } \\
\text { été mariées) }\end{array}$ & 52,9 & $(57,1)$ & 28,6 & 48,0 & - & $(20,0)$ \\
\hline
\end{tabular}

\begin{tabular}{|c|c|c|c|c|c|c|}
\hline \multicolumn{7}{|l|}{ Rajasthan } \\
\hline Filles actuellement mariées & 8,4 & 6,5 & 7,2 & $4,2^{*}$ & $2,1^{++}$ & $9,1^{\star *}$ \\
\hline $\begin{array}{l}\text { Filles n'étant pas } \\
\text { scolarisées }\end{array}$ & 35,7 & 33,3 & $26,5^{+++}$ & $22,8^{+++}$ & $16,8^{+++}$ & $15,7^{+++}$ \\
\hline $\begin{array}{l}\text { Filles ayant déjà été } \\
\text { enceintes (parmi les filles } \\
\text { mariées de } 15 \text { à } 19 \text { ans) }\end{array}$ & 14,7 & 15,3 & 5,8 & 4,7 & $(33,3)$ & $(40,0)^{+++}$ \\
\hline \multicolumn{7}{|c|}{ 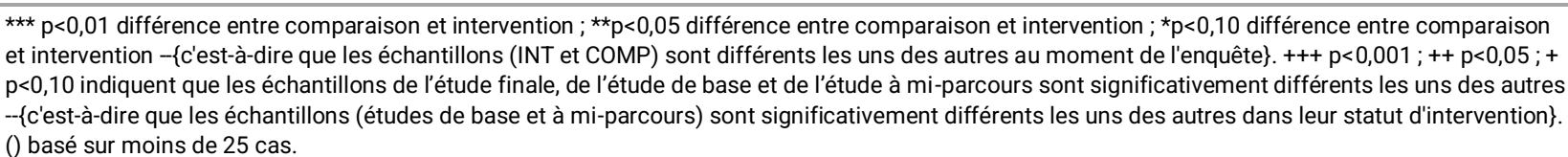 } \\
\hline
\end{tabular}




\section{Exposition au programme}

À la Figure 7 ci-dessous nous examinons si les personnes interrogées déclarent connaître le programme SRCM dans les zones d'intervention, de l'étude de base à l'étude finale. Nous constatons une augmentation de la connaissance du programme MNCP dans chaque état à l'exception de l'Odisha, et une connaissance encore plus accrue dans le Jharkhand. Nous constatons également une augmentation globale de la connaissance du programme de $20 \%$ (10 points de pourcentage) de l'étude de base à l'étude finale, avec près de $2 / 3$ des filles interrogées dans les zones d'intervention déclarant connaître le programme.
Figure 7. Proportion de personnes déclarant connaître le programme MTBA dans les zones d'intervention, de l'étude de base à l'étude finale

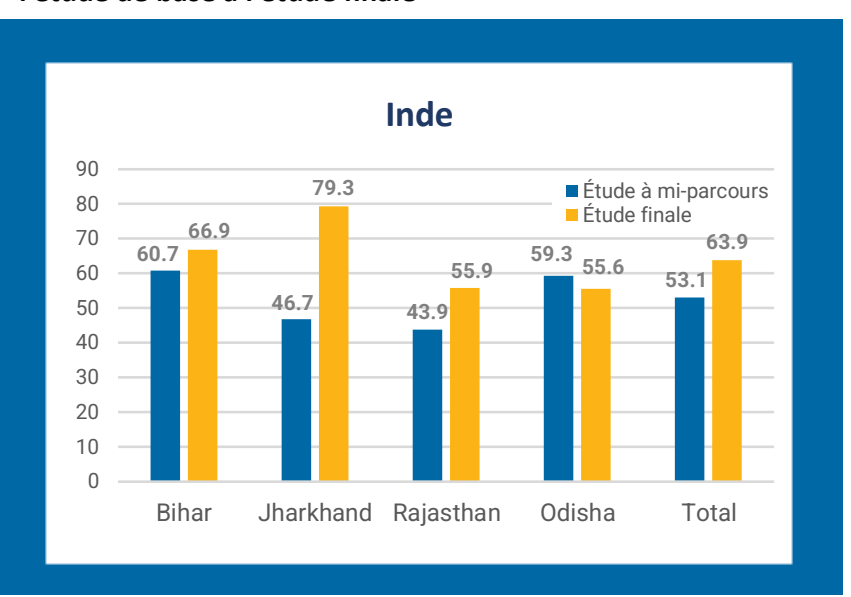

\section{Résultats}

Figure 8. Résumé des résultats, Inde

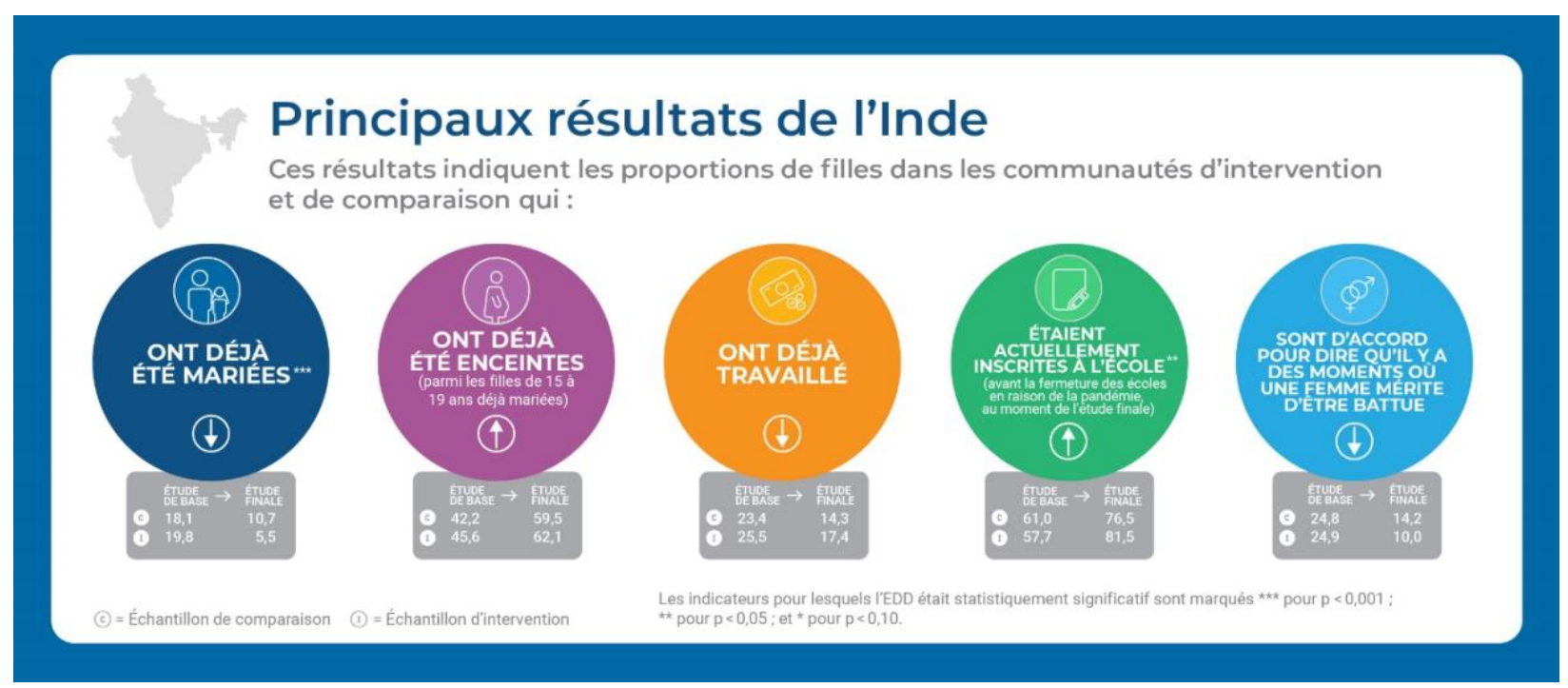

Dans le Tableau 7 ci-dessous, nous présentons les principaux résultats de l'analyse EDD sur les principaux indicateurs d'intérêt. Les flèches figurant à côté de INT et COM indiquent la direction du changement depuis l'étude de base à l'étude finale pour chaque indicateur dans les zones d'intervention et de comparaison, respectivement. Les résultats surlignés en bleu montrent les indicateurs pour lesquels l'EDD était significatif et les communautés d'intervention ont mieux performé que les communautés de comparaison du début à la fin de l'étude. 
Tableau 7. Résultats de l'étude finale, Inde

\begin{tabular}{|c|c|c|c|}
\hline $\begin{array}{l}\text { Domaine } \\
\text { d'impact }\end{array}$ & Indicateur & État & $\begin{array}{l}\text { Direction du changement de } \\
\text { l'étude de base à l'étude } \\
\text { finale }\end{array}$ \\
\hline \multirow{15}{*}{ Mariage } & \multirow{5}{*}{$\begin{array}{l}\text { Filles pouvant identifier correctement l'âge légal } \\
\text { du mariage }\end{array}$} & Tous les états & INT $\uparrow \mathrm{COM} \uparrow$ \\
\hline & & Bihar & INT $\uparrow \mathrm{COM} \uparrow$ \\
\hline & & Jharkhand & INT $\uparrow \mathrm{COM} \uparrow$ \\
\hline & & Odisha & INT $\uparrow \mathrm{COM} \uparrow$ \\
\hline & & Rajasthan & INT $\uparrow \mathrm{COM} \uparrow$ \\
\hline & \multirow[t]{5}{*}{ Filles n'ayant jamais été mariées } & Tous les états & INT $\downarrow$ COM $\downarrow$ \\
\hline & & Bihar & INT $\downarrow$ COM $\downarrow$ \\
\hline & & Jharkhand & INT $\downarrow$ COM $\downarrow$ \\
\hline & & Odisha & INT $\downarrow$ COM $\downarrow$ \\
\hline & & Rajasthan & INT $\downarrow$ COM $\uparrow$ \\
\hline & \multirow{5}{*}{$\begin{array}{l}\text { Filles pouvant identifier au moins trois } \\
\text { conséquences néfastes liées au mariage } \\
\text { précoce }\end{array}$} & Tous les états & INT $\uparrow \mathrm{COM} \uparrow$ \\
\hline & & Bihar & INT $\uparrow \mathrm{COM} \uparrow$ \\
\hline & & Jharkhand & INT $\uparrow \mathrm{COM} \uparrow$ \\
\hline & & Odisha & INT $\uparrow \mathrm{COM} \uparrow$ \\
\hline & & Rajasthan & INT $\uparrow \mathrm{COM} \uparrow$ \\
\hline \multirow{15}{*}{ Santé } & \multirow{5}{*}{$\begin{array}{l}\text { Filles ayant eu une grossesse (parmi les filles } \\
\text { qui n'ont jamais été mariées âgées de } 15 \text { à } 19 \\
\text { ans) }\end{array}$} & Tous les états & INT $\uparrow \mathrm{COM} \uparrow$ \\
\hline & & Bihar & INT $\uparrow \mathrm{COM} \uparrow$ \\
\hline & & Jharkhand & INT $\uparrow \mathrm{COM} \uparrow$ \\
\hline & & Odisha & INT - COM $\downarrow$ \\
\hline & & Rajasthan & INT $\uparrow \mathrm{COM} \uparrow$ \\
\hline & \multirow[t]{2}{*}{ Filles ayant des connaissances sur le VIH } & Tous les états & INT $\uparrow \mathbf{C O M} \uparrow$ \\
\hline & & Bihar & $\mathrm{INT} \uparrow \mathrm{COM} \uparrow$ \\
\hline & \multirow{8}{*}{$\begin{array}{l}\text { Filles indiquant que leur communauté dispose } \\
\text { d'un centre de santé adapté aux jeunes }\end{array}$} & Jharkhand & INT $\uparrow \mathbf{C O M} \uparrow$ \\
\hline & & Odisha & INT $\uparrow \mathrm{COM} \uparrow$ \\
\hline & & Rajasthan & INT $\uparrow \mathrm{COM} \uparrow$ \\
\hline & & Tous les états & INT $\uparrow \mathrm{COM} \uparrow$ \\
\hline & & Bihar & INT - COM - \\
\hline & & Jharkhand & INT - COM - \\
\hline & & Odisha & INT $\uparrow \mathrm{COM} \uparrow$ \\
\hline & & Rajasthan & $\mathrm{INT} \uparrow \mathrm{COM} \uparrow$ \\
\hline \multirow{10}{*}{$\begin{array}{l}\text { Moyens de } \\
\text { subsistance }\end{array}$} & \multirow[t]{5}{*}{ Filles ayant déjà travaillé pour un revenu } & Tous les états & INT $\downarrow$ COM $\downarrow$ \\
\hline & & Bihar & INT - COM $\uparrow$ \\
\hline & & Jharkhand & $\mathrm{INT} \downarrow \mathrm{COM} \downarrow$ \\
\hline & & Odisha & INT $\downarrow$ COM $\downarrow$ \\
\hline & & Rajasthan & $\mathrm{INT} \downarrow \mathrm{COM} \downarrow$ \\
\hline & \multirow[t]{5}{*}{ Filles travaillant actuellement pour un revenu } & Tous les états & $\mathrm{INT} \uparrow \mathrm{COM} \uparrow$ \\
\hline & & Bihar & INT $\uparrow \mathrm{COM} \uparrow$ \\
\hline & & Jharkhand & INT $\downarrow$ COM $\uparrow$ \\
\hline & & Odisha & INT $\uparrow \mathrm{COM}-$ \\
\hline & & Rajasthan & INT $\uparrow \mathrm{COM} \uparrow$ \\
\hline \multirow{2}{*}{ Éducation } & & Tous les états & INT $\uparrow$ COM - \\
\hline & & Bihar & INT $\uparrow \mathbf{C O M} \uparrow$ \\
\hline
\end{tabular}


Filles actuellement scolarisées (parmi celles qui l'ont été)

Filles ayant déjà été scolarisées

Niveau scolaire le plus élevé atteint

Filles sachant lire ou écrire

\begin{tabular}{|c|c|}
\hline Jharkhand & INT $\uparrow \mathrm{COM}-$ \\
\hline Rajasthan & INT $\uparrow \mathrm{COM} \uparrow$ \\
\hline Odisha & INT $\uparrow \mathrm{COM} \uparrow$ \\
\hline Tous les états & INT $\uparrow \mathrm{COM} \uparrow$ \\
\hline Bihar & INT $\uparrow \mathrm{COM} \uparrow$ \\
\hline Jharkhand & INT $\uparrow \mathrm{COM}-$ \\
\hline Rajasthan & INT $\uparrow \mathrm{COM} \uparrow$ \\
\hline Odisha & INT $\uparrow \mathrm{COM} \uparrow$ \\
\hline Tous les états & $\mathrm{INT} \uparrow \mathrm{COM} \uparrow$ \\
\hline Bihar & INT $\uparrow \mathrm{COM} \uparrow$ \\
\hline Jharkhand & INT $\uparrow \mathrm{COM} \uparrow$ \\
\hline Rajasthan & INT - COM $\uparrow$ \\
\hline Odisha & INT $\uparrow \mathrm{COM} \uparrow$ \\
\hline Tous les états & $\mathrm{INT} \uparrow \mathrm{COM} \uparrow$ \\
\hline Bihar & INT $\uparrow \mathrm{COM} \uparrow$ \\
\hline Jharkhand & INT $\uparrow \mathrm{COM} \uparrow$ \\
\hline Rajasthan & INT $\uparrow \mathrm{COM} \uparrow$ \\
\hline Odisha & INT $\uparrow \mathrm{COM} \uparrow$ \\
\hline Tous les états & INT $\uparrow \mathrm{COM} \uparrow$ \\
\hline Bihar & INT $\uparrow \mathrm{COM} \uparrow$ \\
\hline Jharkhand & INT $\uparrow \mathrm{COM} \uparrow$ \\
\hline Rajasthan & INT $\uparrow \mathrm{COM} \uparrow$ \\
\hline Odisha & INT $\uparrow \mathrm{COM} \uparrow$ \\
\hline Tous les états & INT $\uparrow \mathrm{COM} \uparrow$ \\
\hline Bihar & INT $\uparrow \mathrm{COM} \uparrow$ \\
\hline Jharkhand & INT $\uparrow \mathrm{COM} \uparrow$ \\
\hline Rajasthan & INT $\uparrow \mathrm{COM} \uparrow$ \\
\hline Odisha & INT $\uparrow \mathrm{COM} \uparrow$ \\
\hline Tous les états & INT $\downarrow$ COM $\downarrow$ \\
\hline Bihar & INT $\downarrow$ COM $\downarrow$ \\
\hline Jharkhand & $\mathrm{INT} \downarrow \mathrm{COM} \downarrow$ \\
\hline Rajasthan & INT $\downarrow$ COM - \\
\hline Odisha & $\mathrm{INT} \downarrow \mathrm{COM} \downarrow$ \\
\hline Tous les états & INT $\uparrow \mathrm{COM} \uparrow$ \\
\hline Bihar & INT $\uparrow \mathrm{COM} \uparrow$ \\
\hline Jharkhand & INT $\uparrow \mathrm{COM} \uparrow$ \\
\hline Rajasthan & INT - COM $\uparrow$ \\
\hline Odisha & INT $\uparrow \mathrm{COM} \uparrow$ \\
\hline Tous les états & INT - COM - \\
\hline Bihar & INT $\uparrow \mathrm{COM}-$ \\
\hline Jharkhand & $\mathrm{INT} \uparrow \mathrm{COM} \uparrow$ \\
\hline Rajasthan & INT - COM $\downarrow$ \\
\hline Odisha & $\mathrm{INT} \downarrow \mathrm{COM} \downarrow$ \\
\hline Tous les états & INT $\uparrow \mathrm{COM}-$ \\
\hline Bihar & INT $\uparrow \mathrm{COM} \downarrow$ \\
\hline Jharkhand & INT $\uparrow$ COM - \\
\hline Rajasthan & INT $\uparrow \mathrm{COM}-$ \\
\hline Odisha & INT $\uparrow \mathrm{COM}-$ \\
\hline
\end{tabular}

Filles étant d'accord sur le fait qu'une femme ait le droit de divorcer

Filles étant d'accord sur le fait qu'une femme puisse ne pas être d'accord avec ses parents (beaux-parents) sur les décisions qui la concernent

Filles déclarant faire partie d'un club ou d'un groupe

\section{Vie sociale}

\section{Attitudes équitables entre les}

Note : Les résultats surlignés en bleu montrent les indicateurs pour lesquels l'EDD était significatif et les communautés d'intervention ont obtenu de meilleurs résultats que les communautés de comparaison. 
Nous constatons que le programme MNCP en Inde a réussi à augmenter la connaissance de l'âge légal du mariage et à diminuer la proportion de filles actuellement mariées. Les filles vivant dans les zones d'intervention étaient moins susceptibles d'être mariées à la fin de l'étude que les filles des zones de comparaison, bien que les deux zones aient enregistré un déclin du mariage des enfants au cours de la période d'évaluation.

Nous constatons également des améliorations de certains indicateurs de santé en Inde. La connaissance du VIH a plus que doublé dans les communautés d'intervention en Inde (de 22,4\% à $50,2 \%$ ), avec des augmentations plus modestes dans les communautés de comparaison. Dans les communautés d'intervention de Jharkhand, la connaissance du VIH est passée de seulement $6,2 \%$ en début d'étude à $37,3 \%$ en fin d'étude.

Le programme MNCP a réussi à augmenter le nombre d'inscriptions à l'école, les zones d'intervention du MNCP montrant une augmentation des inscriptions alors que les zones de comparaison n'ont connu aucun changement. L'intervention a également eu un impact positif sur la proportion de filles ayant déjà été scolarisées dans le Jharkhand et dans l'échantillon global de l'Inde. Le programme MNCP a moins bien réussi à augmenter la proportion de filles ayant déjà travaillé (aucun changement) et à améliorer les attitudes équitables entre les hommes et les femmes (quelques changements significatifs, mais seulement dans certains états).

Cependant, nous constatons un effet positif significatif du programme MNCP sur la participation à un club. En effet, dans tous les états, et dans l'ensemble les filles des zones d'intervention déclarent faire partie d'un club ou d'un groupe par rapport aux filles des zones de comparaison. Dans les zones d'intervention du programme MNCP, la participation à un club a augmenté alors qu'elle a diminué dans les zones de comparaison.

Ces résultats sont encourageants et suggèrent que le programme MNCP réussit à retarder le mariage, peut-être par le biais de la création d'actifs sociaux, notamment la participation à un club ou à un groupe, et par l'amélioration de l'éducation et le maintien des filles à l'école. Nous constatons un impact sur la réduction du mariage des enfants dans tous les états (à l'exception du Bihar), bien que ces états aient commencé avec des divers niveaux de mariage des enfants : Le Rajasthan (8,4\%) et l'Odisha $(8,9 \%)$ avaient un taux de mariage d'enfants relativement bas au départ, comparé au Jharkhand (26,9\%). Dans l'ensemble, le mariage des enfants dans les zones d'intervention a diminué de $14,5 \%$ à $4,5 \%$, soit une baisse globale de $69 \%$ (les zones de comparaison ont diminué de $22 \%$ ). La proportion de filles déclarant faire partie d'un club ou d'un groupe s'est également améliorée de façon spectaculaire entre l'étude de base $(2,7 \%$ dans les zones d'intervention) et l'étude finale $(49,8 \%)$, soit une multiplication de près de 20 de cet indicateur. 


\section{Résultats de l'étude finale : Malawi}

\section{Contexte}

Le mariage des enfants au Malawi a diminué, passant de plus de la moitié en 1992 (54,9\% des femmes âgées de 20 à 24 ans déclarent avoir été mariées avant l'âge de 18 ans, selon l'EDS de 1992) à 42,1 \% (NSO [Malawi] et ICF 2017), mais reste parmi les proportions les plus élevées d'Afrique subsaharienne. Des recherches qualitatives (Steinhaus et al., 2019 ; Ansell et al., 2018) indiquent que la grossesse est un facteur important pour comprendre pourquoi le mariage des enfants reste élevé au Malawi. Des recherches antérieures ont montré que l'activité sexuelle prémaritale est courante et que les premiers rapports sexuels ont lieu tôt au Malawi. Biddlecom et ses collègues (2008) ont constaté que parmi les filles âgées de 18 à 19 ans ayant terminé l'école primaire, $48,1 \%$ ont déclaré avoir déjà eu des rapports sexuels et $27,0 \%$ ont déclaré avoir eu des rapports sexuels avant le mariage alors qu'elles étaient encore scolarisées.

Une grande partie des nombreuses recherches effectuées sur les interventions visant à retarder le mariage au Malawi est incluse dans les examens systématiques de ce qui fonctionne pour lutter contre le mariage des enfants. Les interventions incluses dans ces examens se sont principalement concentrées sur les incitations économiques, telles que les transferts directs en espèces, mises en œuvre pour retarder le mariage, suggérant que les incitations économiques directement fournies aux filles et aux familles peuvent permettre de retarder le mariage, au moins jusqu'à ce que les paiements cessent (Baird et al., 2016). Dans le cadre des recherches qualitatives menées pour ce projet, nous avons constaté que le mariage est une stratégie de subsistance et que trouver un partenaire capable de subvenir à ses besoins peut être la meilleure option économique pour les filles (Population Council, 2019). Malgré des taux élevés de scolarisation primaire pour les filles, peu d'entre elles déclarent suivre un enseignement supérieur et l'écart entre les revenus des femmes et ceux des hommes reste important (Kim, 2020).

\section{Note méthodologique}

Comme nous l'avons indiqué dans le rapport de mi-parcours (Melnikas et al., 2019), nous avons inclus des analyses supplémentaires, outre l'analyse en intention de traiter, afin de tenir compte des problèmes de contamination et de fidélité à l'intervention mis en lumière au début de 2018. Étant donné que l'unité de mise en œuvre du programme était les villages des chefs de groupe (GHV), une unité pouvant changer au fil du temps, et que l'unité d'analyse de la recherche était la zone de dénombrement (EA), nous avons eu quelques problèmes pour que les EA reçoivent l'intervention. Cette analyse, que nous avons dénommée "telle que mise en œuvre », a été présentée en même temps que l'analyse en intention de traiter. Dans cette analyse, nous présentons les résultats «tels qu'ils ont été mis en œuvre » afin de tenir compte de la manière 
dont les communautés ont reçu le MNCP, mais nous n'ajustons pas les covariables supplémentaires. Nous notons qu'en 2020, un groupe supplémentaire (EA) a été déterminé comme étant contaminé et classé comme intervention dans cette analyse (précédemment classé comme comparaison à mi-parcours). Nous avons également dû abandonner trois groupes en raison du manque de filles éligibles dans la liste des données de mi-parcours disponibles en phase d'étude finale.

\section{Caractéristiques de l'échantillon}

Le Tableau 8 ci-dessous présente quelques données démographiques clés par statut d'intervention pour les trois études. Dans l'ensemble, nous constatons que les échantillons sont généralement comparables dans les zones d'intervention et de comparaison. De l'étude de base à l'étude finale, nous constatons des baisses dans les résultats clés, y compris la grossesse et le mariage, mais ces baisses ont eu lieu dans les zones d'intervention et de comparaison.

Tableau 8. Caractéristiques de l'échantillon, Malawi

\begin{tabular}{|c|c|c|c|c|c|c|}
\hline & \multicolumn{2}{|c|}{ Étude de base } & \multicolumn{2}{|c|}{ Étude à mi-parcours } & \multicolumn{2}{|c|}{ Étude finale } \\
\hline & Intervention & Comparaison & Intervention & Comparaison & Intervention & Comparaison \\
\hline Âge (moyen) & 14,8 & 15,0 & 15,0 & 14,9 & $14,4^{+++}$ & $14,6^{+}$ \\
\hline $\begin{array}{l}\text { Filles ayant déjà été } \\
\text { mariées }\end{array}$ & 16,2 & 15,1 & 11,0 & 8,1 & $6,3^{+++}$ & $5,2^{+++}$ \\
\hline $\begin{array}{l}\text { Filles n'ayant jamais été } \\
\text { scolarisées }\end{array}$ & 4,1 & 0,9 & 4,0 & 4,4 & 2,2 & $1,9^{++}$ \\
\hline Filles non scolarisées & 35,0 & 31,4 & 33,4 & 28,7 & $17,8^{+++}$ & $12,8^{+++*}$ \\
\hline $\begin{array}{l}\text { Filles ne sachant ni lire ni } \\
\text { écrire }\end{array}$ & 32,3 & 21,4 & 23,7 & 18,1 & $14,4^{+++}$ & $13,2^{++}$ \\
\hline $\begin{array}{l}\text { Filles ayant déjà été } \\
\text { enceintes (âgées de } 15 \text { à } \\
19 \text { ans) }\end{array}$ & 22,8 & 24,7 & 25,6 & 25,8 & $17,0+^{+}$ & $14,8^{++}$ \\
\hline
\end{tabular}

\section{Exposition au programme}

La connaissance du programme MNCP dans les zones « telles que mises en œuvre » a augmenté de manière significative entre l'étude à mi-parcours et l'étude finale $(6,5 \%$ des villages où le programme MTBA a été mis en œuvre ont déclaré connaître le programme à mi-parcours de l'étude et cette proportion est passée à $64,5 \%$ en phase d'étude finale. Cependant, les données d'exposition au programme montrent également une connaissance élevée du programme MNCP dans les zones de non-intervention $(63,4 \%$ des répondants de la communauté de comparaison 
déclarent connaître le MNCP à la fin de l'étude. Parmi les personnes des zones d'intervention qui connaissaient le MTBA à la fin de l'étude, 45,1\% ont déclaré avoir participé à une activité MTBA.
Figure 9. Proportion de personnes déclarant connaître le programme MTBA dans les zones d'intervention, de l'étude de base à l'étude finale

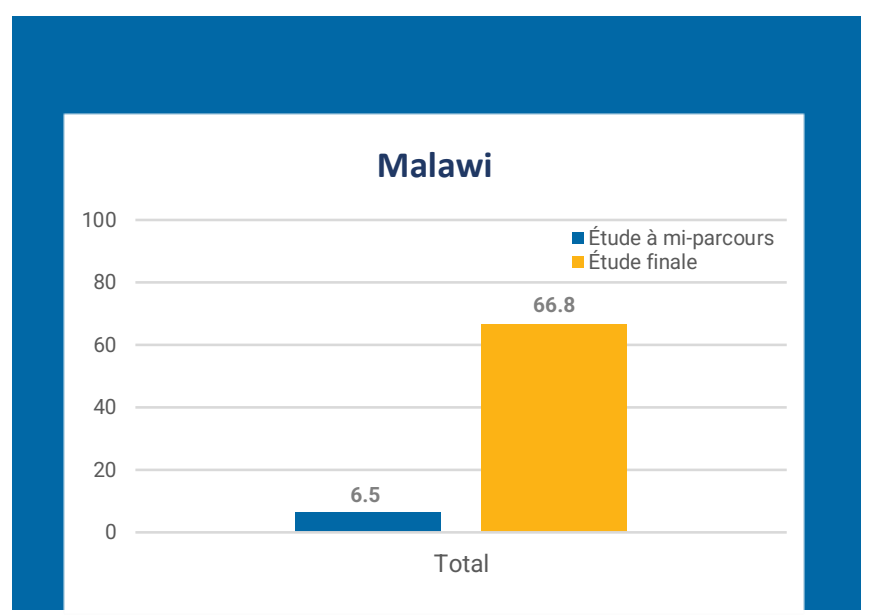




\section{Résultats}

Figure 10. Résumé des résultats, Malawi

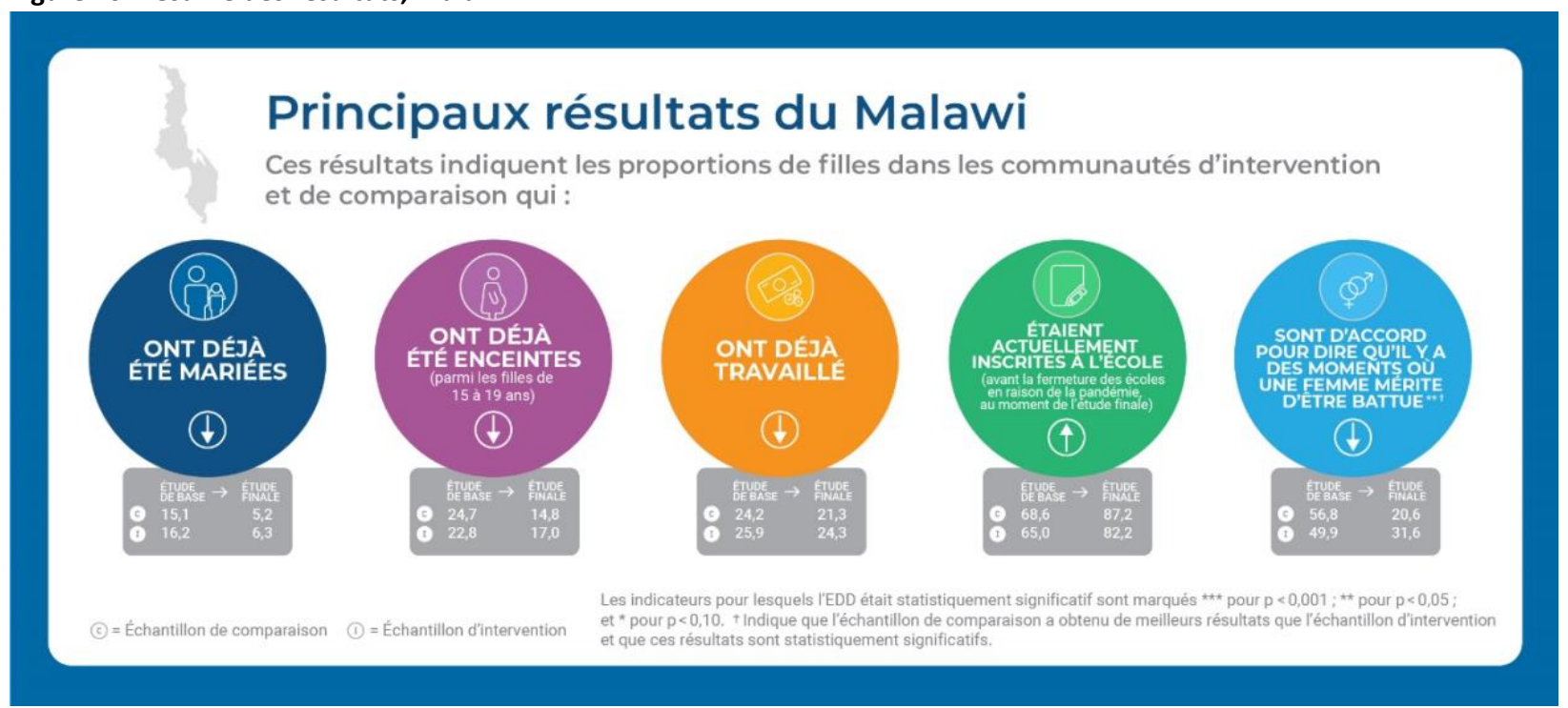

Le Tableau 9 ci-dessous présente les résultats de l'évaluation d'impact pour le Malawi. Les flèches qui suivent INT et COM montrent la direction du changement par indicateur de l'étude de base à l'étude finale dans les zones d'intervention et de comparaison, respectivement. Les indicateurs surlignés en bleu montrent les résultats pour lesquels l'EDD était significatif et les zones d'intervention ont obtenu de meilleurs résultats que les zones de comparaison. Les indicateurs surlignés en jaune montrent les résultats pour lesquels l'EDD était significatif, mais des améliorations plus importantes ont été observées dans les zones de comparaison, par rapport aux zones d'intervention.

Tableau 9. Résultats de l'étude finale, Malawi

\begin{tabular}{|c|c|c|}
\hline Domaine d'impact & Indicateur & $\begin{array}{l}\text { Direction du changement de } \\
\text { l'étude de base à l'étude finale }\end{array}$ \\
\hline \multirow{4}{*}{ Mariage } & Filles ayant déjà été mariées & $\mathrm{INT} \downarrow \mathrm{COM} \downarrow$ \\
\hline & Filles pouvant identifier correctement l'âge légal du mariage & INT $\uparrow$ COM - \\
\hline & $\begin{array}{l}\text { Filles pouvant identifier au moins trois conséquences néfastes } \\
\text { liées au mariage précoce }\end{array}$ & INT $\uparrow \mathrm{COM} \uparrow$ \\
\hline & Age moyen au mariage & INT $\uparrow \mathrm{COM}-$ \\
\hline \multirow{3}{*}{ Santé } & $\begin{array}{l}\text { Filles ayant déjà eu une grossesse (parmi les filles de } 15 \text { à } 19 \\
\text { ans) }\end{array}$ & INT $\downarrow$ COM $\downarrow$ \\
\hline & Filles ayant des connaissances sur le VIH & INT $\uparrow \mathrm{COM} \uparrow$ \\
\hline & $\begin{array}{l}\text { Indique que leur communauté dispose d'un centre de santé } \\
\text { adapté aux jeunes }\end{array}$ & INT $\uparrow \mathrm{COM} \uparrow$ \\
\hline \multirow{3}{*}{$\begin{array}{l}\text { Moyens de } \\
\text { subsistance }\end{array}$} & Échelle de connaissance des contraceptifs (méthodes modernes) & INT $\uparrow \mathrm{COM} \uparrow$ \\
\hline & Filles ayant déjà travaillé pour un revenu & INT - COM - \\
\hline & Filles travaillant actuellement pour un revenu & INT $\uparrow \mathrm{COM} \uparrow$ \\
\hline Éducation & Filles actuellement scolarisées (parmi celles qui l’ont été) & $\mathrm{INT} \uparrow \mathrm{COM} \uparrow$ \\
\hline
\end{tabular}




\begin{tabular}{|c|c|c|}
\hline & Filles ayant déjà été scolarisées & INT $\uparrow \mathrm{COM} \uparrow$ \\
\hline & Nombre moyen d'années d'études accomplies & INT $\uparrow$ COM - \\
\hline & Filles ne sachant ni lire ni écrire & INT $\downarrow$ COM $\downarrow$ \\
\hline & $\begin{array}{l}\text { Filles étant d'accord sur le fait que les garçons aient le droit de } \\
\text { refuser un mariage arrangé }\end{array}$ & INT $\uparrow \mathrm{COM}-$ \\
\hline & $\begin{array}{l}\text { Filles étant d'accord sur le fait que les filles aient le droit de } \\
\text { refuser un mariage arrangé }\end{array}$ & INT $\uparrow \mathrm{COM} \mathrm{-}$ \\
\hline $\begin{array}{l}\text { équitables entre } \\
\text { les sexes }\end{array}$ & $\begin{array}{l}\text { Filles étant d'accord sur le fait qu'une femme mérite parfois d'être } \\
\text { battue }\end{array}$ & INT $\downarrow \mathrm{COM} \downarrow$ \\
\hline & Filles étant d'accord sur le fait qu'une femme a le droit de divorcer & INT $\uparrow \mathrm{COM} \mathrm{-}$ \\
\hline & $\begin{array}{l}\text { Filles étant d'accord sur le fait qu'une femme puisse ne pas être } \\
\text { d'accord avec ses parents (beaux-parents) sur les décisions qui la } \\
\text { concernent }\end{array}$ & INT $\uparrow \mathrm{COM} \uparrow$ \\
\hline Vie sociale & Filles déclarant faire partie d'un club ou d’un groupe & INT $\downarrow$ COM $\downarrow$ \\
\hline
\end{tabular}

En ce qui concerne le principal indicateur lié au mariage, nous constatons des baisses dans les zones d'intervention et de comparaison. Bien que cela soit encourageant, nous ne voyons pas de différence significative dans les zones d'intervention, suggérant que le déclin du mariage des enfants au Malawi au cours de la période du projet peut être dû à des facteurs plus importants au-delà du projet. En plus du déclin du mariage des enfants, la connaissance des effets néfastes du mariage a augmenté dans les zones d'intervention et de comparaison. La connaissance de l'âge légal du mariage au Malawi a augmenté de manière significative dans les zones d'intervention, montrant l'impact du programme (44,5 \% à 59,5\% dans les zones d'intervention, contre $45,6 \%$ à $51,1 \%$ dans les zones de comparaison).

Nous observons des tendances similaires sur les résultats de santé : les filles déclarant avoir déjà été enceintes ont diminué dans les zones d'intervention et de comparaison entre l'étude de base et l'étude finale, mais il n'y a pas de différence significative. Nous constatons également une forte augmentation de la proportion de filles indiquant que leur communauté dispose d'un centre de santé adapté aux jeunes (de 4,3 \% à 59,3\% dans les zones d'intervention et de 3,8 \% à $52,7 \%$ dans les zones de comparaison). Cependant, comme ces fortes augmentations se sont produites dans les zones d'intervention et de comparaison, nous ne voyons pas de différences significatives attribuables au programme MNCP.

Dans les domaines des moyens de subsistance et de l'éducation, des améliorations significatives ont été constatées dans les zones d'intervention et de comparaison. La proportion de filles, par exemple, ayant déclaré travailler actuellement pour gagner de l'argent (parmi celles ayant déjà travaillé) a augmenté d'environ 26 points de pourcentage (soit une multiplication par trois) dans les zones d'intervention entre le début et la fin de l'étude $(13,1 \%$ à $39,0 \%)$. L'augmentation a été similaire dans les zones de comparaison (15,6\% à 47,3\%). Les filles ont également gagné du terrain dans le taux de scolarisation actuel, passant de $65 \%$ à $83,2 \%$ (intervention) et de $68,6 \%$ à $87,9 \%$ (comparaison). Le programme MNCP a réussi à démontrer son impact sur l'augmentation de la proportion de filles ayant déjà fréquenté l'école, l'augmentation étant plus importante dans les zones d'intervention que dans les zones de comparaison $(p<0,10)$. Le MNCP a également 
réussi à augmenter le nombre moyen d'années d'éducation, l'augmentation étant de 0,5 année dans les zones d'intervention (3,9 à 4,5 années d'éducation), tandis que l'éducation des filles des zones de comparaison ont perdu du terrain (4,7 à 4,6 années d'éducation). Le MNCP a également montré des effets significatifs sur l'alphabétisation : la proportion de filles ne sachant ni lire ni écrire a chuté de 32,3 à $14,4 \%$ dans les zones d'intervention et de 21,4 à $13,2 \%(p<0,10)$ dans les zones de comparaison.

Depuis le début de l'étude, nous constatons des résultats positifs en ce qui concerne les attitudes équitables entre hommes et femmes : davantage de filles sont d'accord pour dire que les filles ont le droit de refuser un mariage arrangé et que les filles ont le droit de divorcer. Ces indicateurs montrent une tendance positive dans les zones d'intervention, tandis que la plupart sont restés les mêmes dans les zones de comparaison, bien que ces résultats ne soient pas statistiquement significatifs. Cependant, l'énoncé selon lequel il y a des moments où une femme mérite d'être battue a connu une forte baisse ( 49,9 à $31,6 \%$ dans les zones d'intervention et 56,8 à $20,6 \%$ dans les zones de comparaison), les zones de comparaison ayant une baisse plus importante.

De manière surprenante, nous constatons que moins de filles dans les zones d'intervention et de comparaison déclarent être membres d'un club à la fin de l'étude par rapport à l'étude de base (baisse de $24,6 \%$ à $23,5 \%$ de l'étude de base à l'étude finale dans les zones d'intervention et de $26,7 \%$ à $16,3 \%$ dans les zones de comparaison), bien que les filles notamment des zones d'intervention aient rapporté une baisse moindre de l'adhésion à un club $(p=0,109)$. Cela peut être dû en partie à l'influence des restrictions liées à la Covid-19, notamment la fermeture des établissements scolaires et les restrictions de mobilité et de rassemblement qui ont limité la capacité des clubs à se réunir régulièrement en 2020. 


\section{Résultats de l'étude finale : Mali}

\section{Contexte}

Avec 52,6 \% des filles mariées avant l'âge de 18 ans et 18,3 \% des filles mariées avant l'âge de 15 ans (INSTAT CPS/SS- DS-PF et ICF 2019), le Mali a la cinquième prévalence la plus élevée du mariage des enfants dans le monde (UNICEF, 2018). Le Mali inclut quelques variations géographiques : le mariage des enfants est surtout concentré dans les régions rurales du sudouest du pays qui enregistre les plus fortes proportions de femmes de 20 à 24 ans déclarant s'être mariées avant l'âge de 18 ans à Kayes (75,2 \%), Tombouctou (70,0 \%) et Sikasso (58,8 \%). (INSTAT CPS/SS- DS-PF et ICF 2019). A Ségou, deuxième région où le programme MNCP a été mis en œuvre, la proportion de femmes de 20 à 24 ans déclarant s'être mariées avant l'âge de 18 ans est de 52,8 \%. De 2001 à 2018, la proportion de femmes âgées de 20 à 24 ans qui étaient mariées avant l'âge de 18 ans est passée de 70,6 \% à 53,7 \% et la proportion de femmes mariées avant l'âge de 15 ans est passée de 24,6 \% à 15,9\%. Cependant, la proportion d'adolescentes ayant commencé à avoir des enfants n'a pratiquement pas changé au cours de la dernière décennie (35,5\% en 2006 à 36,0 \% en 2018). On estime que 46,2\% des filles ont donné naissance à un enfant avant l'âge de 18 ans et 13,6 \% avant l'âge de 15 ans.

Comme l'ont montré les recherches qualitatives menées dans le cadre de ce projet (Engebretsen et al 2020) et d'autres (Grosz-Ngate 2000 ; Hertrich et Lesclingand 2013), la migration est courante chez les adolescentes au Mali et est influencée à la fois par la préparation au mariage et par la volonté d'éviter un mariage précoce non désiré. La migration saisonnière pour des opportunités de travail agricole est également courante dans certaines régions - à mi-parcours, nous avons constaté que la migration saisonnière chez les adolescentes était courante, en particulier à Ségou (Melnikas et al., 2019). Le nombre élevé de mariages d'enfants au Mali peut être lié au manque d'investissement dans l'éducation des filles. Au Mali, l'accès des filles à l'éducation est limité et environ la moitié des femmes âgées de 20 à 24 ans (52,0\%) déclarent ne pas avoir reçu d'éducation formelle (INSTAT CPS/SS- DS-PF et ICF 2019). Parmi les enfants en âge de fréquenter l'école primaire, $68,9 \%$ déclarent avoir fréquenté l'école primaire (INSTAT CPS/SS- DS-PF et ICF 2019), ce qui est supérieur au pourcentage du Niger $(56,4)$ mais bien inférieur au pourcentage des pays voisins, la Côte d'Ivoire (94,9\%) et la Guinée (89.4) (DHS stat compiler).

\section{Caractéristiques de l'échantillon}

Dans le Tableau 10 ci-dessous, nous examinons les principales données démographiques des échantillons de l'enquête. Nous constatons que le mariage a diminué entre le début et la fin de l'enquête dans tous les échantillons, tout comme la proportion de filles déclarant ne jamais être allées à l'école et être enceintes. À la fin de l'enquête, un peu plus de filles ont déclaré ne pas être scolarisées, ce qui peut être dû en partie au moment de l'enquête et à la fermeture des écoles. 
Tableau 10. Caractéristiques de l'échantillon, Mali

\begin{tabular}{|c|c|c|c|c|c|c|}
\hline & \multicolumn{2}{|c|}{ Étude de base } & \multicolumn{2}{|c|}{ Étude à mi-parcours } & \multicolumn{2}{|c|}{ Étude finale } \\
\hline & Intervention & Comparaison & Intervention & Comparaison & Intervention & Comparaison \\
\hline Âge (moyen) & 15,2 & 15,3 & 15,1 & $14,9^{++}$ & 15,2 & 15,2 \\
\hline $\begin{array}{l}\text { Filles ayant déjà été } \\
\text { mariées }\end{array}$ & 11,9 & 17,1 & $6,9^{+++}$ & $8,9^{+++}$ & 7,2 & $6,9^{+++}$ \\
\hline $\begin{array}{l}\text { Filles n'ayant jamais été } \\
\text { scolarisées }\end{array}$ & 32,7 & $40,8^{\star \star}$ & 30,6 & $48,5^{\star \star \star+}$ & $21,4^{+++}$ & $33,5^{\star \star \star++}$ \\
\hline Filles non scolarisées & 33,1 & 38,5 & 27,3 & 25,8 & $41,0^{++}$ & $48,3^{+++\star \star}$ \\
\hline $\begin{array}{l}\text { Filles ne sachant ni lire ni } \\
\text { écrire }\end{array}$ & 44,3 & 55,4 & 41,0 & 57,4 & $30,7^{+++}$ & $39,4^{+++\star \star}$ \\
\hline $\begin{array}{l}\text { Filles ayant déjà été } \\
\text { enceintes (âgées de } 15 \text { à } \\
19 \text { ans) }\end{array}$ & 20,1 & 24,4 & 17,9 & 22,1 & 13,4 & 17,5 \\
\hline
\end{tabular}

$\star \star \star * 00,01$ différence entre comparaison et intervention ; ${ }^{\star \star} p<0,05$ différence entre comparaison et intervention (c'est-à-dire que les échantillons d'intervention et de comparaison sont différents l'un de l'autre au moment de l'enquête).

$+++p<0,01$ différence entre l'étude de base, l'étude à mi-parcours et l'étude finale; $++p<0,05$ différence entre l'étude de base, l'étude à mi-parcours et l'étude finale ; + p<0,10 différence entre l'étude de base, l'étude à mi-parcours et l'étude finale (c'est-à-dire que les échantillons de l'étude de base et de l'étude à mi-parcours sont significativement différents les uns des autres dans leur statut d'intervention).

\section{Exposition au programme}

La proportion de filles dans les zones d'intervention déclarant connaître le programme MNCP a augmenté de $21,3 \%$ à mi-parcours à $36,2 \%$ à la fin du programme, ce qui montre que le programme a réussi à accroître la reconnaissance du nom et à atteindre davantage de filles dans ces communautés depuis l'étude à mi-parcours menée en 2018 (Figure 13).
Figure 11. Proportion de personnes déclarant connaître le programme MTBA dans les zones d'intervention, de l'étude de base à l'étude finale, Mali

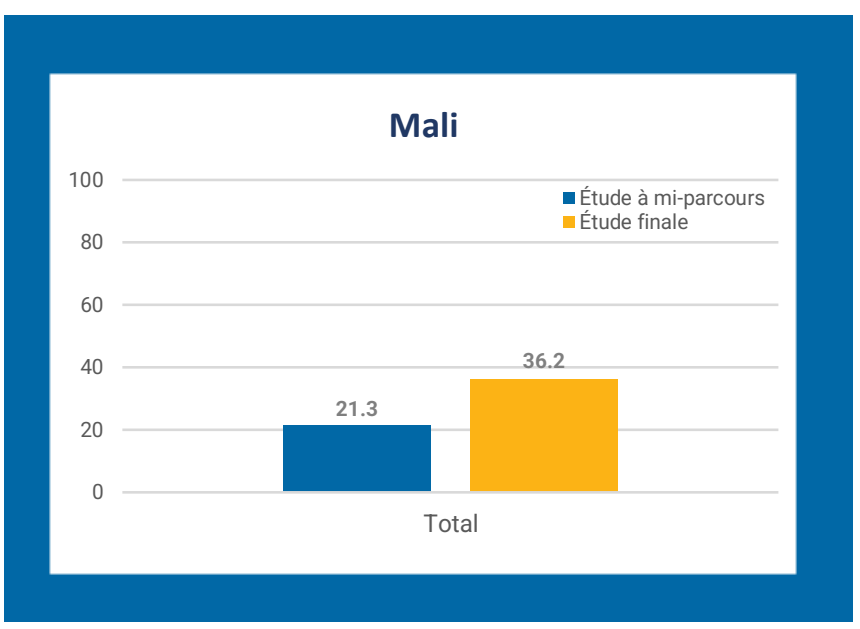




\section{Résultats}

Figure 12. Résumé des résultats, Mali

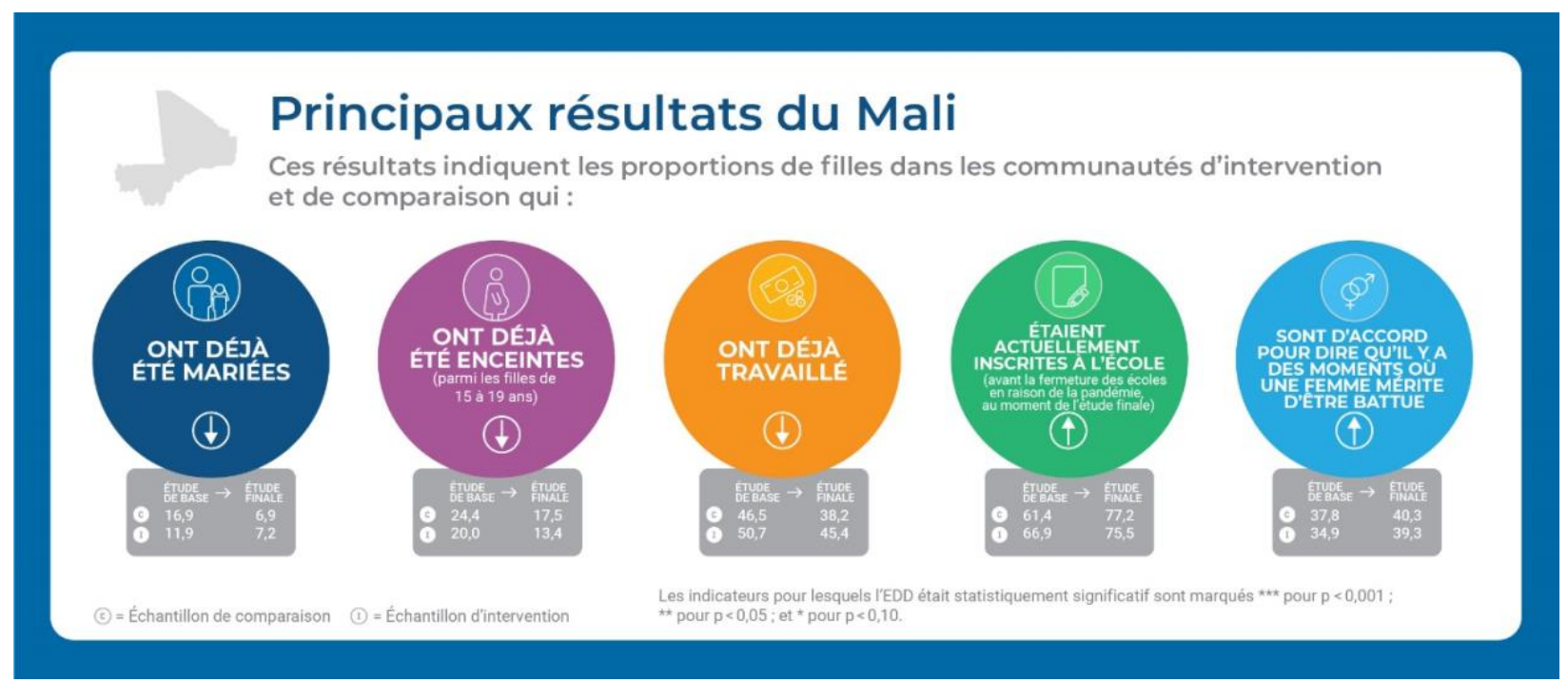

Le Tableau 11 ci-dessous présente les résultats de l'évaluation d'impact pour le Mali. Les flèches à côté de INT et COM montrent la direction du changement pour chaque indicateur entre l'étude de base et l'étude finale dans les zones d'intervention et les zones de comparaison, respectivement. Les indicateurs surlignés en bleu représentent l'impact du programme (EDD significatifs avec des zones d'intervention plus performantes que les zones de comparaison). Les indicateurs surlignés en jaune représentent les résultats pour lesquels l'EDD était significatif mais les zones de comparaison ont dépassé les zones d'intervention.

Tableau 11. Résultats de l'étude finale, Mali

\begin{tabular}{|c|c|c|}
\hline Domaine d'impact & Indicateur & $\begin{array}{l}\text { Direction du changement de } \\
\text { l'étude de base à l'étude } \\
\text { finale }\end{array}$ \\
\hline \multirow{4}{*}{ Mariage } & Filles ayant déjà été mariées & INT $\downarrow$ COM $\downarrow$ \\
\hline & Filles pouvant identifier correctement l'âge légal du mariage & INT - COM - \\
\hline & $\begin{array}{l}\text { Filles pouvant identifier au moins trois conséquences néfastes liées } \\
\text { au mariage précoce }\end{array}$ & INT $\uparrow \mathrm{COM} \uparrow$ \\
\hline & Âge moyen au mariage & INT $\uparrow$ COM - \\
\hline \multirow{5}{*}{ Santé } & Filles ayant déjà eu une grossesse (parmi les filles de 15 à 19 ans) & $\mathrm{INT} \downarrow \mathrm{COM} \downarrow$ \\
\hline & Filles ayant des connaissances sur le VIH & INT - COM - \\
\hline & Filles sachant que l'utilisation d'un préservatif protège du VIH & INT $\uparrow \mathrm{COM} \uparrow$ \\
\hline & $\begin{array}{l}\text { Filles indiquant que leur communauté dispose d'un centre de santé } \\
\text { adapté aux jeunes }\end{array}$ & INT $\uparrow \mathrm{COM} \uparrow$ \\
\hline & Échelle de connaissance des contraceptifs (méthodes modernes) & INT $\uparrow \mathrm{COM} \uparrow$ \\
\hline \multirow{2}{*}{$\begin{array}{l}\text { Moyens de } \\
\text { subsistance }\end{array}$} & Filles ayant déjà travaillé pour un revenu & INT - COM $\downarrow$ \\
\hline & Filles travaillant actuellement pour un revenu & $\mathrm{INT} \downarrow \mathrm{COM} \downarrow$ \\
\hline Éducation & Filles actuellement scolarisées (parmi celles qui l'ont déjà été) & INT $\downarrow$ COM $\downarrow$ \\
\hline
\end{tabular}




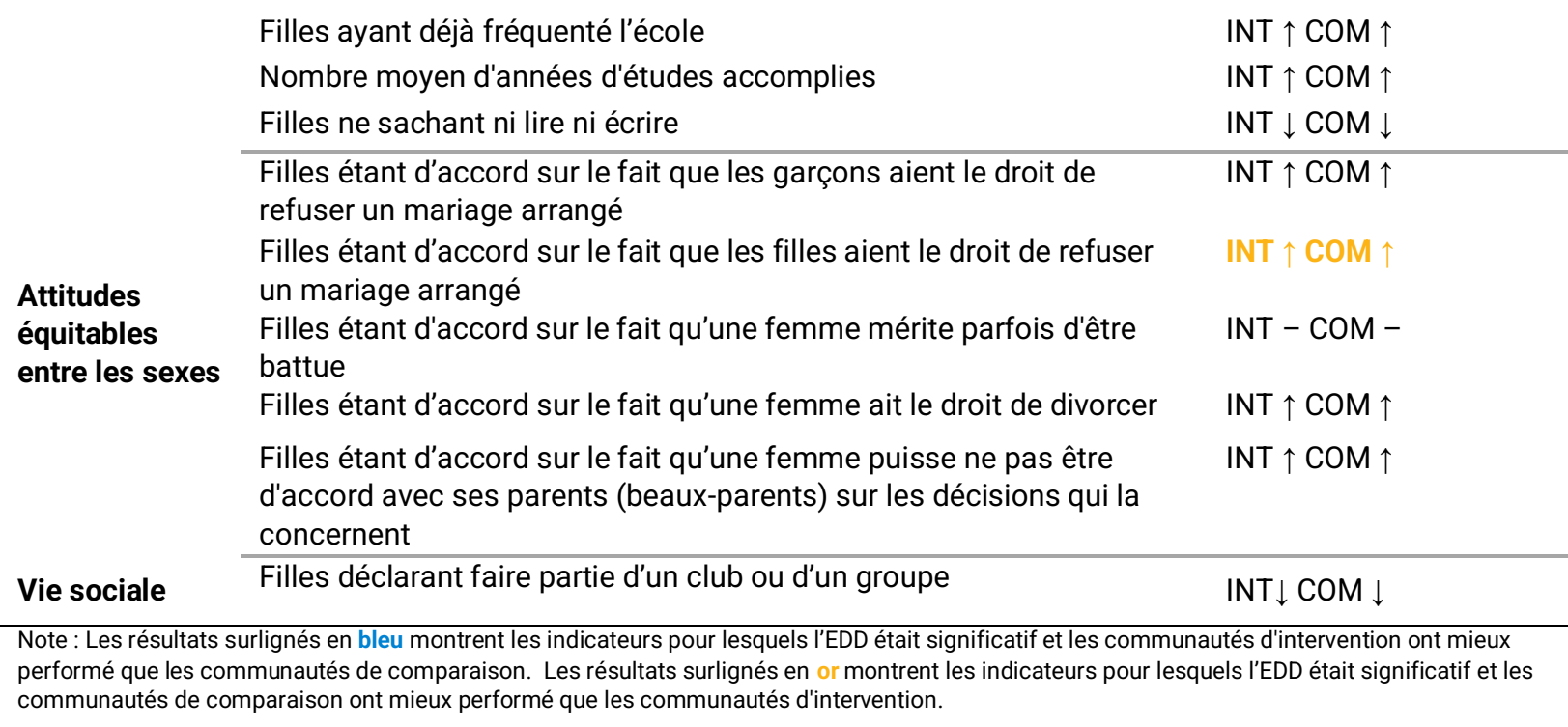

Alors que la proportion de filles de l'échantillon ayant déclaré avoir été mariées a diminué de $11,9 \%$ à $7,2 \%$ dans les communautés d'intervention et de $16,9 \%$ à $6,9 \%$ dans les communautés de comparaison entre l'étude de base et l'étude finale, nous n'avons pas constaté de différences significatives sur cet indicateur. Nous observons cette même tendance dans la proportion de filles capables d'identifier trois effets négatifs du mariage précoce (de $13,6 \%$ à 22,1 \% dans les communautés d'intervention et de $9,5 \%$ à $16,8 \%$ dans les communautés de comparaison). Sur la base de ces deux indicateurs, nous concluons que les baisses observées sont probablement le résultat d'influences plus importantes que le seul programme MNCP. Malgré l'absence d'impact sur la proportion de filles mariées, parmi celles qui se marient, nous constatons un impact significatif sur l'âge du mariage des filles dans les zones d'intervention (de 15,9 ans à 16,8 ans), suggérant que le programme a une influence sur le report du mariage après les années d'adolescence pour les filles qui se marient tôt. Malgré l'absence d'impact sur la proportion des filles mariées, parmi celles qui se marient, nous constatons un impact significatif sur l'âge moyen des filles lors du mariage dans les zones d'intervention (de 15,9 à 16,8 ans), ce qui suggère que le programme a une influence sur le retard du mariage à des années ultérieurs pour les adolescentes qui se marient tôt.

Malheureusement, nous ne constatons pas d'impact du programme sur les principaux indicateurs de santé au Mali. Nous constatons une diminution de la proportion de filles de l'échantillon déclarant avoir déjà été enceintes dans les zones d'intervention (de 20,0\% à 13,4\%) et dans les zones de comparaison (de $24,4 \%$ à $17,5 \%$ ) ainsi qu'une augmentation de la proportion de filles sachant que l'utilisation d'un préservatif protège contre le $\mathrm{VIH}$ (de $44,6 \%$ à $75,1 \%$ dans les villages d'intervention et de $39,0 \%$ à $71,0 \%$ dans la zone de comparaison) et de la proportion de filles déclarant que leur communauté dispose d'un centre de santé adapté aux jeunes (de 8,5 \% à 60,4 \% dans les villages d'intervention et de $8,2 \%$ à 63,4 \% dans les villages de comparaison). L'augmentation spectaculaire de cet indicateur peut refléter les efforts du programme pour accroître la sensibilisation et la disponibilité de ces ressources, qui peuvent se trouver dans plusieurs zones et expliquer l'augmentation dans les zones de comparaison. 
Aucun changement significatif n'a été observé dans la proportion de filles connaissant le VIH ou ayant une connaissance correcte des méthodes de contraception modernes.

Dans les villages d'intervention et de comparaison, nous avons observé une baisse de la proportion de filles déclarant travailler actuellement pour gagner leur vie (de 50,9 \% à 45,3\% dans les communautés d'intervention et de $46,6 \%$ à 38,1\% dans les communautés de comparaison) et de la proportion de filles déclarant aller à l'école (de $66,9 \%$ à $59,4 \%$ dans les communautés d'intervention et de $61,4 \%$ à $51,7 \%$ dans les communautés de comparaison). Cette tendance peut être due à l'impact de la Covid-19, car nous savons que les écoles ont été fermées pendant une partie de l'année 2020 au Mali et nous pouvons supposer que les moyens de subsistance ont également été limités.

Cependant, des tendances positives ont été observées dans les domaines de l'éducation autres que la scolarisation actuelle. La proportion de filles déclarant être déjà allé à l'école a augmenté dans les deux échantillons (de $67,3 \%$ à 78,6 \% dans les communautés d'intervention et de 59,2\% à $66,5 \%$ dans les communautés de comparaison), tout comme le nombre moyen d'années de scolarité accomplies (de 4,2 à 4,7 ans dans les communautés d'intervention et de 3,6 à 4,1 ans dans les communautés de comparaison). La proportion de filles ne sachant ni lire ni écrire a en outre diminué partout (de 44,3\% à 30,7\% dans les villages d'intervention et de $55,4 \%$ à $39,4 \%$ dans les communautés de comparaison).

La proportion de filles étant d'accord sur le fait que les filles ont le droit de refuser un mariage arrangé a augmenté dans les villages d'intervention et de comparaison avec un EDD significatif. Cependant, l'augmentation la plus significative a été observée dans les zones de comparaison (de $22,8 \%$ à $54,46 \%$ contre de $32,3 \%$ à $53,2 \%$ dans les zones de comparaison). Nous pensons que cette tendance pourrait être en partie due à des événements communautaires importants axés sur la sensibilisation aux droits des filles et aux méfaits du mariage des enfants auxquels certains individus ont pu être exposés dans les zones de comparaison.

En examinant les attitudes équitables entre les sexes, dans les échantillons d'intervention et de comparaison, nous avons constaté une augmentation de la proportion de filles étant d'accord pour dire qu'une femme doit avoir le droit de divorcer (de 33,8 \% à 43,7 \% dans les communautés d'intervention et de $34,1 \%$ à $45,6 \%$ dans les communautés de comparaison) et qu'une fille peut être en désaccord avec ses parents (ou son mari si elle est mariée) sur les décisions qui affectent sa vie (de $24,1 \%$ à $34,0 \%$ dans les communautés d'intervention et de $24,2 \%$ à $33,5 \%$ dans les villages de comparaison).

La proportion de filles déclarant faire partie d'un club ou d'un groupe a diminué de manière similaire dans l'échantillon d'intervention (de $61,6 \%$ à 14,9\%) et dans l'échantillon de comparaison (de 58,3\% à 13,4 \%) entre l'étude de base et l'étude finale. La fermeture des écoles et autres restrictions liées à la pandémie de Covid-19 ont probablement joué un rôle dans ce déclin observé, car de nombreux clubs se trouvaient dans les écoles et l'augmentation de la charge de travail à la maison et les inquiétudes liées au virus ont pu empêcher les filles de se rassembler ailleurs. 


\section{Résultats de l'étude finale : Niger}

\section{Contexte}

Malheureusement, en raison des problèmes liés à qualité des données, le programme EDS n'a pas publié les résultats des enquêtes menées en 2017 au Niger, de sorte que les enquêtes disponibles les plus récentes datent de 2012. Selon l'EDS de 2012, le Niger a le taux de prévalence des mariages d'enfants le plus élevé au monde avec $77 \%$ des filles et des femmes (25-49 ans) mariées avant l'âge de 18 ans et 28 \% mariées avant l'âge de 15 ans. Les données sur la prévalence indiquent une baisse de 7 points de pourcentage (de 83,5 à 66,3 ) de la proportion de femmes de 20 à 24 ans mariées à l'âge de 18 ans sur la période 1992-2012. Sur cette même période, la proportion de femmes mariées à 15 ans a diminué d'environ 22 points de pourcentage (de 50,3 à 28,0) (Institut national de la statistique (INS) et ICF International, 2013).

La pratique du mariage des enfants est profondément ancrée au Niger et est restée relativement stagnante au fil du temps par rapport aux pays voisins (Fenn et al., 2015). L'âge légal du mariage pour les filles au Niger est de 15 ans. Grâce à de récentes recherches menées dans le cadre de la MTBA, nous avons constaté qu'au Niger les filles ont une attitude plutôt positive à l'égard du mariage précoce, car elles considèrent le mariage comme un rite de passage essentiel pour gagner le respect et l'admiration de leurs pairs et de la communauté dans son ensemble. Ces attitudes semblent être influencées à la fois par des opportunités éducatives et économiques très limitées et par une forte socialisation favorisant l'obéissance et la pratique culturelle du mariage saisonnier des filles dans les groupes de pairs (Saul et al., 2021). Ainsi, dans ce contexte, nombre de filles peuvent penser que le fait de ne pas saisir l'occasion qui leur est offerte de se marier serait plus préjudiciable à leur avenir que de se marier tôt.

D'autres facteurs influencent la forte proportion de mariages d'enfants au Niger. La polygamie est une pratique courante au Niger, avec environ $36 \%$ des femmes mariées actuellement dans des unions polygames. Les recherches ont montré que la polygamie est associée au mariage des enfants et à des différences d'âge plus importantes entre les conjoints et peut avoir une incidence négative sur le pouvoir de décision des femmes au sein des ménages (Wetheridge \& Antonowicz, 2014). Le Niger est également un pays considérablement exposé aux risques climatiques, notamment à la sécheresse et, par conséquent, à l'insécurité alimentaire. L'insécurité et les conflits récents ont également augmenté les risques de mariage d'enfants. Des recherches antérieures ont démontré que des facteurs tels que les conflits et les déplacements susceptibles d'aggraver l'incertitude financière des familles peuvent entraîner une augmentation des mariages d'enfants (Alston et al., 2014 ; Mourtada et al., 2017). 


\section{Caractéristiques de l'échantillon}

Dans le Tableau 12, nous présentons les caractéristiques de l'échantillon pour chacune des trois études. Nous notons que la proportion de filles déclarant avoir été mariées a diminué entre l'étude de base et l'étude finale dans les zones d'intervention et de comparaison, et que la proportion de filles déclarant n'avoir jamais été à l'école a diminué. Le nombre de grossesses chez les filles âgées de 15 à 19 ans a augmenté dans les zones d'intervention et de comparaison au cours des trois études (de 12,7\% pour l'étude de base à 19,6\% pour l'étude finale dans les zones de comparaison; de $14,6 \%$ à $17,7 \%$ dans les zones d'intervention).

Tableau 12. Caractéristiques de l'échantillon, Niger

\begin{tabular}{|c|c|c|c|c|c|c|}
\hline & \multicolumn{2}{|c|}{ Étude de base } & \multicolumn{2}{|c|}{ Étude à mi-parcours } & \multicolumn{2}{|c|}{ Étude finale } \\
\hline & Intervention & Comparaison & Intervention & Comparaison & Intervention & Comparaison \\
\hline Âge (moyen) & 15,2 & 15,3 & 15,1 & $14,9^{++}$ & 15,2 & 15,2 \\
\hline $\begin{array}{l}\text { Filles ayant déjà été } \\
\text { mariées }\end{array}$ & 25,7 & 37,7 & $18,2^{++}$ & $26,3^{\star \star \star+++}$ & $16,0^{+++}$ & $15,9^{+++}$ \\
\hline $\begin{array}{l}\text { Filles n'ayant jamais } \\
\text { fréquenté l'école }\end{array}$ & 29,7 & $46,5^{\star \star \star}$ & 25,0 & $45,1^{* \star \star}$ & 23,9 & $31,2^{+++\star \star}$ \\
\hline $\begin{array}{l}\text { Filles n'ayant jamais } \\
\text { été scolarisées }\end{array}$ & $27,5^{\star \star \star}$ & 49,0 & $29,0^{\star \star \star}$ & 51,0 & $36,7^{+}$ & $43,0 *$ \\
\hline $\begin{array}{l}\text { Filles ne sachant ni } \\
\text { lire ni écrire }\end{array}$ & 45,0 & $64,0^{\star \star \star}$ & 44,7 & $63,5^{\star \star \star}$ & $22,9^{+++}$ & $31,9^{+++\star \star}$ \\
\hline $\begin{array}{l}\text { Filles ayant déjà été } \\
\text { enceintes (âgées de } \\
15 \text { à } 19 \text { ans) }\end{array}$ & 14,6 & 12,7 & 10,7 & 19,0 & 17,7 & 19,6 \\
\hline
\end{tabular}

\section{Exposition au programme}

L'exposition au programme, mesurée par la proportion de filles dans les zones d'intervention déclarant connaître le programme MNCP, a considérablement augmenté entre l'étude à miparcours (2018) et l'étude finale (2020), une augmentation de près de 40 points de pourcentage (25,3 \% à 63,0 \%) (Figure 13). Cela suggère que le programme a réussi à améliorer la reconnaissance du nom et la sensibilisation au MNCP dans les zones d'intervention.
Figure 13. Proportion de personnes déclarant connaître le programme MTBA dans les zones d'intervention, de l'étude à mi-parcours à l'étude finale, Niger

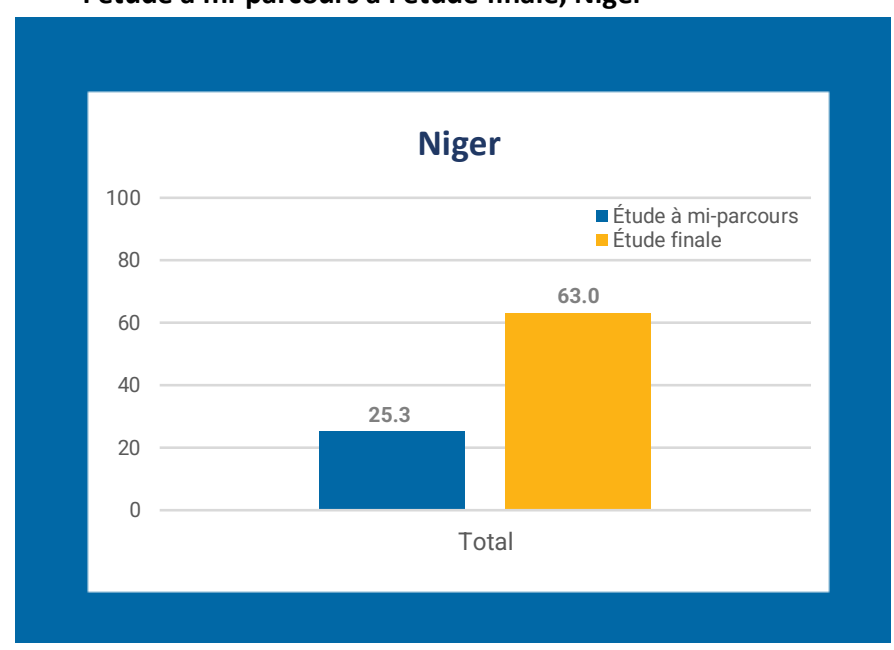




\section{Résultats}

Figure 14. Résumé des résultats, Niger

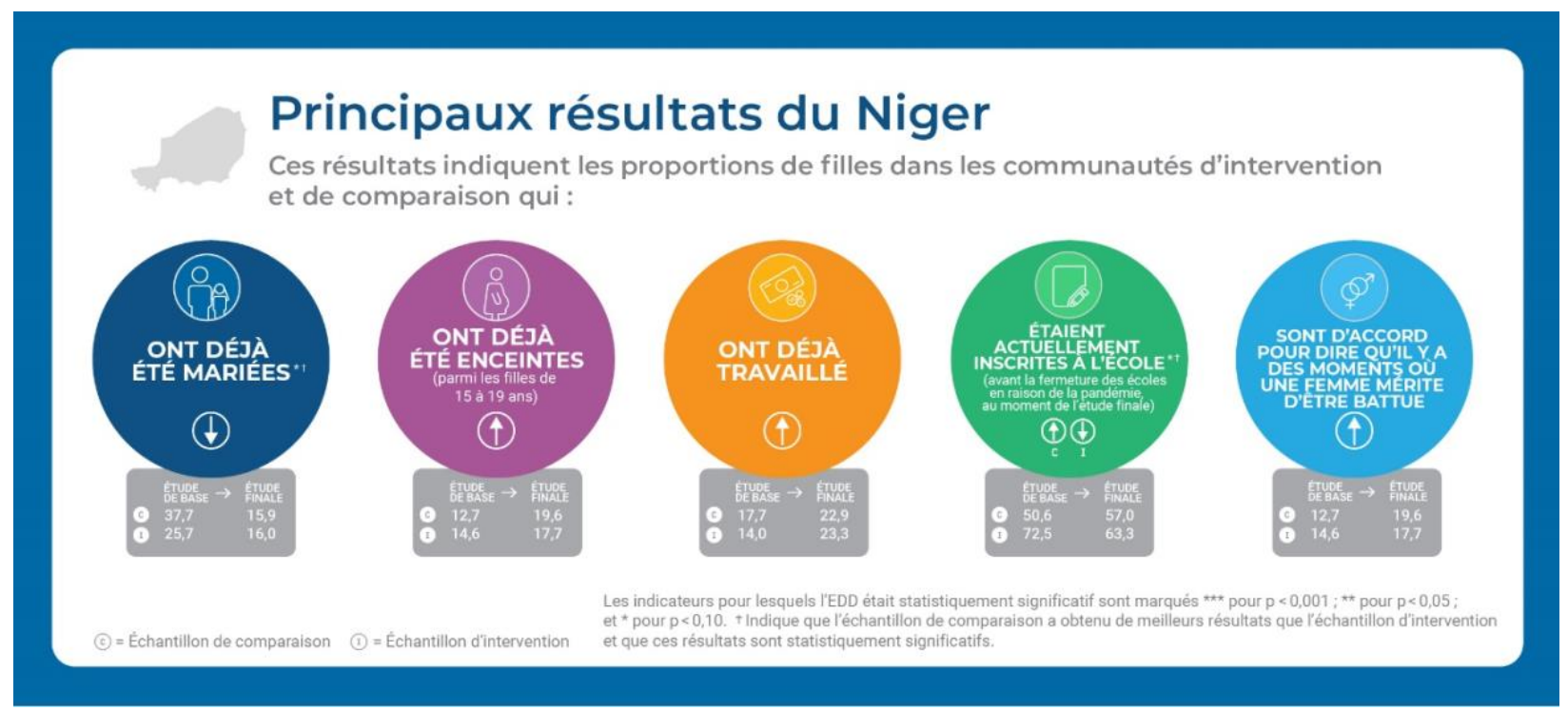

Les principales résultats sur des indicateurs clés comparés entre l'étude base et l'étude finale sont présentées dans la Figure 14. Le Tableau 13 ci-dessous présente les résultats de l'évaluation d'impact pour le Niger. Les flèches apparaissant à côté de INT et COM montrent la direction du changement pour chaque indicateur entre l'étude de base et l'étude finale dans les zones d'intervention et de comparaison, respectivement. Les indicateurs surlignés en bleu représentent l'impact du programme (EDD significatifs avec des zones d'intervention plus performantes que les zones de comparaison). Les indicateurs surlignés en or représentent les résultats pour lesquels l'EDD était significatif mais les zones de comparaison ont dépassé les zones d'intervention.

Table 13. Résultats de l'étude finale, Niger

\begin{tabular}{|c|c|c|}
\hline Domaine d'impact & Indicateur & $\begin{array}{l}\text { Direction du changement de } \\
\text { l'étude de base à l'étude finale }\end{array}$ \\
\hline \multirow{4}{*}{ Mariage } & Filles ayant déjà été mariées & INT $\downarrow$ COM $\downarrow$ \\
\hline & Filles pouvant identifier correctement l'âge légal du mariage & INT $\uparrow \mathrm{COM} \downarrow$ \\
\hline & $\begin{array}{l}\text { Filles pouvant identifier au moins trois conséquences néfastes liées } \\
\text { au mariage précoce }\end{array}$ & INT $\uparrow \mathrm{COM}-$ \\
\hline & Âge moyen au mariage & INT $\uparrow \mathrm{COM}-$ \\
\hline \multirow{5}{*}{ Santé } & Filles ayant déjà eu une grossesse (parmi les filles de 15 à 19 ans) & INT - COM - \\
\hline & Filles ayant des connaissances sur le VIH & INT $\uparrow \mathrm{COM} \mathrm{-}$ \\
\hline & Filles sachant que I'utilisation d'un préservatif protège du VIH & INT $\uparrow \mathrm{COM} \uparrow$ \\
\hline & $\begin{array}{l}\text { Filles indiquant que leur communauté dispose d'un centre de santé } \\
\text { adapté aux jeunes }\end{array}$ & INT $\uparrow \mathrm{COM} \uparrow$ \\
\hline & Échelle de connaissance des contraceptifs (méthodes modernes) & INT $\uparrow \mathrm{COM} \uparrow$ \\
\hline \multirow{2}{*}{$\begin{array}{l}\text { Moyens de } \\
\text { subsistance }\end{array}$} & Filles ayant déjà travaillé pour un revenu & INT $\uparrow \mathrm{COM}-$ \\
\hline & Filles travaillant actuellement pour un revenu & INT $\uparrow \mathbf{C O M} \uparrow$ \\
\hline
\end{tabular}




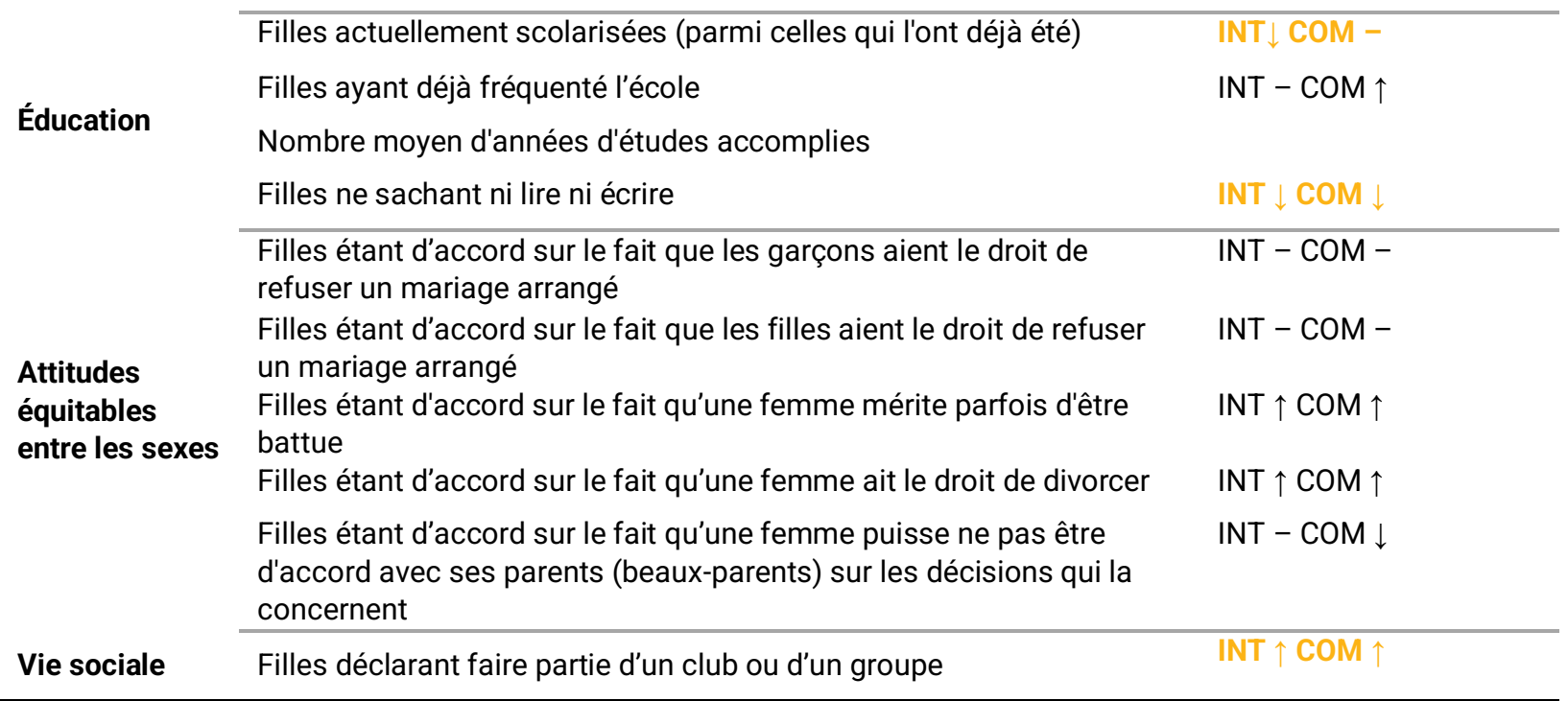

Note : Les résultats surlignés en bleu montrent les indicateurs pour lesquels l'EDD était significatif et les communautés d'intervention ont mieux performé que les communautés de comparaison. Les résultats surlignés en or montrent les indicateurs pour lesquels l'EDD était significatif et les communautés de comparaison ont mieux performé que les communautés d'intervention.

En examinant les effets néfastes du mariage des enfants au Niger, nous constatons que les filles des zones d'intervention ont obtenu des résultats significativement meilleurs que les filles des zones de comparaison, la proportion de filles pouvant citer au moins trois effets néfastes ayant presque doublé (de $15 \%$ à 29,6\%) et aucun changement significatif n'ayant été constaté dans les zones de comparaison. Nous notons également une augmentation de la proportion de filles pouvant identifier correctement l'âge légal du mariage pour les filles au Niger, qui est passé de $18,9 \%$ à $31,9 \%$ dans les communautés d'intervention avec un EDD significatif.

Nous notons que la proportion de filles déclarant avoir été mariées a diminué de manière significative dans les échantillons d'intervention et de comparaison avec un EDD significatif. Cependant, une diminution plus spectaculaire a été observée dans les villages de comparaison (de $37,7 \%$ à $15,9 \%$ ) par rapport aux villages d'intervention (25,7 \% à $16,0 \%$ ).

Une augmentation de l'âge moyen au mariage de 14,6 ans à 16,5 ans a été observée dans les zones d'intervention et aucun changement n'a été observé dans les zones de comparaison (l'âge moyen restant à 15,1 ans), cependant, l'EDD n'était pas significatif.

De l'étude de base à l'étude finale, nous avons constaté une augmentation de la connaissance des méthodes contraceptives modernes dans les villages d'intervention et de comparaison avec un EDD significatif, ce qui suggère que le programme MNCP a eu un impact sur ces indicateurs. La connaissance des méthodes contraceptives est passée de $48,3 \%$ à $64,4 \%$ dans les villages d'intervention et de $49,3 \%$ à $56,5 \%$ dans les villages de comparaison.

Dans les zones d'intervention et de comparaison, nous avons observé une augmentation de la proportion de filles sachant que l'utilisation d'un préservatif protège contre le VIH (de 18,3\% à $38,7 \%$ dans les zones d'intervention et de $18,3 \%$ à 27,9 \% dans les zones de comparaison) et de la proportion de filles ayant déclaré que leur communauté disposait d'un centre de santé adapté aux jeunes (de $2 \%$ à 17,9\% dans les villages d'intervention et de $2 \%$ à 15,6\% dans les villages de comparaison). 
La proportion de filles déclarant travailler actuellement pour obtenir un revenu a augmenté de manière significative dans les communautés d'intervention et de comparaison, avec un EDD significatif. Cette proportion a augmenté davantage dans les villages d'intervention (de $23,8 \%$ à $67,1 \%$ ) que dans les villages de comparaison ( $66 \%$ à $72,5 \%$ ), ce qui suggère que l'intervention du SRCM a pu jouer un rôle dans la mise en relation réussie des filles avec des opportunités de travail.

Si l'on considère les indicateurs d'éducation, la proportion de filles actuellement scolarisées a diminué dans les zones d'intervention (de $72,5 \%$ à $63,3 \%$ ) tout en restant relativement stable dans les zones de comparaison (augmentation de 50,6\% à $57 \%$; gain non significatif), ce qui représente une convergence entre les contextes. Les pertes dues à la pandémie de Covid-19 et à la fermeture des écoles qui en découlent peuvent avoir pour effet d'effacer les gains réalisés dans la scolarisation des filles dans les zones d'intervention depuis l'étude à mi-parcours. La proportion de filles ne sachant ni lire ni d'écrire a diminué dans les deux échantillons, mais moins dans les villages d'intervention (de 45 à $22,9 \%$ contre $64 \%$ à $31,9 \%$ dans les villages de comparaison).

En ce qui concerne les attitudes équitables entre les sexes, les tendances ne vont pas toujours dans le sens souhaité, reflétant peut-être des normes profondément ancrées autour du mariage ou la difficulté de traduire cette question dans le contexte nigérien. Bien que sur certains indicateurs, y compris la proportion de ceux qui sont d'accord avec le fait qu'une femme doit avoir le droit de divorcer, les filles des zones d'intervention ont montré des améliorations dans la direction souhaitée (de $22,3 \%$ à $35 \%$ ). Cependant, nous ne constatons aucun impact significatif par rapport aux zones de comparaison.

En phase d'étude finale, beaucoup plus de filles ont déclaré faire partie d'un club ou d'un groupe dans les zones de comparaison et d'intervention. Si les zones de comparaison ont obtenu de meilleurs résultats (de $28 \%$ à $79 \%$ ), les zones d'intervention ont également enregistré une forte augmentation (de $21 \%$ à $86 \%$ ). 


\section{Discussion}

\section{Les tendances du mariage : Étude de base-Étude à mi-parcours-Étude finale}

Tout au long de ce rapport, nous avons présenté les résultats de l'étude de base à l'étude finale par pays afin de démontrer comment le même programme semble avoir eu un impact sur différents indicateurs dans différents contextes au cours de la mise en œuvre du programme. Ici, nous examinons brièvement les tendances du mariage des enfants de l'étude de base à l'étude à mi-parcours et de l'étude à mi-parcours à l'étude finale. L'inclusion des données de l'étude à miparcours nous permet de voir si et comment les tendances de l'étude de base à la l'étude à miparcours ont pu se poursuivre, s'accélérer ou s'arrêter au cours des dernières années du programme. Les tendances entre l'étude de base, l'étude à mi-parcours et l'étude finale pour d'autres indicateurs clés sont fournies à l'annexe 3.

Figure 15. Tendances des filles déjà mariées, étude de base-étude à mi-parcours-étude finale

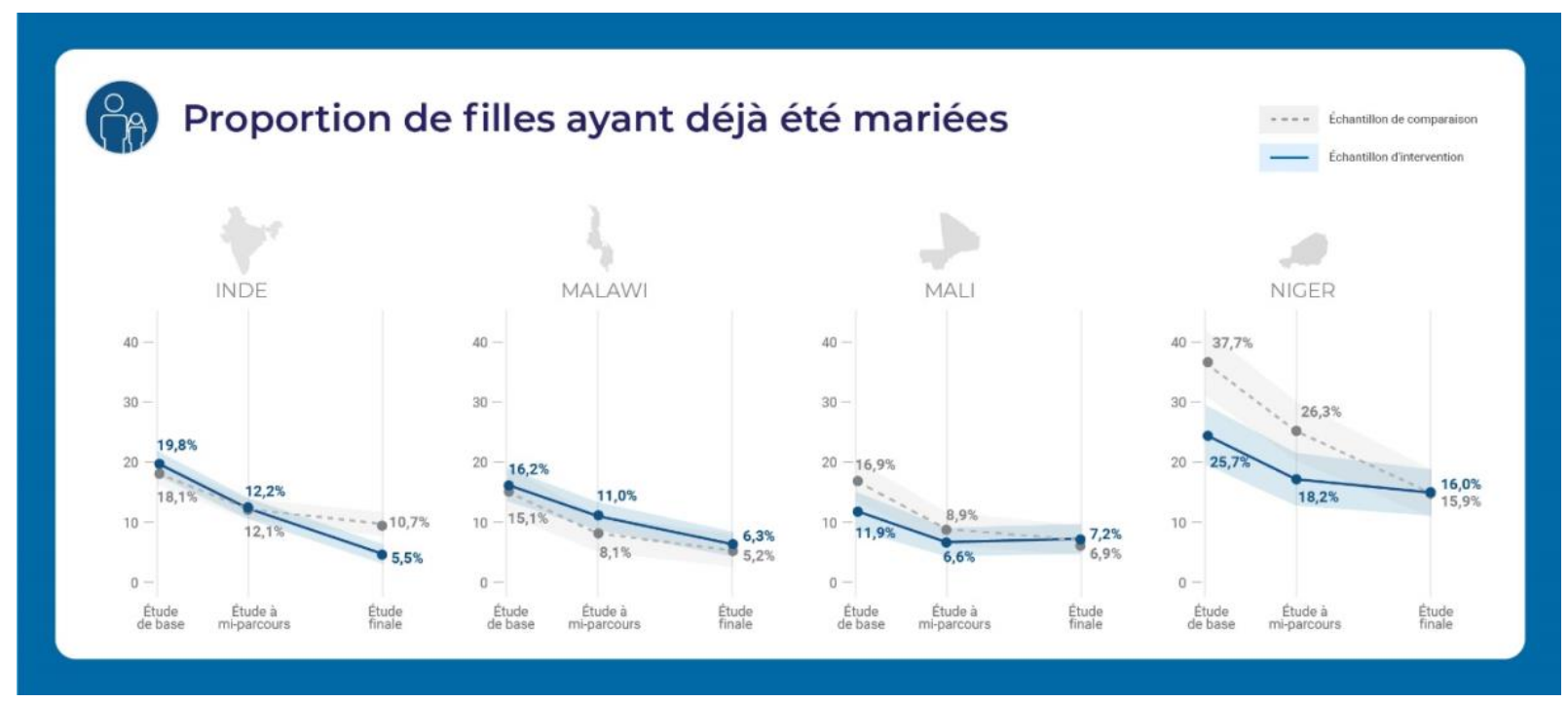

La Figure 15 montre les tendances de la prévalence du mariage des enfants dans les différents pays, y compris les points de données dans les échantillons d'intervention et de comparaison pour chaque cycle d'étude, avec les intervalles de confiance à $95 \%$ correspondants (représentés par des zones ombrées autour de chaque courbe de tendance). En Inde, la courbe entre l'étude de base et l'étude à mi-parcours est restée relativement constante entre l'étude à mi-parcours et l'étude finale dans les zones d'intervention, mais a légèrement diminué dans les zones de comparaison au cours des dernières années du programme. Au Malawi, les déclins précoces se sont poursuivis jusqu'à la fin du programme dans les zones d'intervention et de comparaison, tandis qu'au Mali, les augmentations obtenues entre l'étude de base et l'étude à mi-parcours semblent avoir stagné depuis l'étude à mi-parcours. Il est intéressant de noter qu'au Niger, dans les zones de comparaison, nous observons une forte baisse de la prévalence du mariage des 
enfants sur l'ensemble des trois phases temporelles, alors que dans les zones d'intervention, nous observons une forte baisse similaire de l'étude de base à l'étude à mi-parcours, mais ensuite une baisse plus lente depuis l'étude à mi-parcours, les deux groupes convergeant vers environ $16 \%$ des filles déclarant avoir été mariées à la fin du programme.

La prévalence du mariage a diminué dans tous les échantillons au cours de la période de mise en œuvre du programme MNCP, mais ce n'est qu'en Inde que le changement était attribuable à l'intervention (les baisses observées dans les zones d'intervention étaient significativement plus importantes que celles observées dans les zones de comparaison). Nous notons que le programme MNCP s'est déroulé à une époque où le mariage des enfants était en net recul au niveau mondial. Dans ce contexte, l'intensification des activités de plaidoyer aux niveaux mondial et national a probablement influencé le déclin réel du mariage des enfants et a peut-être aussi réduit le nombre de signalements, étant donné que la sensibilisation aux méfaits et/ou à l'illégalité de la pratique semble avoir augmenté au niveau communautaire au cours de cette période. 


\section{Impact du programme MNCP}

Le Tableau 14 ci-dessous montre les indicateurs sur lesquels le programme MNCP a démontré un impact significatif par rapport aux changements observés pendant la période d'intervention dans des villages similaires où le programme MNCP n'était pas présent.

Tableau 14. Résumé des résultats de l'impact

\begin{tabular}{|c|c|c|c|c|}
\hline & Inde & Malawi & Mali & Niger \\
\hline \multicolumn{5}{|l|}{ Mariage } \\
\hline Filles actuellement mariées & INT $\downarrow$ COM $\downarrow$ & & & \\
\hline Age moyen au mariage & & \multicolumn{3}{|c|}{ INT $\uparrow \mathrm{COM} \mathrm{-}$} \\
\hline $\begin{array}{l}\text { Filles pouvant identifier au moins trois } \\
\text { conséquences néfastes }\end{array}$ & INT $\uparrow \mathrm{COM} \uparrow$ & INT $\uparrow \mathrm{COM}-$ & & INT $\uparrow \mathrm{COM} \downarrow$ \\
\hline $\begin{array}{l}\text { Filles pouvant identifier au moins trois } \\
\text { conséquences néfastes }\end{array}$ & INT $\uparrow \operatorname{COM} \uparrow$ & & & INT $\uparrow \quad C O M-$ \\
\hline \multicolumn{5}{|l|}{ Santé } \\
\hline $\begin{array}{l}\text { Filles pouvant identifier au moins trois } \\
\text { conséquences néfastes }\end{array}$ & INT $\uparrow \operatorname{COM} \uparrow$ & & & \\
\hline
\end{tabular}

Échelle de connaissance des contraceptifs

INT $\uparrow \mathrm{COM} \uparrow$

(méthodes modernes)

\section{Moyens de subsistance}

Filles travaillant actuellement pour un revenu

INT $\uparrow \operatorname{COM} \uparrow$

\section{Éducation}

Filles ayant déjà fréquenté l'école

INT $\uparrow \mathrm{COM} \uparrow$

Filles actuellement scolarisées (parmi celles INT $\uparrow$ COM qui l'ont déjà été)

Nombre moyen d'années d'études INT $\uparrow \mathrm{COM} \mathrm{-}$

Filles ne sachant ni lire ni écrire $\quad$ INT $\downarrow$ COM $\downarrow$

\section{Vie sociale}

Filles déclarant faire partie d'un club ou d'un INT $\uparrow$ COM groupe 
Au-delà de la constatation que l'intervention en Inde a eu un impact sur la réduction de la prévalence du mariage des enfants (ou sur la prévention de l'augmentation du nombre de mariages d'enfants, comme on l'a vu dans les zones de comparaison de certains états indiens), un impact a été détecté sur quelques autres indicateurs liés à l'âge du mariage, à la connaissance du mariage des enfants et à la connaissance des SDSR. Au Mali, le programme a eu un impact sur l'augmentation de l'âge moyen au mariage pour les filles. En Inde, au Malawi, et au Niger, l'intervention du MNCP semble avoir joué un rôle dans l'augmentation de la proportion de filles capables d'identifier correctement l'âge légal du mariage. En Inde et au Niger, le programme a également permis de sensibiliser les filles aux effets néfastes du mariage des enfants au niveau communautaire. L'intervention du MNCP semble avoir augmenté la proportion de filles ayant des connaissances sur le VIH en Inde et la proportion de filles ayant des connaissances sur les contraceptifs modernes au Niger. Les résultats suggèrent donc que le programme MNCP a eu le plus fort impact sur les indicateurs liés aux connaissances. Le programme MNCP semble également être capable d'influencer les résultats comportementaux clés, bien que des questions subsistent quant à la raison pour laquelle nous ne constatons un impact que dans certains contextes.

Les résultats finaux révèlent un impact minimal sur les indicateurs liés aux moyens de subsistance, mais des résultats plus impressionnants en ce qui concerne l'éducation des filles. Au Malawi en particulier, l'intervention du MNCP a eu un impact démontrable sur plusieurs indicateurs liés à l'éducation, à savoir l'augmentation de la proportion de filles ayant déjà été scolarisées, l'augmentation du nombre moyen d'années d'études accomplies et la diminution de la prévalence de l'analphabétisme chez les filles de 12 à 19 ans. En Inde, le programme a eu une influence sur l'augmentation de la proportion de filles de la communauté inscrites à l'école. En Inde, le programme MNCP a également démontré son impact sur l'augmentation de la participation des filles à des groupes, clubs ou associations. Ces améliorations suggèrent que le programme MNCP a pu avoir un impact efficace sur l'engagement des filles et des parents en faveur de l'éducation des filles, malgré les difficultés de la scolarisation à distance engendrées par la pandémie de Covid-19.

Au Niger, nous constatons que le programme MNCP a eu un impact sur l'augmentation de la proportion de filles travaillant actuellement pour gagner leur vie. L'absence d'impact du programme sur la plupart des indicateurs des moyens de subsistance peut être en partie due à l'influence de la pandémie de Covid-19 et des restrictions associées, qui ont entraîné une contraction des économies locales et nationales et limité ultérieurement les possibilités de travailler pour les adolescentes. Les progrès en matière de scolarisation peuvent expliquer en partie l'absence d'amélioration des indicateurs de moyens de subsistance, car dans certaines régions, l'accent a été mis sur le maintien des filles à l'école plutôt que sur leur participation à des activités génératrices de revenus.

Malgré les efforts du programme MNCP pour changer les normes sociales qui perpétuent la pratique du mariage des enfants, nous n'avons trouvé aucun impact démontrable sur les indicateurs liés aux attitudes équitables entre les sexes. Nous reconnaissons la difficulté de changer des attitudes et des normes sur le rôle des filles et des femmes profondément ancrées dans des contextes où le mariage des enfants demeure une pratique courante. Par exemple, il peut être difficile pour les adolescentes d'accepter que les filles aient le droit de refuser un mariage arrangé si elles n'ont jamais vu cela se produire dans leur communauté. 


\section{Défis pour la démonstration de l'impact}

L'évaluation finale a été conçue pour produire des données comparables dans tous les contextes, en évaluant l'impact du programme par rapport aux groupes de comparaison. Le programme MNCP peut prétendre à un impact sur les indicateurs clés lorsque les changements constatés dans les zones d'intervention sont nettement supérieurs à ceux constatés dans les zones de comparaison. Nous constatons des tendances positives sur les résultats clés - y compris le mariage des enfants, l'éducation et la grossesse - dans la plupart des zones d'intervention. L'absence d'impact du programme détectée peut être due à un certain nombre de facteurs tels que les sources potentielles de contamination et les retombées, le placement du programme et les difficultés de mesure.

Le problème du mariage des enfants s'étant renforcé ces dernières années dans le secteur du développement, un nombre croissant de programmes ont été conçus afin d'éduquer les communautés sur les méfaits de cette pratique et travailler à son éradication, ce qui complique les efforts visant à mesurer les zones du programme par rapport aux communautés " témoins ". D'autres programmes de lutte contre le mariage des enfants peuvent avoir été actifs dans les zones de comparaison pendant la période d'intervention du MNCP, introduisant des sources potentielles de contamination. Nous savons également que les programmes multisectoriels comme le MNCP ont tendance à collaborer avec des acteurs de différents niveaux, ce qui peut avoir des répercussions dans les zones de comparaison. Par exemple, l'impact de l'activité de plaidoyer auprès des représentants du gouvernement national ou provincial ne se limite probablement pas à des villages ou zones d'intervention spécifiques.

De plus, le fait que le programme MNCP ait été placé dans des zones ayant bénéficié de précédentes interventions (comme au Mali et au Niger) plutôt que dans des zones sélectionnées de manière aléatoire (comme en Inde et au Malawi) peut avoir influencé par inadvertance les résultats qui favorisaient les zones de comparaison dans l'évaluation de l'impact. Par exemple, les zones de comparaison étaient relativement désavantagées dans l'étude de base en raison des difficultés d'appariement avec les zones d'intervention présélectionnées sur des facteurs tels que l'accès à une route principale et la présence d'une école dans la communauté (Annexe 2). Par conséquent, les améliorations dans les zones de comparaison entre l'étude de base et l'étude finale peuvent avoir compensé les gains réalisés dans les zones d'intervention dans l'analyse. À la Figure 15 vous pouvez voir clairement l'effet de la randomisation en observant la différence entre les échantillons d'intervention et de comparaison au niveau de l'étude de base en Inde et au Malawi, par rapport à la différence entre les deux échantillons au Mali et au Niger. Dans les pays où la sélection des villages d'intervention a été randomisée, la proportion de personnes mariées en phase d'étude de base se chevauchait dans tous les statuts de traitement.

Comme nous l'avons noté, le mariage des enfants est en déclin dans le monde entier et comme la pratique devient moins courante (ou moins souvent rapportée), il devient également plus difficile de détecter l'impact du programme. En effet, à mesure que l'incidence diminue, des échantillons de plus grande taille sont nécessaires pour détecter un impact significatif. II peut également devenir de plus en plus difficile de mesurer avec précision la prévalence du mariage des enfants, car la connaissance croissante des lois sur l'âge minimum du mariage et la reconnaissance grandissante des méfaits du mariage des enfants peuvent encourager la sousdéclaration. Les tendances et les modèles de migration peuvent également fausser les mesures de l'impact du programme au niveau communautaire car les filles ayant pu interagir avec le 
programme mais ayant ensuite migré hors de leur village ne sont pas prises en compte dans les communautés d'intervention en phase d'étude finale. Des recherches qualitatives suggèrent qu'au Mali, par exemple, la migration est courante chez les adolescentes (Hertrich \& Lesclingand, 2013 ; Engebretsen et al., 2020). Dans les régions du Mali caractérisées par des taux élevés de migration des adolescentes, les enquêtes transversales répétées sous-estiment la prévalence des filles scolarisées, participant à des activités génératrices de revenus ou mariées lorsqu'elles ont quitté leur village pour l'une de ces raisons. 


\section{Limites de l'étude}

L'étude finale du programme MNCP présente un certain nombre de limites à prendre en compte parallèlement aux résultats. Les perturbations liées à la pandémie de Covid-19 ont bouleversé les méthodes de travail habituelles dans le secteur et ont imposé des changements importants dans notre méthodologie de collecte de données. Pour l'étude de base et à mi-parcours, les données ont été recueillies par le biais du recensement des ménages et d'entretiens approfondis avec des jeunes filles âgées de 12 à 19 ans, chez elles ou dans un espace privé de leur choix. Cependant, afin de terminer l'évaluation de l'étude finale en 2020, les équipes responsables de la collecte des données ont dû s'adapter aux restrictions liées au contrôle de la pandémie ainsi qu'aux limites imposées pour les déplacements et la collecte de données en face à face, en passant aux enquêtes téléphoniques. Le contenu des 'enquêtes a été réparti sur plusieurs appels téléphoniques et des enquêtes supplémentaires auprès des parents des jeunes filles et d'autres membres adultes de leur famille ont été menées pour recueillir les informations normalement collectées lors de l'exercice de recensement des ménages. Par conséquent, les différences constatées entre les filles échantillonnées pour l'étude finale et les filles échantillonnées pour l'étude de base et à mi-parcours peuvent avoir influencé les résultats (voir les rapports d'évaluation de base et à mi-parcours du MNCP pour connaître les caractéristiques de chaque échantillon des cycles précédents de cette étude). Nous avons également suivi un calendrier de collecte de données abrégé, terminant la collecte et l'analyse des données au premier trimestre 2021, après l'affectation de nombreux membres du personnel impliqués dans la mise en œuvre du programme MNCP à d'autres postes. Cela a limité notre capacité à collaborer étroitement avec le personnel du programme pour interpréter les résultats, comme nous l'avions fait après l'évaluation de mi-parcours.

Nous notons que la possession et l'accès au téléphone ne sont pas répandus dans ces pays, et qu'ils sont particulièrement limités au Malawi et au Niger. Les filles ayant répondu aux enquêtes téléphoniques de l'étude finale peuvent être différentes des filles qui auraient été interrogées dans le cadre d'entretiens en face à face. Les taux élevés de migration dans certains contextes, notamment au Mali, ont posé des défis supplémentaires à notre méthodologie d'échantillonnage. Dans certaines régions, nous avons dû procéder à un sur-échantillonnage car de nombreuses adolescentes étaient parties du village pour se marier, étudier, travailler ou retrouver des membres de leur famille dans d'autres communautés. Étant donné que les adaptations mises en place en réponse à la pandémie de Covid-19 persisteront probablement dans des contextes de crise ou d'insécurité permanente, les prochaines enquêtes qui seront menées sur les adolescentes devront prévoir ces changements. II faudra remédier aux inégalités d'accès aux téléphones mobiles et aux problèmes de déplacements des adolescentes afin de maximiser la validité et la fiabilité des échantillons de l'étude et augmenter les chances que les évaluations permettent de détecter l'impact du programme au niveau communautaire.

\section{Implications pour les programmes futurs}

L'évaluation du programme MNCP nous offre l'opportunité d'examiner les effets d'un programme multisectoriel visant à réduire le mariage des enfants dans divers contextes. Le projet MNCP a été mis en œuvre au niveau communautaire selon des stratégies éprouvées (par exemple, des clubs de filles offrant une éducation sur la santé et les droits sexuels et reproductifs et mettant les filles en contact avec les services de santé et d'éducation) visant à créer un environnement favorable aux filles, notamment avec des interventions impliquant les parents, la communauté 
au sens large et les représentants du gouvernement. Ce projet a été mis en œuvre en Inde, où la prévalence du mariage des enfants a diminué au cours de la dernière décennie, et au Niger où cette pratique reste obstinément élevée. L'intervention a été mise en œuvre dans des contextes où les relations sexuelles avant le mariage sont rares et taboues (Niger et Inde) et où les relations sexuelles avant le mariage sont plus courantes (Mali et Malawi).

Malgré une approche similaire dans chaque contexte, les résultats sont inégaux, l'intervention ayant eu le plus d'impact en Inde. Les résultats de l'évaluation du programme MNCP remettent en question notre conception selon laquelle il est plus facile de démontrer l'efficacité dans les zones où le mariage des enfants est le plus élevé. En effet, nous constatons des améliorations significatives même à Odisha, en Inde, où la prévalence du mariage des enfants était de 8,9\% chez les filles âgées de 12 à 19 ans dans les zones d'intervention pour l'étude de base, alors que nous ne constatons aucune amélioration significative au Niger où la prévalence était de $25,7 \%$ pour l'étude de base. Nos résultats indiquent que même dans les régions où la prévalence est faible ou en déclin, les programmes de lutte contre le mariage des enfants peuvent avoir un impact, probablement parce que ces régions sont déjà sur la voie du changement.

Nos résultats suggèrent en outre la nécessité d'adapter davantage les programmes afin de tenir compte des facteurs contextuels contribuant au mariage des enfants, notamment les relations sexuelles et les grossesses avant le mariage, la réalité du mariage en tant que stratégie de subsistance pour les filles et les femmes, et le phénomène croissant de la migration des adolescentes. Dans les contextes où les interventions ont mis l'accent sur l'information et les services SDSR, mais où la grossesse prénuptiale reste un facteur important du mariage précoce, les programmes doivent mettre l'accent sur la création d'un environnement favorable en mesure de réduire le problème de la grossesse et du mariage précoce en tant que moyens d'assurer la subsistance de nombreuses filles. Les efforts déployés pour lutter contre le mariage des enfants qui ne prennent pas en compte de manière adéquate la pauvreté et les possibilités de moyens de subsistance alternatifs pour les filles risquent de rester lettre morte. En outre, nous prévoyons une augmentation de la migration des adolescentes au cours des prochaines années. Afin d'optimiser leur impact, les programmes mis en place dans les zones touchées doivent adapter leurs stratégies afin d'intervenir dans des communautés dynamiques avec des populations de plus en plus mobiles.

En mettant en œuvre des programmes multisectoriels dans des contextes très variés où le mariage des enfants est élevé, soit en termes de nombre absolu, soit en termes de prévalence, le programme MNCP apporte une contribution inestimable. Dans l'ensemble, nos données suggèrent que des programmes tels que le MNCP peuvent contribuer de manière significative à mettre fin à la pratique du mariage des enfants. Lorsque nous sommes en mesure d'identifier méthodologiquement l'impact des programmes au niveau communautaire (comme en Inde et, dans une moindre mesure, au Niger) nous constatons que l'approche spécifique adoptée par le MNCP, consistant à impliquer les communautés tout en se concentrant sur les filles, a eu un impact considérable sur les indicateurs de scolarisation et de santé des filles. En général, nous avons constaté des tendances positives au cours des quatre années pendant lesquelles nous avons observé des changements. Ces résultats sont prometteurs et devraient encourager la poursuite des efforts en vue d'apporter des changements positifs dans la vie des adolescentes de ces communautés. 


\section{Annexe 1 : Partenaires de la mise en œuvre par pays et état/ région}

\begin{tabular}{|c|c|c|c|c|}
\hline Country & State/Region & $\begin{array}{l}\text { Alliance } \\
\text { Partner(s) }\end{array}$ & Local Implementing Partners & $\begin{array}{l}\text { No. de } \\
\text { Villages }\end{array}$ \\
\hline \multirow{4}{*}{ Inde } & Jharkhand & Simavi & $\begin{array}{ll}- & \text { Network for Enterprise Enhancement et } \\
& \text { Development Support (NEEDS) } \\
- & \text { Child in Need Institute (CINI) }\end{array}$ & 279 \\
\hline & Bihar & $\begin{array}{l}\text { Simavi \& Save } \\
\text { The Children NL }\end{array}$ & $\begin{array}{ll}- & \text { Save the Children India } \\
- & \text { Bihar Voluntary Health Association (BVHA)* } \\
- & \text { Fakirana Sister Society (FSS)* } \\
- & \text { Samagra Seva Kendra (SSK) } \\
- & \text { Center for Health et Resource Management } \\
& \text { (CHARM) }\end{array}$ & 75 \\
\hline & Odisha & $\begin{array}{l}\text { Save the } \\
\text { Children NL }\end{array}$ & $\begin{aligned} \text { - } & \text { Save the Children India } \\
- & \text { Association for Social et Health Advancement } \\
& \text { (ASHA-ODISHA) } \\
- & \text { Social Welfare Agency et Training Institute (SWATI) } \\
- & \text { Voluntary Health Association of India (VHAI) }\end{aligned}$ & 210 \\
\hline & Rajasthan & $\begin{array}{l}\text { Save the } \\
\text { Children NL }\end{array}$ & $\begin{array}{l}\text { - } \quad \text { Save the Children India } \\
\text { - } \quad \text { Shiv Shiksha Samiti Ranoli (SSSR) } \\
\text { - } \quad \text { Urmul }\end{array}$ & 45 \\
\hline \multirow{2}{*}{ Pakistan** } & Punjab & Oxfam Novib & $\begin{array}{l}\text { - Oxfam Pakistan } \\
-\quad \text { Bedari }\end{array}$ & 40 \\
\hline & Sindh & Oxfam Pakistan & $\begin{array}{ll}\text { - } & \text { Oxfam Pakistan } \\
- & \text { International Rescue Committee (IRC) } \\
- & \text { Baanhn Beli }\end{array}$ & 40 \\
\hline \multirow{2}{*}{ Malawi ${ }^{\star \star \star}$} & Mangochi & Simavi & - Youth Network et Counselling (YONECO) & 108 \\
\hline & Nkhata Bay & Simavi & - Girls Empowerment Network (GENET) & 45 \\
\hline \multirow[b]{2}{*}{ Mali } & Segou & $\begin{array}{l}\text { Save the } \\
\text { Children NL }\end{array}$ & $\begin{array}{l}\text { - } \quad \text { Save the Children Mali } \\
\text { - } \quad \text { AGIR pour l'environnement et la qualité de vie }\end{array}$ & 30 \\
\hline & Sikasso & Oxfam Novib & $\begin{array}{l}- \text { Oxfam Mali } \\
- \text { WALE } \\
- \text { FAWE } \\
- \text { A Soro }\end{array}$ & 12 \\
\hline \multirow[t]{2}{*}{ Niger } & Maradi & $\begin{array}{l}\text { Save The } \\
\text { Children NL }\end{array}$ & $\begin{array}{l}\text { - } \text { Save the Children Niger } \\
-\quad \text { Association pour le Bien-Etre Familiale (ANBEF) } \\
-\quad \text { SongES } \\
-\quad \text { ASEC Mungane } \\
-\quad \text { ADD Fassali }\end{array}$ & 10 \\
\hline & Tillabéri & Oxfam Novib & $\begin{array}{ll}- & \text { Oxfam Niger } \\
- & \text { SOS Femmes et Enfants Victimes de Violence } \\
& \text { Familiale (SOS FEVVF) } \\
- & \text { Association pour le Bien-Etre Familiale (ANBEF) }\end{array}$ & 32 \\
\hline
\end{tabular}




\section{Annexe 2 : Différences de conception des études}

\section{Conception d'une étude transversale randomisée en grappes en Inde et au Malawi}

En Inde et au Malawi, le programme MNCP n'a pas été conçu sur une intervention existante (comme ce fut le cas au Mali et au Niger). Par conséquent, l'équipe de recherche a pu randomiser la mise en œuvre de l'intervention MNCP. Une conception transversale randomisée en grappes a été réalisée car l'intervention a lieu au niveau communautaire et comprend plusieurs zones d'intervention; cette conception permet de comparer le programme complet du MNCP dans les zones d'intervention sans aucune intervention dans les zones de comparaison. À la mi-2016, les grappes (gram panchayats en Inde ; villages chefs de groupe au Malawi) ont été assignées de manière aléatoire aux groupes d'intervention ou de comparaison, avec un nombre égal de grappes désignées dans chaque groupe. L'unité de recherche est la ZD. Nous avons associé les villages de tête de groupe aux ZD sur la base des points GPS fournis par le programme.

Au Malawi, le nombre de grappes a été influencé par la présence géographique des partenaires de la mise en œuvre et l'existence d'un programme sur les moyens de subsistance similaire dans l'une des zones d'étude prévues. À Mchinji, l'existence d'un vaste programme sur les moyens de subsistance a été considérée comme une menace trop importante pour notre capacité à évaluer l'impact et a donc été retiré de l'échantillon d'évaluation d'impact, bien que Mchinji demeure une zone d'intervention du MNCP. Malheureusement, des problèmes de contamination au Malawi ont fait que certaines grappes, assignées comme zones de comparaison au départ, ont bénéficié de l'intervention et qu'une grappe, prévue comme intervention, n'avait bénéficié d'aucune intervention à la mi-2018. En raison de ces problèmes et parce que l'ampleur de la contamination était plus importante que ce qu'il était possible d'absorber dans notre échantillon, nous présentons les résultats tels qu'ils ont été mis en œuvre au Malawi comme le seul ajustement dans l'analyse EDD.

En Inde, nous disposons d'un échantillon plus important que dans les autres pays et sommes donc en mesure de fournir des comparaisons au niveau des états. Nous avons assigné de manière aléatoire les gram panchayats (GP) comme zones d'intervention ou de comparaison et utilisé les PSU correspondantes comme unité de recherche. Bien que certaines activités se déroulent au niveau du district ou du bloc et qu'elles peuvent influencer les GP désignés comme zones de comparaison, toutes les activités se déroulant à des niveaux géographiques inférieurs ont été mises en œuvre uniquement dans les GP désignés comme zones d'intervention.

\section{Conception quasi-expérimental appariée au Mali et au Niger}

Au Mali et au Niger, le choix du site du MNCP a été déterminé par la décision des responsables de la mise en œuvre de s'appuyer sur un programme précédent, le programme My Rights My Voice (Mes droits, ma voix) mis en œuvre dans une vingtaine de communautés au Mali et dans 15 communautés au Niger, et qui s'est concentré sur la construction d'un soutien communautaire 
pour retarder le mariage. En raison de l'existence du projet My Rights My Voice et de l'expérience de la sélection et de la collaboration avec des partenaires locaux au Mali et au Niger, les partenaires du programme MNCP ont décidé de s'appuyer sur ces programmes et de mettre en œuvre l'intervention dans ces mêmes communautés. Par conséquent, une conception quasiexpérimentale appariée a été mise en œuvre pour cette étude transversale. Les communautés de comparaison ont été sélectionnées en fonction de leurs similarités avec les communautés qui avaient mis en œuvre le projet My Rights My Voice. Dans la mesure du possible, nous avons tenté de sélectionner des correspondances répondant aux critères suivants :

1. Peut avoir été sélectionnée comme communauté d'intervention du programme My Rights My Voice en fonction de l'accessibilité du village ;

2. Il y avait un nombre similaire d'écoles ;

3. Il y avait un nombre similaire de centres de santé ;

4. La taille de la population était similaire.

Comme au Mali et au Niger, le programme s'est appuyé sur des programmes déjà mis en œuvre dans certaines communautés, la mise en œuvre de l'initiative s'est limitée à ces zones, dont 50 villages au Mali et 42 villages au Niger. Par conséquent, la taille de l'échantillon était limitée. Nous n'avons pas pu attribuer l'intervention de manière aléatoire, et avons donc dû trouver des correspondances appropriées avec les villages d'intervention sélectionnés. Nos échantillons étaient limités à 40 grappes au Mali (20 interventions et 20 comparaisons) et à 30 grappes au Niger (15 interventions et 15 comparaisons). Les calculs de la taille des échantillons sont disponibles dans les rapports de référence à l'adresse https://morethanbrides.org. 


\section{Annexe 3 : Tendances des indicateurs clés : étude de base- étude à mi-parcours-étude finale}

Les figures suivantes présentent les tendances des indicateurs clés à travers les trois phases d'évaluation du programme MNCP en Inde, au Malawi, au Mali et au Niger. Les proportions sont présentées pour les échantillons d'intervention et les échantillons de comparaison, avec des intervalles de confiance à $95 \%$ représentés par les bandes ombrées autour de chaque courbe de tendance. Nous résumons ci-dessous les principales tendances pour chaque indicateur.

Proportion de filles de 15-19 ans ayant déjà été enceintes

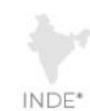

75

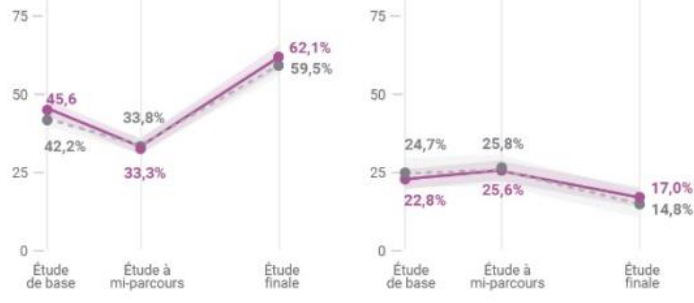

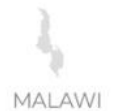

MALAWI

* Pour Ilinde, cette question n'a été posée qu'aux filles ayant déja été marièes.

En examinant la prévalence de la grossesse chez les adolescentes échantillonnées, on observe que les tendances se démarquent en Inde. Étant donné qu'en Inde, les questions relatives à la grossesse n'ont été posées qu'aux filles mariées âgées de 15 à 19 ans, les données présentées sur cet indicateur ne reflètent que les grossesses chez les filles mariées et ne sont pas comparables aux données présentées pour les trois autres pays. Dans les trois autres pays, nous constatons que la proportion de filles déclarant avoir déjà été enceintes est restée relativement stable pendant la période du programme. Au Niger, où les taux de mariage d'enfants sont les plus élevés et où l'initiation sexuelle a lieu presque exclusivement dans le cadre du mariage, la plupart des grossesses signalées concernent probablement des filles mariées. Au Malawi et au Mali, en revanche, la proportion de filles déclarant avoir déjà été enceintes est plus élevée que la proportion de filles déclarant avoir déjà été mariées à chaque phase de l'étude, ce qui suggère que dans ces pays des grossesses peuvent avoir lieu avant le mariage parmi les adolescentes. 


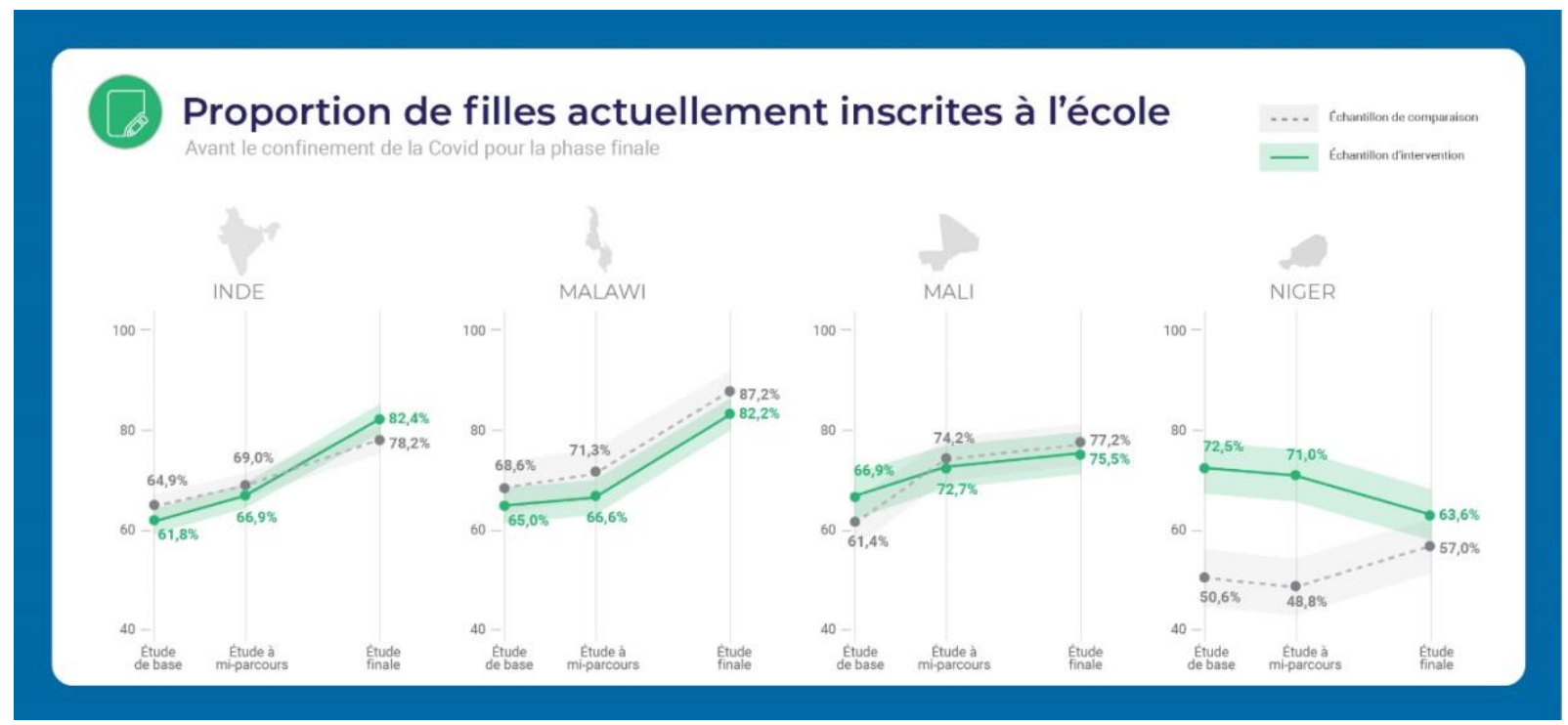

En ce qui concerne la scolarisation des filles, nous constatons des augmentations significatives dans les zones d'intervention et de comparaison dans tous les pays, à l'exception du Niger (notez que la question de l'étude finale portait sur la scolarisation juste avant la fermeture des écoles liées à la pandémie de Covid-19). Dans les zones d'intervention au Niger, la scolarisation des filles est demeurée relativement stable entre l'étude de base et l'étude à mi-parcours avant de diminuer sensiblement entre l'étude à mi-parcours et l'étude finale, alors que la tendance était opposée dans les communautés de comparaison. Cette tendance est probablement due en partie aux différences importantes entre les zones d'intervention et les zones de comparaison pour l'étude de base et aux défis de l'appariement avec les communautés d'intervention privilégiées.

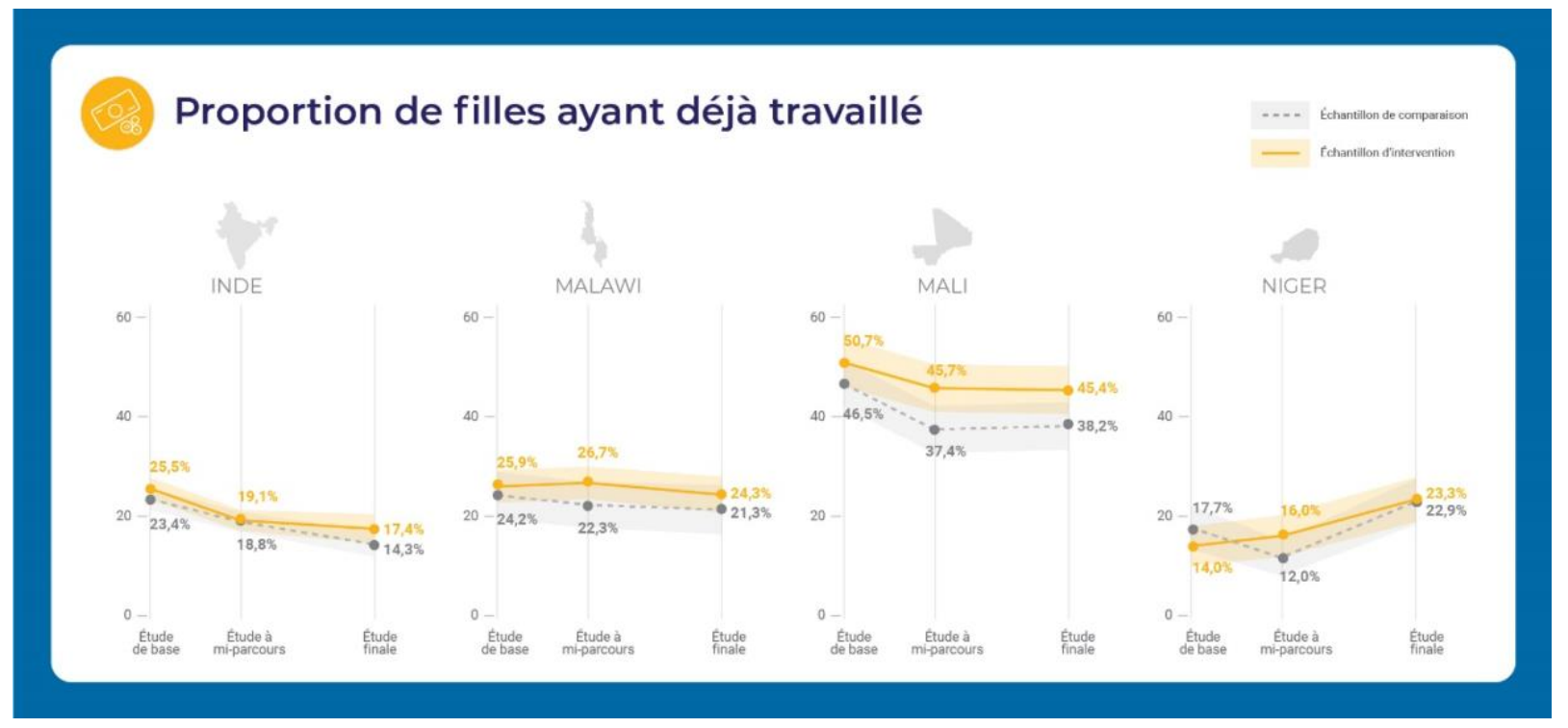

En examinant la proportion de filles ayant déclaré avoir déjà travaillé pour de l'argent, nous avons observé des baisses modérées dans la plupart des pays au cours de la période d'intervention, à l'exception du Niger, où cette proportion a régulièrement augmenté dans les communautés d'intervention. Cependant, malgré les augmentations observées au Niger, la proportion de filles ayant une expérience professionnelle reste la plus élevée au Mali (où nos données reflètent 
probablement une sous-estimation de la proportion réelle pour cet indicateur, en raison des taux élevés de migration liée au travail chez les adolescentes).

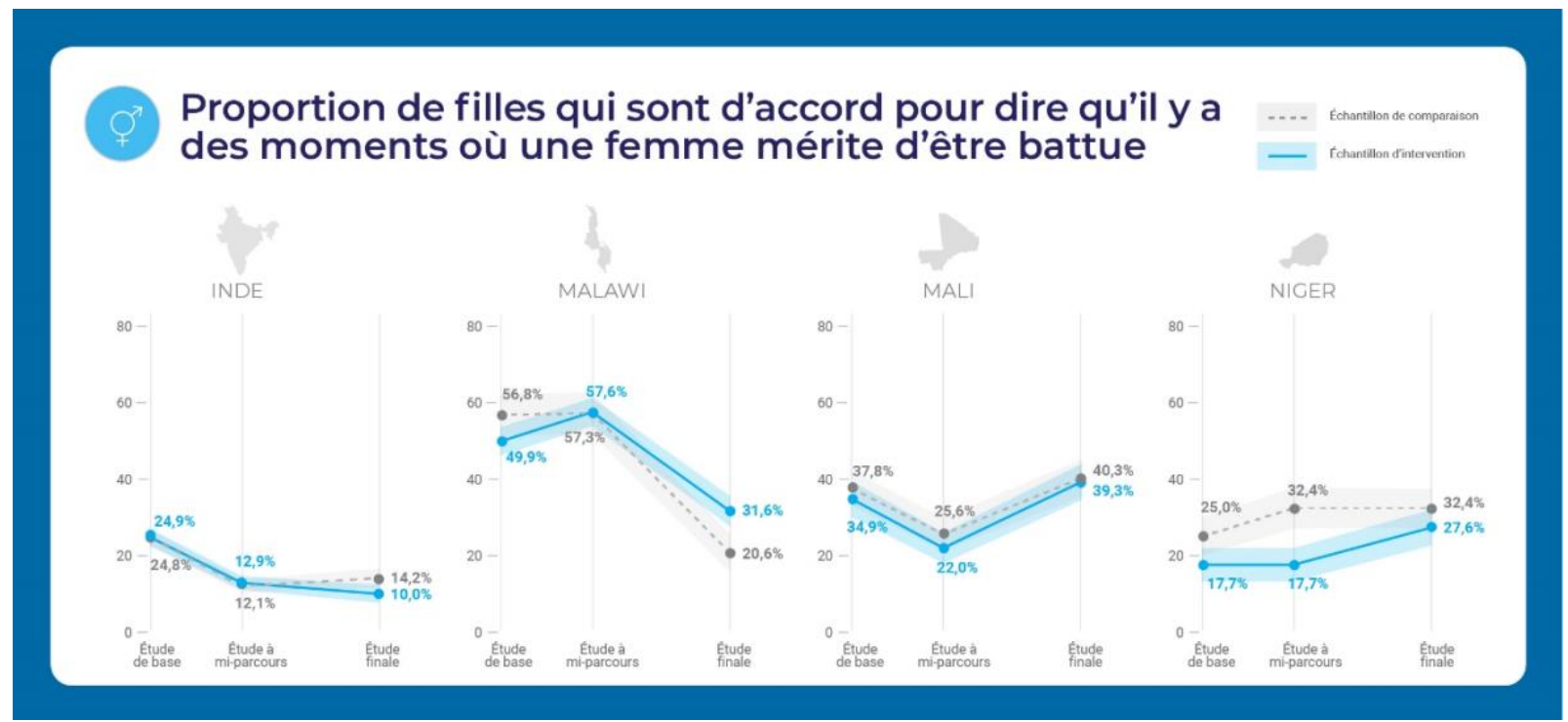

Nous constatons des progrès évidents en Inde et au Malawi en ce qui concerne l'acceptation de la violence, la proportion de filles étant d'accord sur le fait qu'il y a des moments où une femme mérite d'être battue ayant considérablement diminué entre l'étude de base et l'étude finale, dans les échantillons d'intervention et de comparaison. Au Mali, les nettes améliorations enregistrées à mi-parcours dans les communautés d'intervention ont ensuite connu un inversement de tendance, reflétant probablement la détresse et la tension visibles dans les données relatives à l'impact de la pandémie de Covid-19 (les filles maliennes ont connu une dépression plus élevée et une charge de travail majeure) ou aux changements dans l'échantillon dus à la migration et à la collecte de données mobiles. Au Niger, presque aucun changement n'a été observé dans les communautés d'intervention entre l'étude de base et l'étude à mi-parcours, mais des augmentations ont été observées entre l'étude à mi-parcours et l'étude finale. 


\section{Références}

Alston M, Whittenbury K, Haynes A, Godden N. 2014. "Are climate challenges reinforcing child and forced marriage and dowry as adaptation strategies in the context of Bangladesh?" Women Studies International Forum 47(A):137-144.

Amin S, Saha JS, Ahmed JA. 2018. "Skills-building programs to reduce child marriage in Bangladesh: A randomized controlled trial," J Adolesc Health 63 (3): 293-300.

Andriano L, \& Behrman J. 2020. "The effects of growing-season drought on young women's life course transitions in a sub-Saharan context," Population Studies 74(3): 331-350.

Ansell N, Hadju F, van Blerk L, Robson E. 2018. "'My happiest time' or 'my saddest time'? The spatial and generational construction of marriage among youth in rural Malawi and Lesotho," Trans Inst Br Geogr 43(2):184-199.

Baird S, McIntosh C, Ozler B. 2016. "When the Money Runs Out Do Cash Transfers Have Sustained Effects on Human Capital Accumulation?" World Bank Group Policy Research Working Paper 7901.

Bandiera O, Buehren N, Goldstein M, Rasul I, Smurra A. 2018. "The Economic Lives of Young Women in the Time of Ebola: Lessons from an Empowerment Program," Washington, SC: World Bank.

Biddlecom A, Gregory R, Lloyd CB. 2008. "Associations between Premarital Sex and Leaving School in Four Sub-Saharan African Countries," Studies in Family Planning 39(4): 337350.

Chae S, Ngo T. 2017. "The global state of evidence on interventions to prevent child marriage," GIRL Center Research Brief no.1. New York: Population Council.

Daniel EE, Nanda R. 2012. "The effect of reproductive health communication interventions on age at marriage and first birth in rural Bihar, India: a retrospective study." Watertown: Pathfinder International. Mastipur, Bodh Gaya, Bihar, India: Pathfinder International.

Demographic and Health Surveys. StatCompiler. Available at:

https://www.statcompiler.com/en/. Accessed March 9, 2021.

Engebretsen S, Gueye M, Melnikas AJ, Fofana S, Fané B, Amin S. 2020. "Adolescent girls' migration and its impact on early marriage: Qualitative findings in Mali," PLOS ONE 15(3): $1-18$.

Felton-Biermann C. 2006. "Gender and Natural Disaster: Sexualized violence and the tsunami', Development, 49(3): 82-86.

Fenn NS, Edmeades J, Lantos H Onovo O. 2015. "Child Marriage, Adolescent Pregnancy, and Family Formation in West and Central Africa," Washington DC and New York: ICRW and UNICEF. 
Gage A. 2013. "Association of Child Marriage With Suicidal Thoughts and Attempts Among Adolescent Girls in Ethiopia," Journal of Adolescent Health 52(5): 654-656.

Girls Not Brides. About child marriage. Girls Not Brides. Available at: https://www.girlsnotbrides.org/about-child-marriage/\#sources. Accessed March 10, 2021.

Glynn JR, Sunny BS, DeStavola B, Dube A, Chihana M, Price AJ, Crampin AC. 2018. "Early school failure predicts teenage pregnancy and marriage: A large population-based cohort study in northern Malawi," PLOS ONE 13(5): e0196041.

Greene M, Perlson S, Hart J. 2018. "The Centrality of Sexuality for Understanding Child, Early and Forced Marriage," Washington, DC and New York: American Jewish World Service

Grosz-Ngate M. 2000. "Labor Migration, Gender, and Social Transformation in Rural Mali," Democracy and Development in Mali, East Lansing: Michigan State University Press, 87101.

Hertrich V, Lesclingand M. 2013. "Adolescent migration in rural Africa as a challenge to gender and intergenerational relationships: Evidence from Mali," Ann Am Acad Pol Soc Sci 648(1): 175-188.

Hindin, M.J., Kishor S. Ansara, D.L. 2008. "Intimate partner violence among couples in 10 DHS countries: Predictors and health outcomes," DHS Analytical Studies No. 18. Calverton, Maryland, USA: Macro International Inc.

Hoogeveen J, van de Klaauw B, von Lomwell G.2011. "On the Timing of Marriage, Cattle, and Shocks," Economic Development and Cultural Change 60(1): 121-154.

Institut National de la Statistique (INS) et ICF International. 2013. "Enquête Démographique et de Santé et à Indicateurs Multiples du Niger 2012," Calverton, Maryland, USA: INS et ICF International.

Institut National de la Statistique (INSTAT), Cellule de Planification et de Statistique Secteur Santé-Développement Social et Promotion de la Famille (CPS/SS-DS-PF), et ICF. 2019. Enquete Démographique et de Santé au Mali 2018. Bamako, Mali, et Rockville, MD, USA: INSTAT, CPS/SS-DS-PF, et ICF.

International Institute for Population Sciences (IIPS) and ICF. 2017. "India National Family Health Survey (NFHS-4) 2015-16" Deonar, Mumbai, India: IIPS.

Jensen R. 2012. “Do labor market opportunities affect young women's work and family decisions? Experimental evidence from India," Q J Econ 127: 753-792.

Kalamar AM, Lee-Rife S, Hindin MJ. 2016. “Interventions to prevent child marriage among young people in low- and middle-income countries: A systematic review of the published and gray literature," J Adolesc Health 59 (3 Suppl):16-21.

Kelly CA, Melnikas AJ, Amin S, Mkandawire, Daud H. 2017. "More Than Brides Alliance: Baseline report, Malawi," New York: Population Council. 
Kim S. 2020. "Gender earnings gap among the youth in Malawi," African Development Review 32(2): 176-187.

Lee-Rife S, Malhotra A, Warner A, Glinski AM. 2012. "What works to prevent child marriage: A review of the evidence," Stud Fam Plann 43: 287-303.

Malhotra A, Elnakib S. 2021. "20 Years of the Evidence Base on What Works to Prevent Child Marriage: A Systematic Review," Journal of Adolescent Health: 1-16.

Melnikas AJ, Saul G, Singh S, Mkandawire J, Gueye M, Diarra A, Amin S. 2019. "More than Brides Alliance: Midline Evaluation Report." New York: Population Council.

Melnikas A, Ainul S, Ehsan I, Haque E, Amin S. 2020. "Child marriage practices among the Rohingya in Bangladesh," Conflict and Health 14(28).

Mensch, B., Bruce, J. and Green, M. 1998. “The uncharted passage: Girls' adolescence in the developing world," New <York: Population Council.

More Than Brides Alliance. 2016-2021: More Than Brides Alliance. Available at: http://morethanbrides.org.

Mourtada R, Schlect J, DeLong J. 2017. "A qualitative study exploring child marriage practices among Syrian conflict-affected populations in Lebanon," Conflict and Health 11(1):27.

National Statistical Office (NSO) [Malawi] and ICF. 2017. "Malawi Demographic and Health Survey 2015-16," Zomba, Malawi, and Rockville, MD: NSO and ICF.

Nour N. 2009. Child marriage: a silent health and human rights issue. Reviews in Obstetrics \& Gynecology. 2(1): 51-56.

Pande R, Kurz K, Walia S, MacQuarrie K, Jain S. 2006. "Improving the reproductive health of married and unmarried youth in India," ICRW.

Population Council. 2016-2020. Testing Effective Approaches to Ending Child Marriage. Available at: https://www.popcouncil.org/research/testing-effective-approaches-to-endchild-marriage.

Population Council. 2017. “More than Brides Alliance: Qualitative Findings from Malawi,” New York: Population Council.

Saul G, Diarra A, Melnikas A, Amin S. 2021. "Voice Without Choice? Investigating Adolescent Girls' Agency in Marital Decision-making in Niger," Progress in Development Studies 20(4): 270-281.

Sunny BS, Elze M, Chihana M, Gondwe L, Crampin AC, Munkhondya M, Kondowe, S, Glynn R. 2017. "Failing to progress or progressing to fail? Age-for-grade heterogeneity and grade repetition in primary schools in Karonga district, northern Malawi," International Journal of Educational Development 52: 68-80.

Steinhaus M, Hinson L, Rizzo AT. 2019. "Measuring Social Norms Related to Child Marriage Among Adult Decision-Makers of Young Girls in Phalombe and Thyolo, Malawi," Journal of Adolescent Health 64(4): S37-S44. 
United Nations Children's Fund. 2018. “Child Marriage: Latest trends and future prospects,” New York: UNICEF.

United Nations Population Fund. 2020. "Millions more cases of violence, child marriage, female genital mutilation, unintended pregnancy expected due to the COVID-19 pandemic" April 28, 2020. Accessed March 9 2021: https://www.unfpa.org/news/millions-more-casesviolence-child-marriage-female-genital-mutilation-unintended-pregnancies.

Wetheridge L, Antonowicz L. 2014. “Child Marriage in West Africa and Cameroon: A Desk Review," PLAN WARO.

Wodon, Q, Male C, Nayihouba A, Onagoruwa A, Savadogo A, Yedan A, Edmeades J, Kes A, John N, Murithi L, Steinhaus M and Petroni S. 2017. "Economic Impacts of Child Marriage: Global Synthesis Report," Washington, DC: The World Bank and International Center for Research on Women. 